REPRESENTATION THEORY

An Electronic Journal of the American Mathematical Society

Volume 10, Pages 399-434 (October 3, 2006)

S 1088-4165(06)00295-0

\title{
PROPAGATION DE PAIRES COUVRANTES DANS LES GROUPES SYMPLECTIQUES
}

\author{
CORINNE BLONDEL
}

\begin{abstract}
Let $\pi$ be a self-dual supercuspidal representation of $G L(N, F)$ and $\rho$ a supercuspidal representation of $\operatorname{Sp}(2 k, F)$, with $F$ a local nonarchimedean field of odd residual characteristic. Given a type, indeed a $S p(2 N+2 k, F)$ cover, for the inertial class $[G L(N, F) \times S p(2 k, F), \pi \otimes \rho]_{S p(2 N+2 k, F)}$ satisfying suitable hypotheses, we produce a type, indeed a $S p(2 t N+2 k, F)$-cover, for the inertial class $\left[G L(N, F)^{\times t} \times S p(2 k, F), \pi^{\otimes t} \otimes \rho\right]_{S p(2 t N+2 k, F)}$, for any positive integer $t$. We describe the corresponding Hecke algebra as a convolution algebra over an affine Weyl group of type $\tilde{C}_{t}$ with quadratic relations inherited from the case $t=1$ and the structural data for $\pi$.
\end{abstract}

Soit $F$ un corps local non archimédien de caractéristique résiduelle impaire et soit $G=S p(2 n, F)$ un groupe symplectique sur $F$. Le point de départ de ce travail est la question que m'a posée Guy Henniart il y a cinq ans sur la relation possible des travaux de Marko Tadić avec les paires couvrantes et leurs algèbres de Hecke. Voici de quoi il s'agit.

Fixons des entiers $N \geqslant 1$ et $k \geqslant 0$. Si $2 n=2 t N+2 k$ avec $t$ entier $\geq 1$, considérons dans $G=G_{t, k}$ le sous-groupe parabolique standard $P$ de facteur de Levi $M$ isomorphe à $G L(N, F) \times \cdots \times G L(N, F) \times S p(2 k, F)$ (avec $t$ copies de $G L(N, F)$ ). Soient $\pi$ et $\rho$ des représentations (complexes) irréductibles supercuspidales de $G L(N, F)$ et $S p(2 k, F)$ respectivement et regardons la représentation

$$
\pi \nu^{i_{1}} \times \cdots \times \pi \nu^{i_{t}} \rtimes \rho=\operatorname{Ind}_{P}^{G} \pi|\operatorname{det}|^{i_{1}} \otimes \cdots \otimes \pi|\operatorname{det}|^{i_{t}} \otimes \rho \quad\left(i_{1}, \cdots, i_{t} \in \mathbb{C}\right) .
$$

Les résultats de Tadić (voir en particulier [Ta] ) peuvent se traduire heuristiquement sous la forme suivante : les réductibilités et suites de composition des représentations ci-dessus sont déterminées par celles des représentations $\pi \nu^{i} \rtimes \rho(i \in \mathbb{C})$ dans $G_{1, k}=S p(2 N+2 k, F)$.

Comme $\pi \nu^{i} \rtimes \rho$, pour $i$ réel, est irréductible si $\pi$ n'est pas autoduale, je me limite dans la suite au cas d'une représentation $\pi$ autoduale. Supposons donnés des types $(\Gamma, \gamma)$ et $(\Delta, \delta)$ dans $G L(N, F)$ et $S p(2 k, F)$ attachés aux classes d'inertie respectives de $\pi$ et $\rho$, et supposons trouvée une paire couvrante $\left(\Omega_{1}, \omega_{1}\right)$ de $(\Gamma \times$ $\Delta, \gamma \otimes \delta)$ dans $G_{1, k}$ : c'est un type pour la classe d'inertie $[M, \pi \otimes \rho]_{G}$ dans $G_{1, k}$. La réductibilité de $\pi \nu^{i} \rtimes \rho(i \in \mathbb{C})$ et la structure d'une suite de composition sont alors entièrement déterminées par la structure de l'algèbre de Hecke associée $\mathcal{H}_{1}=\mathcal{H}\left(G_{1, k}, \omega_{1}\right)($ BK2 $\left.]\right)$.

Une version "types" des résultats de Tadić consisterait donc à établir qu'à partir de la paire $\left(\Omega_{1}, \omega_{1}\right)$ on peut construire une paire couvrante $\left(\Omega_{t}, \omega_{t}\right)$ de $(\Gamma \times \cdots \times \Gamma \times$

Received by the editors September 28, 2005.

2000 Mathematics Subject Classification. Primary 22E50; Secondary 20 C08.

(C)2006 American Mathematical Society Reverts to public domain 28 years from publication 
$\Delta, \gamma \otimes \cdots \otimes \gamma \otimes \delta)$ dans $G_{t, k}$ - donc un type pour la classe d'inertie $[M, \pi \otimes \cdots \otimes \pi \otimes \rho]_{G}$ dans $G_{t, k}$ - telle que l'algèbre de Hecke $\mathcal{H}_{t}=\mathcal{H}\left(G_{t, k}, \omega_{t}\right)$ correspondante soit entièrement déterminée par $\mathcal{H}_{1}$, i.e. définie par générateurs et relations se déduisant directement d'une présentation de $\mathcal{H}_{1}$.

C'est ce que nous établissons dans la suite, moyennant un choix judicieux du type $(\Gamma, \gamma)$ attaché à la représentation supercuspidale autoduale $\pi$ (on choisit un type simple maximal de Bushnell-Kutzko possédant les propriétés d'autodualité rappelées en 2.1.c, comme on peut le faire d'après [B13]) et à condition essentiellement que la trace sur $G_{1,0}$ de la paire couvrante $\left(\Omega_{1}, \omega_{1}\right)$ soit celle d'un type simple de Bushnell-Kutzko dans $G L(2 N, F)$.

En fait l'hypothèse exacte est à la fois plus et moins forte que cela. On part non pas d'une paire $\left(\Omega_{1}, \omega_{1}\right)$ mais de deux paires décomposées $(\Omega(1,1, k), \omega(1,1, k))$ et $(\Omega(1,2, k), \omega(1,2, k))$, au-dessus de $(\Gamma \times \Delta, \gamma \otimes \delta)$ dans $G_{1, k}$, "jumelles" : elles ne diffèrent que par leurs traces sur $G_{1,0}$ (plongé "aux quatre coins" de $G_{1, k}$ ) qui doivent être les intersections avec $G_{1,0}$ des sous-groupes $J_{P}$ et $J_{P^{-}}$de BushnellKutzko (paires couvrantes du type $(\Gamma \times \Gamma, \gamma \otimes \gamma)$ dans $G L(2 N, F)$ décrites dans BK1, §7). En contrepartie on ne demande pas tout à fait que ces paires soient couvrantes : il suffit que dans l'algèbre de Hecke de chacune d'elles un élément très particulier vérifie une relation quadratique de terme constant non nul. On trouvera les détails en 2.2. Noter qu'il peut arriver que les deux paires soient confondues, auquel cas les hypothèses faites entraînent immédiatement que la paire est couvrante; dans le cas général, elles sont effectivement couvrantes toutes deux par application du théorème 1.

A partir de ces ingrédients, le théorème 1 produit une famille de $t+1$ paires couvrantes $(\Omega(t, r, k), \omega(t, r, k)), t \leqslant r \leqslant 2 t$, de $(\Gamma \times \cdots \times \Gamma \times \Delta, \gamma \otimes \cdots \otimes \gamma \otimes \delta)$ dans $G_{t, k}$, dont les traces sur $G_{t, 0}$ sont les intersections avec $G_{t, 0}$ de paires couvrantes de Bushnell-Kutzko du type $\left(\Gamma^{\times 2 t}, \gamma^{\otimes 2 t}\right)$ dans $G L(2 t N, F)$. Les algèbres de Hecke $\mathcal{H}(t, r, k)=\mathcal{H}\left(G_{t, k}, \omega(t, r, k)\right)$ de ces paires sont deux à deux isomorphes pour $t \leqslant r \leqslant 2 t(2.7$, Proposition 5$)$ et contiennent une sous-algèbre remarquable $\mathcal{H}_{W}$, isomorphe à une algèbre de convolution sur un groupe de Coxeter de type $\tilde{C}_{t}$ et dont la présentation par générateurs et relations est entièrement déterminée par les coefficients des deux relations quadratiques de l'hypothèse (3.1, Proposition 6).

Pour montrer que $\mathcal{H}_{W}$ est égale à $\mathcal{H}(t, r, k)$, on doit faire une hypothèse supplémentaire : supposer que la représentation $\operatorname{Ind}_{\Delta}^{S p(2 k, F)} \delta$ est irréductible (donc isomorphe à $\rho$ dans le contexte ci-dessus). On peut alors utiliser la présentation de $\mathcal{H}(t, r, k)$ donnée par Bushnell et Kutzko, analogue à la présentation de Bernstein : voir (H4) et le théorème 2 en 3.3.

Disons maintenant un mot des deux lignes directrices de la démonstration.

La première est le pendant côté paires couvrantes de la "philosophie" sous-jacente aux travaux de Tadić mentionnés plus haut-voir l'introduction de [ST] : we have there more parabolic subgroups and we have more possibilities to compare informations coming from the Jacquet modules of various parabolic subgroups - plus le nombre de sous-groupes paraboliques pertinents est grand, plus le travail est facile. Une partie de la démonstration s'apparente en effet à un amusant "Rubik's cube" faisant apparaître des paires décomposées analogues, mais de taille inférieure, dans certains sous-groupes de Levi. Les propriétés des homomorphismes d'algèbres correspondants (définis par Bushnell et Kutzko, voir 1.1) remplacent alors celles des foncteurs de Jacquet. 
La seconde est l'introduction d'une famille de paires décomposées. La construction de cette famille repose sur une connaissance fine des types simples de Bushnell et Kutzko auxquels le paragraphe 1 est consacré : nous y précisons la forme, en termes de matrices par blocs, de ces types (Proposition 1), de façon à obtenir une famille de paires couvrantes dans $G L(t N, F)$ (Corollaire 1), dans laquelle les anneaux $\mathfrak{J}_{0}\left(\beta, \mathfrak{A}_{0}\right)$ et $\varpi_{E}^{-1} \mathfrak{H}_{1}\left(\beta, \mathfrak{A}_{0}\right)$ jouent des rôles concurrents (bien entendu il peut arriver que ces deux anneaux coïncident, la famille est alors réduite à un élément).

Une telle famille dans $G L(2 t N, F)$ est à la base de la construction au paragraphe 2 d'une famille de $t+1$ paires décomposées $(\Omega(t, r, k), \omega(t, r, k)), t \leqslant r \leqslant 2 t$, dans $G_{t, k}$ (moyennant les hypothèses déjà mentionnées pour $t=1$ ). Pour montrer que ces paires sont couvrantes, on se ramène à prouver que deux éléments particuliers, $E_{s(t, k)}$ et $E_{q(t, k)}$, de leur algèbre de Hecke sont inversibles. Or on sait obtenir l'inversibilité de $E_{s(t, k)}$ dans l'algèbre $\mathcal{H}(t, 2 t, k)$ et celle de $E_{q(t, k)}$ dans l'algèbre $\mathcal{H}(t, t, k)(2.6)$. Le point crucial est alors de produire des isomorphismes entre les algèbres $\mathcal{H}(t, r, k), t \leqslant r \leqslant 2 t$, permettant un bon contrôle du support (Proposition 5 de 2.7) ; ces isomorphismes sont aussi un outil majeur dans l'étude des algèbres $\mathcal{H}(t, r, k)$ faite au paragraphe 3.

La construction générale de paires couvrantes de $(\Gamma \times \Delta, \gamma \otimes \delta)$ dans $G_{1, k}$ est bien entendu une autre histoire, comme l'est celle du calcul effectif de leurs algèbres de Hecke, c'est-à-dire des paramètres des relations quadratiques vérifiées par les générateurs. Nous donnons cependant en 3.4 quelques exemples de paires satisfaisant les hypothèses voulues.

Cette étude doit beaucoup à Guy Henniart qui l'a suscitée et à Marko Tadić qui m'a longuement expliqué ses travaux. Je les en remercie, ainsi que Colette Mœglin pour des conversations très stimulantes.

\section{Les types simples de Bushnell et Kutzko}

Dans cette partie $F$ est un corps local non archimédien de caractéristique résiduelle $p$ quelconque, on note $\mathfrak{o}_{F}$ son anneau d'entiers, $\mathfrak{p}_{F}$ l'idéal maximal de $\mathfrak{o}_{F}$, $\varpi_{F}$ une uniformisante et $q_{F}$ le cardinal de son corps résiduel (notations analogues pour les extensions de $F$ ).

Le but est d'expliciter la forme d'un type simple de Bushnell et Kutzko en fonction du type simple maximal associé. Certaines définitions et notations nécessaires sont rappelées en 1.1 (paires couvrantes) et 1.2 (types simples dans $G L(N, F)$ ). La description cherchée des types simples est donnée en 1.3 (Proposition 1), ainsi que ses conséquences indispensables pour la deuxième partie. On obtient en particulier une famille de paires couvrantes (Corollaire 1) dont les algèbres de Hecke sont deux à deux isomorphes avec préservation du support, en un sens précisé en 1.4 (Proposition 2). La démonstration de la proposition essentielle (la proposition 1 de 1.3) est sans rapport avec le reste de l'article : on la trouvera en appendice.

1.1. Rappels sur les paires couvrantes. Commençons par rappeler rapidement les notions de paire décomposée et paire couvrante définies dans BK2].

Soit $G$ le groupe des points sur $F$ d'un groupe algébrique réductif et connexe défini sur $F$, soient $M$ un sous-groupe de Levi propre de $G$ et $P$ un sous-groupe parabolique de $G$ de facteur de Levi $M$ et radical unipotent $N$. On note $P^{-}$le sous-groupe parabolique de $G$ opposé de $P$ par rapport à $M$ et $N^{-}$son radical unipotent. 
On fixe un sous-groupe ouvert compact $J_{M}$ de $M$ et une représentation lisse irréductible $\tau_{M}$ de $J_{M}$ dans un espace vectoriel complexe $\mathcal{W}$.

On dit qu'un sous-groupe ouvert compact $J$ de $G$ porte une paire décomposée au-dessus de $\left(J_{M}, \tau_{M}\right)$ relativement à $P$ si :

(1) $J \cap M=J_{M}$ et $J=\left(J \cap N^{-}\right) J_{M}(J \cap N)$;

(2) il existe une représentation $\tau$ de $J$ dont la restriction à $J_{M}$ est égale à $\tau_{M}$ et dont les restrictions à $J \cap N^{-}$et $J \cap N$ sont triviales.

La représentation $\tau$ est alors entièrement déterminée et la paire $(J, \tau)$ est décomposée au-dessus de $\left(J_{M}, \tau_{M}\right)$ relativement à $P$.

Dans ces conditions, un élément $z$ de $M$ est dit positif relativement à $P$ et $J$, ou $(P, J)$-positif, si $z(J \cap N) z^{-1} \subset J \cap N$ et $z\left(J \cap N^{-}\right) z^{-1} \supset J \cap N^{-}$. Il est dit fortement positif s'il est positif et si de plus, pour tous sous-groupes ouverts compacts $H_{1}, H_{2}$ de $N$ (resp. $N^{-}$) il existe un entier $m$ positif (resp. négatif) tel que $z^{m} H_{1} z^{-m} \subset H_{2}$.

On dit que $J$ porte une paire couvrante de $\left(J_{M}, \tau_{M}\right)$ relativement à $P$ s'il porte une paire décomposée $(J, \tau)$ au-dessus de $\left(J_{M}, \tau_{M}\right)$ relativement à $P$ qui est une paire couvrante, i.e. qui vérifie :

(3) il existe un élément $z$ fortement positif du centre de $M$ tel que la double classe $J z J$ supporte un élément inversible de $\mathcal{H}(G, \tau)$.

Rappelons que l'algèbre de Hecke $\mathcal{H}(G, \tau)$ de $(J, \tau)$ est l'algèbre de convolution des fonctions lisses à support compact de $G$ dans $\operatorname{End}_{\mathbb{C}} \mathcal{W}$ vérifiant $f(k x h)=$ $\tau(k) f(x) \tau(h)$ pour tous éléments $h, k$ de $J$ et $x$ de $G$; elle est définie relativement à une mesure de Haar fixée sur $G$. On note $\mathcal{I}$ son élément unité. Son support est l'ensemble des éléments de $G$ qui entrelacent la représentation $\tau$, c'est-à-dire :

$$
I_{G}(\tau)=\left\{g \in G / I_{g}(\tau) \neq\{0\}\right\} \text {, avec } I_{g}(\tau)=\operatorname{Hom}_{J \cap J^{g}}\left(\tau, \tau^{g}\right) .
$$

On se permettra l'abus de notation suivant : pour tout $g \in G$ tel que le sousespace des éléments de $\mathcal{H}(G, \tau)$ de support contenu dans la double classe $J g J$ soit de dimension 1, on notera $E_{g}$ un élément non nul arbitraire de ce sous-espace. Un tel élément n'est déterminé qu'à un scalaire près, mais la plupart du temps cela nous suffira. La notation $f \equiv E_{g}$ signifiera que $f \in \mathcal{H}(G, \tau)$ est un multiple non nul de $E_{g}$.

Les résultats suivants ([BK2 Proposition 6.3, Corollaire 6.12 et Théorème 7.2) joueront un rôle essentiel dans la suite :

Théorème (Bushnell-Kutzko). Soit $(J, \tau)$ une paire décomposée au-dessus de $\left(J_{M}, \tau_{M}\right)$ relativement à $P$. On désigne par $M^{+}$l'ensemble des éléments $(P, J)$ positifs de $M$ et par $\mathcal{H}\left(M, \tau_{M}\right)^{+}$la sous-algèbre de $\mathcal{H}\left(M, \tau_{M}\right)$ formée des éléments de support contenu dans $M^{+}$. Soit c le quotient des volumes de $J$ et $J_{M}$ relativement à des mesures de Haar fixées sur $G$ et $M$ respectivement.

(i) On définit une injection d'espaces vectoriels $\mathcal{T}_{M}: \mathcal{H}\left(M, \tau_{M}\right) \underset{\text { ev }}{\hookrightarrow} \mathcal{H}(G, \tau)$ en associant à $f \in \mathcal{H}\left(M, \tau_{M}\right)$ de support $J_{M} m J_{M}$ la fonction $\mathcal{T}_{M}(f) \in \mathcal{H}(G, \tau)$ de support JmJ et valeur en $m: \mathcal{T}_{M}(f)(m)=c f(m)$. L'image de $\mathcal{T}_{M}$ est le sous-espace vectoriel des fonctions de support contenu dans JMJ.

(ii) On obtient par restriction de $\mathcal{T}_{M}$ un homomorphisme injectif d'algèbres à unité

$$
\mathcal{T}_{M}^{+}: \mathcal{H}\left(M, \tau_{M}\right)^{+} \underset{\mathrm{alg}}{\hookrightarrow} \mathcal{H}(G, \tau)
$$

dont l'image est la sous-algèbre des fonctions de support contenu dans $\mathrm{JM}^{+} J$. 
(iii) Si $(J, \tau)$ est une paire couvrante de $\left(J_{M}, \tau_{M}\right)$ relativement à $P$, l'homomorphisme $\mathcal{T}_{M}^{+}$se prolonge de manière unique en un homomorphisme injectif d'algèbres à unité

$$
\mathbb{T}_{M}^{+}: \mathcal{H}\left(M, \tau_{M}\right) \underset{\text { alg }}{\hookrightarrow} \mathcal{H}(G, \tau)
$$

Remarque. La définition d'une paire couvrante donnée ci-dessus est un peu plus faible que celle de Bushnell et Kutzko, qui supposent la paire décomposée par rapport à tout sous-groupe parabolique de facteur de Levi $M$. La plupart des résultats de BK2 que nous utiliserons restent valides avec cette définition plus faible (en particulier le théorème ci-dessus et le théorème de structure des algèbres de Hecke utilisé dans la troisième partie). Il nous faut en revanche préciser les propriétés de transitivité de [BK2] 8.5 qui restent valides (avec la démonstration de loc. cit.) dans notre situation :

Propriétés de transitivité (Bushnell-Kutzko). Soit $L$ un sous-groupe de Levi de $G$ contenu dans $M$ et $P^{\prime}$ un sous-groupe parabolique de $M$ de facteur de Levi $L$ et radical unipotent $N^{\prime}$. Soit $Q=P^{\prime} N$; c'est un sous-groupe parabolique de $G$ de facteur de Levi $L$ et radical unipotent $N^{\prime} N$. On note $J_{M}=J \cap M, \tau_{M}=\tau_{\mid J \cap M}$, $J_{L}=J \cap L$ et $\tau_{L}=\tau_{\mid J \cap L}$.

(i) Si $(J, \tau)$ est décomposée (resp. couvrante) au-dessus de $\left(J_{M}, \tau_{M}\right)$ relativement à $P$ et si $\left(J_{M}, \tau_{M}\right)$ est décomposée (resp. couvrante) au-dessus de $\left(J_{L}, \tau_{L}\right)$ relativement à $P^{\prime}$, alors $(J, \tau)$ est décomposée (resp. couvrante) au-dessus de $\left(J_{L}, \tau_{L}\right)$ relativement à $Q$.

(ii) Si $(J, \tau)$ est décomposée au-dessus de $\left(J_{L}, \tau_{L}\right)$ relativement à $Q$, alors la paire $\left(J_{M}, \tau_{M}\right)$ est décomposée au-dessus de $\left(J_{L}, \tau_{L}\right)$ relativement à $P^{\prime}$.

(iii) Si $(J, \tau)$ est décomposée (resp. couvrante) au-dessus de $\left(J_{L}, \tau_{L}\right)$ relativement $\grave{a} Q$ et si

$$
J \cap N^{\prime} N=\left(J \cap N^{\prime}\right)(J \cap N) \text { et } J \cap{N^{\prime-}}^{-}=\left(J \cap{N^{\prime}}^{-}\right)\left(J \cap N^{-}\right)
$$

alors $\left(J_{M}, \tau_{M}\right)$ est décomposée (resp. couvrante) au-dessus de $\left(J_{L}, \tau_{L}\right)$ relativement à $P^{\prime}$ et $(J, \tau)$ est décomposée (resp. couvrante) au-dessus de $\left(J_{M}, \tau_{M}\right)$ relativement $\grave{a} P$.

En pratique, nous travaillerons sur des sous-groupes $J$ de groupes linéaires ou symplectiques décrits sous forme de matrices par blocs par $J=\left(J_{i j}\right)$ où les $J_{i j}$ sont des réseaux si $i \neq j$ ou des sous-groupes ouverts compacts si $i=j$, autrement dit :

$$
J=\left\{x=\left(x_{i j}\right) \in G L\left(\sum_{i} n_{i}, F\right) \text { ou } S_{p}\left(\sum_{i} n_{i}, F\right) / x_{i j} \in J_{i j}\right\} .
$$

Soit $Q$ le sous-groupe des matrices triangulaires par blocs et $L$ le sous-groupe des matrices diagonales par blocs relativement à la décomposition en blocs ci-dessus, et supposons que $J$ porte une paire décomposée au-dessus de $L$ relativement à $Q$. Soit $M$ un sous-groupe de Levi standard dans cette situation, c'est-à-dire formé de blocs diagonaux de tailles des sommes $m_{s}=\sum_{i=i_{s}}^{i_{s+1}} n_{i}$. Alors la condition ( $\dagger$ ) ci-dessus est toujours vérifiée puisque :

$$
\left(\begin{array}{ccc}
g_{1} & & 0 \\
& \ddots & \\
0 & & g_{k}
\end{array}\right)\left(\begin{array}{ccc}
I_{m_{1}} & & \\
& \star & \\
& \ddots & \\
0 & & I_{m_{k}}
\end{array}\right)=\left(\begin{array}{ccc}
g_{1} & & \star^{\prime} \\
& \ddots & \\
0 & & g_{k}
\end{array}\right) .
$$

Notons d'ailleurs que le cas qui nous occupera relève aussi bien de la définition originale (voir le corollaire 3 de 2.4). 
1.2. Hypothèses et notations. On reprend le contexte de $\mathrm{Bl3}$ $\S 2.1$, cette fois avec $t$ blocs diagonaux au lieu de $2(t \geqslant 1$ un entier).

Soit $(\Gamma, \gamma)$ un type simple maximal dans $G L_{N}(F)$. D'après [BK1] (Définition 5.5.10, où l'on traite (b), dit cas de niveau 0 , comme un cas particulier de (a), et Théorèmes $6.2 .1,6.2 .2)$ la paire $(\Gamma, \gamma)=\left(J\left(\beta, \mathfrak{A}_{0}\right), \lambda\left(\beta, \mathfrak{A}_{0}\right)\right)$ est associée aux données suivantes :

(i) Un ordre principal $\mathfrak{A}_{0}$ sur $\mathfrak{o}_{F}$ et une strate simple $\left[\mathfrak{A}_{0}, n_{0}, 0, \beta\right]$ dans $M_{N}(F)$; en particulier $E=F[\beta]$ est une extension de $F$.

Soient $\mathfrak{P}_{0}$ le radical de $\mathfrak{A}_{0}$ et $B_{0}$ le commutant de $E$ dans $M_{N}(F)$. Alors $\mathfrak{B}_{0}=$ $\mathfrak{A}_{0} \cap B_{0}$ est un ordre maximal sur $\mathfrak{o}_{E}$ de radical $\mathfrak{Q}_{0}=\mathfrak{P}_{0} \cap B_{0}$.

(ii) Un caractère simple $\theta_{0} \in \mathcal{C}\left(\mathfrak{A}_{0}, 0, \beta\right)$ et une $\beta$-extension $\kappa_{0}$ à $J\left(\beta, \mathfrak{A}_{0}\right)$ de l'unique représentation irréductible $\eta_{0}$ de $J^{1}\left(\beta, \mathfrak{A}_{0}\right)$ contenant $\theta_{0}$.

(iii) Une représentation irréductible cuspidale $\sigma_{0}$ de $G L\left(f, k_{E}\right)$ relevée à $\Gamma$ via :

$$
J\left(\beta, \mathfrak{A}_{0}\right) / J^{1}\left(\beta, \mathfrak{A}_{0}\right) \simeq \mathbf{U}\left(\mathfrak{B}_{0}\right) / \mathbf{U}^{1}\left(\mathfrak{B}_{0}\right) \simeq G L\left(f, k_{E}\right), f=N /[E: F] .
$$

On a alors $\lambda=\kappa_{0} \otimes \sigma_{0}$.

Ecrivons $M_{t N}(F)$ sous la forme de matrices $t \times t$ dont les entrées appartiennent à $M_{N}(F)$. Cela correspond à une décomposition de l'espace vectoriel sous-jacent $V=$ $F^{t N}$, vu comme espace de matrices colonnes, en somme directe $V=\bigoplus_{j=1}^{t} V^{(j)}$ où $V^{(j)}$ est le sous-espace des matrices colonnes dont les seules entrées éventuellement non nulles sont les entrées $(t-j) N+1$ à $(t-j+1) N$.

Soit $\left(L_{0, i}\right)_{i \in \mathbb{Z}}$ la chaîne de réseaux de $F^{N}$ associée à l'ordre $\mathfrak{A}_{0}$. Elle détermine des chaînes de réseaux $\left(L_{0, i}^{(j)}\right)_{i \in \mathbb{Z}}$ dans $V^{(j)}, 1 \leqslant j \leqslant t$, moyennant l'identification naturelle de $V^{(j)}$ à $F^{N}$. Soit $\left(L_{i}\right)_{i \in \mathbb{Z}}$ la chaîne de réseaux de $V$ définie par :

$$
L_{t i+a}=\bigoplus_{j=1}^{a} L_{0, i+1}^{(j)} \oplus \bigoplus_{j=a+1}^{t} L_{0, i}^{(j)} \quad(i \in \mathbb{Z}, 0 \leqslant a<t) .
$$

Elle correspond à l'ordre principal $\mathfrak{A}=\mathfrak{A}_{t}=\left(\begin{array}{cccc}\mathfrak{A}_{0} & \mathfrak{A}_{0} & \ldots & \mathfrak{A}_{0} \\ \mathfrak{P}_{0} & \mathfrak{A}_{0} & \ddots & \vdots \\ \vdots & \ddots & \ddots & \mathfrak{A}_{0} \\ \mathfrak{\mathfrak { P }}_{0} & \cdots & \mathfrak{P}_{0} & \mathfrak{A}_{0}\end{array}\right)$ de $M_{t N}(F)$.

On identifie $E$ à son image diagonale par blocs dans $M_{t N}(F)$ : on écrit donc $\beta$ au lieu de $\left(\begin{array}{ccc}\beta & \cdots & 0 \\ \vdots & \ddots & \vdots \\ 0 & \ldots & \beta\end{array}\right)$. On note $B_{t}$ ou simplement $B$ le commutant de $E$ dans $M_{t N}(F)$ et on définit l'ordre sur $\mathfrak{o}_{E}: \mathfrak{B}=\mathfrak{B}_{t}=\mathfrak{A}_{t} \cap B_{t}$, de radical $\mathfrak{Q}=\mathfrak{P} \cap B$, où $\mathfrak{P}$ est le radical de $\mathfrak{A}$. La période de $\left(L_{i}\right)_{i \in \mathbb{Z}}$ est $t$ fois celle de $\left(L_{0, i}\right)_{i \in \mathbb{Z}} ;$ on a donc par BK1 1.2.11, 1.4.13, 1.2.4:

$\left[\mathfrak{A}, t n_{0}, 0, \beta\right]$ est une strate simple dans $M_{t N}(F)$ et toutes les hypothèses de [BK1] 7.1.11 et 7.2.1 sont vérifiées.

Notation. On raccourcit dans la suite les données relatives à $\left[\mathfrak{A}_{0}, n_{0}, 0, \beta\right]$ sous la forme $\mathfrak{H}^{1}=\mathfrak{H}^{1}\left(\beta, \mathfrak{A}_{0}\right), \mathfrak{J}^{1}=\mathfrak{J}^{1}\left(\beta, \mathfrak{A}_{0}\right), H^{1}=H^{1}\left(\beta, \mathfrak{A}_{0}\right), J^{1}=J^{1}\left(\beta, \mathfrak{A}_{0}\right)$ etc.

1.3. Description des types simples. Nous pouvons maintenant préciser, dans ce contexte, la structure des types simples de Bushnell et Kutzko dans $G L(t N, F)$ dont le type simple maximal associé est $(\Gamma, \gamma)$. 
Proposition 1. Les réseaux $\mathfrak{H}^{1}(\beta, \mathfrak{A})$ et $\mathfrak{J}^{1}(\beta, \mathfrak{A})$ de $M_{t N}(F)$ ont la forme suivante :

$$
\begin{aligned}
& \mathfrak{H}^{1}(\beta, \mathfrak{A})=\left(\begin{array}{ccccccc}
\longleftarrow & {\left[\frac{t}{2}\right]+1} & \longrightarrow & & & \\
\mathfrak{H}^{1} & \mathfrak{J}^{0} & \ldots & \mathfrak{J}^{0} & \varpi_{E}^{-1} \mathfrak{H}^{1} & \ldots & \varpi_{E}^{-1} \mathfrak{H}^{1} \\
\mathfrak{H}^{1} & \mathfrak{H}^{1} & \ddots & & \ddots & \ddots & \vdots \\
\vdots & \ddots & \ddots & & & \ddots & \varpi_{E}^{-1} \mathfrak{H}^{1} \\
\mathfrak{H}^{1} & & & & & & \mathfrak{J}^{0} \\
\varpi_{E} \mathfrak{J}^{0} & \ddots & & & \ddots & \ddots & \vdots \\
\vdots & \ddots & \ddots & & & \mathfrak{H}^{1} & \mathfrak{J}^{0} \\
\varpi_{E} \mathfrak{J}^{0} & \ldots & \varpi_{E} \mathfrak{J}^{0} & \mathfrak{H}^{1} & \ldots & \mathfrak{H}^{1} & \mathfrak{H}^{1}
\end{array}\right) \\
& \longleftarrow\left[\frac{t}{2}\right] \longrightarrow \\
& \mathfrak{J}^{1}(\beta, \mathfrak{A})=\left(\begin{array}{ccccccc}
\longleftarrow & {\left[\frac{t+1}{2}\right]} & \longrightarrow & & & \\
\mathfrak{J}^{1} & \mathfrak{J}^{0} & \ldots & \mathfrak{J}^{0} & \varpi_{E}^{-1} \mathfrak{H}^{1} & \ldots & \varpi_{E}^{-1} \mathfrak{H}^{1} \\
\mathfrak{H}^{1} & \mathfrak{J}^{1} & \ddots & & \ddots & \ddots & \vdots \\
\vdots & \ddots & \ddots & & & \ddots & \varpi_{E}^{-1} \mathfrak{H}^{1} \\
\mathfrak{H}^{1} & & & & & & \mathfrak{J}^{0} \\
\varpi_{E} \mathfrak{J}^{0} & \ddots & & & \ddots & \ddots & \vdots \\
\vdots & \ddots & \ddots & & & \mathfrak{J}^{1} & \mathfrak{J}^{0} \\
\varpi_{E} \mathfrak{J}^{0} & \ldots & \varpi_{E} \mathfrak{J}^{0} & \mathfrak{H}^{1} & \ldots & \mathfrak{H}^{1} & \mathfrak{J}^{1}
\end{array}\right) .
\end{aligned}
$$

Cette proposition est démontrée en appendice. Tirons-en tout de suite les conséquences. Soit $P$ le sous-groupe parabolique de $G L(t N, F)$ formé des matrices par blocs triangulaires supérieures, c'est-à-dire le fixateur du drapeau

$$
\{0\} \subset V^{(t)} \subset V^{(t)} \oplus V^{(t-1)} \subset \cdots \subset V^{(t)} \oplus \cdots \oplus V^{(2)} \subset V .
$$

On note $U$ son radical unipotent et $M$ le facteur de Levi des matrices diagonales par blocs, qui conservent chaque $V^{(j)}$. Soient enfin $P^{-}$l'opposé de $P$ par rapport à $M$ et $U^{-}$son radical unipotent. (Noter que $U$ est le $U^{-}$de [BK1] 7.1.13 et vice-versa.)

D'après BK1, $\$ 7.1$ et théorème 7.2 .17 , on a

$$
\begin{aligned}
J(\beta, \mathfrak{A})= & \left(J^{1}(\beta, \mathfrak{A}) \cap U^{-}\right) \cdot(J(\beta, \mathfrak{A}) \cap M) \cdot\left(J^{1}(\beta, \mathfrak{A}) \cap U\right) \\
& \text { avec } J(\beta, \mathfrak{A}) \cap M \simeq \Gamma \times \cdots \times \Gamma \quad(t \text { facteurs }) .
\end{aligned}
$$

En outre les groupes $J_{P}(\beta, \mathfrak{A})=\left(H^{1}(\beta, \mathfrak{A}) \cap U^{-}\right) \cdot(J(\beta, \mathfrak{A}) \cap M) \cdot\left(J^{1}(\beta, \mathfrak{A}) \cap U\right)$ et $J_{P^{-}}(\beta, \mathfrak{A})=\left(J^{1}(\beta, \mathfrak{A}) \cap U^{-}\right) .(J(\beta, \mathfrak{A}) \cap M) .\left(H^{1}(\beta, \mathfrak{A}) \cap U\right)$ portent chacun une paire couvrante de $(\Gamma \times \cdots \times \Gamma, \gamma \otimes \cdots \otimes \gamma)=\left(\Gamma^{\times t}, \gamma^{\otimes t}\right)$ relativement à $P$. Ces groupes sont aisés à décrire à l'aide de la proposition précédente :

- les blocs diagonaux sont égaux à $\Gamma$;

- les $\left[\frac{t-1}{2}\right]$ (pour $J_{P}$ ) ou $\left[\frac{t}{2}\right]$ (pour $J_{P^{-}}$) premières sous-diagonales au-dessus de la diagonale ont des blocs égaux à $\mathfrak{J}^{0}$; 
- les $\left[\frac{t-1}{2}\right]$ (pour $J_{P}$ ) ou $\left[\frac{t}{2}\right]$ (pour $J_{P^{-}}$) premières sous-diagonales au-dessous de la diagonale ont des blocs égaux à $\mathfrak{H}^{1}$;

- les autres sous-diagonales supérieures ont des blocs égaux à $\varpi_{E}^{-1} \mathfrak{H}^{1}$;

- les autres sous-diagonales inférieures ont des blocs égaux à $\varpi_{E} \mathfrak{J}^{0}$.

Par transitivité (voir 1.1), l'intersection d'un de ces groupes avec un sous-groupe de Levi de la forme $G L(k N, F) \times G L((t-k) N, F)$ plongé diagonalement par blocs, est encore une paire couvrante de $\left(\Gamma^{\times t}, \gamma^{\otimes t}\right)$ dans ce sous-groupe de Levi. En plongeant diagonalement $G L(t N, F)$ dans des $G L(i N, F)$ avec $i \geqslant t$ convenable, on obtient donc une famille de paires couvrantes dans $G L(t N, F)$ :

Corollaire 1. Pour tout entier $r$ tel que $\left[\frac{t+1}{2}\right] \leqslant r \leqslant t$, on définit :

$$
\Gamma(t, r)=\left(\begin{array}{ccccccc}
\longleftarrow & \mathfrak{J}^{0} & \ldots & \mathfrak{J}^{0} & \varpi_{E}^{-1} \mathfrak{H}^{1} & \ldots & \varpi_{E}^{-1} \mathfrak{H}^{1} \\
\mathfrak{H}^{1} & \Gamma & \ddots & & \ddots & \ddots & \vdots \\
\vdots & \ddots & \ddots & & & \ddots & \varpi_{E}^{-1} \mathfrak{H}^{1} \\
\mathfrak{H}^{1} & & & & & & \mathfrak{J}^{0} \\
\varpi_{E} \mathfrak{J}^{0} & \ddots & & & \ddots & \ddots & \vdots \\
\vdots & \ddots & \ddots & & & \Gamma & \mathfrak{J}^{0} \\
\varpi_{E} \mathfrak{J}^{0} & \ldots & \varpi_{E} \mathfrak{J}^{0} & \mathfrak{H}^{1} & \ldots & \mathfrak{H}^{1} & \Gamma
\end{array}\right) .
$$

Alors $\Gamma(t, r)$ est un sous-groupe de $G L(t N, F)$ qui porte une paire couvrante $(\Gamma(t, r), \gamma(t, r))$ de $\left(\Gamma^{\times t}, \gamma^{\otimes t}\right)$ relativement à $P$.

Remarque. Pour $r=t$ on retrouve la forme de Waldspurger Wa : les blocs audessus (resp. au-dessous) de la diagonale appartiennent tous au même réseau. Pour $r=\left[\frac{t+1}{2}\right]$ on retrouve bien entendu le groupe $J_{P}(\beta, \mathfrak{A})$ de Bushnell et Kutzko.

On peut affiner un peu ce résultat. D'après BK1 3.4.1, le groupe de commutateurs $\left[J^{1}(\beta, \mathfrak{A}), H^{1}(\beta, \mathfrak{A})\right]$ est contenu dans le noyau du caractère simple $\theta$ de $H^{1}(\beta, \mathfrak{A})$. Via les arguments ci-dessus, on obtient donc aussi :

Corollaire 2. Soient $H^{1}(t, r)$ et $J^{1}(t, r)$ les groupes obtenus en remplaçant, dans les blocs diagonaux de $\Gamma(t, r)$, le sous-groupe $\Gamma$ par $H^{1}\left(\beta, \mathfrak{A}_{0}\right)$ ou $J^{1}\left(\beta, \mathfrak{A}_{0}\right)$ respectivement. Le groupe $H^{1}(t, r)$ porte une paire décomposée $\left(H^{1}(t, r), \theta_{0}(t, r)\right)$ audessus de $\left(\left[H^{1}\right]^{\times t}, \theta_{0}{ }^{\otimes t}\right)$. De même, le groupe $J^{1}(t, r)$ porte une paire décomposée $\left(J^{1}(t, r), \eta_{0}(t, r)\right)$ au-dessus de $\left(\left[J^{1}\right]^{\times t}, \eta_{0}{ }^{\otimes t}\right)$. Enfin, $\Gamma(t, r)$ porte également une paire décomposée $\left(\Gamma(t, r), \kappa_{0}(t, r)\right)$ au-dessus de $\left(\Gamma^{\times t}, \kappa_{0}{ }^{\otimes t}\right)$.

Terminons par une propriété importante du réseau $\varpi_{E}^{-1} \mathfrak{H}^{1}$, qui contient $\mathfrak{J}^{0}$.

Lemme 1. Le réseau $\varpi_{E}^{-1} \mathfrak{H}^{1}$ est un sous-anneau de $M_{N}(F)$ dont les réseaux $\mathfrak{H}^{k}$ et $\mathfrak{J}^{k}$, pour $k \geqslant 1$, sont des idéaux bilatères ; en particulier : $\varpi_{E}^{-1} \mathfrak{H}^{1} \cdot \mathfrak{H}^{1}=\mathfrak{H}^{1}$.

Démonstration. Rappelons que $\mathfrak{H}^{1}=\mathfrak{H}^{1}\left(\beta, \mathfrak{A}_{0}\right)$. On montre par induction le long de $\beta$, comme dans [BK1] $\S 3$, que pour tout $k \geqslant 1$ :

$$
\mathfrak{H}^{1}(\beta, \mathfrak{A}) \mathfrak{H}^{k}(\beta, \mathfrak{A}) \subset \mathfrak{Q} \mathfrak{H}^{k}(\beta, \mathfrak{A}) \text { et } \mathfrak{H}^{1}(\beta, \mathfrak{A}) \mathfrak{J}^{k}(\beta, \mathfrak{A}) \subset \mathfrak{Q} \mathfrak{J}^{k}(\beta, \mathfrak{A}),
$$

et de même à droite. L'énoncé ci-dessus est l'application de cette propriété au cas où $\mathfrak{B}=\mathfrak{B}_{0}$ est maximal. 
1.4. Les algèbres de Hecke. On note $\mathcal{A}(t, r)$ l'algèbre de Hecke de la paire couvrante $(\Gamma(t, r), \gamma(t, r))$ ci-dessus, relativement à une mesure de Haar fixée sur $G L(t N, F)$ :

$$
\mathcal{A}(t, r)=\mathcal{H}(G L(t N, F), \gamma(t, r)) .
$$

Nous allons montrer que toutes ces algèbres (à $t$ fixé et $r$ variable) ont la même structure : celle de $\mathcal{A}\left(t,\left[\frac{t+1}{2}\right]\right)$, connue grâce à [BK1], 5.6.6 et 7.2.19. On introduit, comme dans loc. cit. $\S 5.5$, le groupe de Weyl affine étendu $\tilde{W}\left(\mathfrak{B}_{t}\right)$, produit semidirect de $D\left(\mathfrak{B}_{t}\right)=\left\{\operatorname{diag}\left(\varpi_{E}^{l_{1}} I_{N}, \cdots, \varpi_{E}^{l_{t}} I_{N}\right) / l_{1}, \cdots, l_{t} \in \mathbb{Z}\right\}$ et de $W_{0}\left(\mathfrak{B}_{t}\right) \simeq \mathfrak{S}_{t}$ formé des matrices de permutation par blocs dans $G L(t N, F)$.

Proposition 2. L'algèbre $\mathcal{A}(t, r)$ a pour support $\Gamma(t, r) \tilde{W}\left(\mathfrak{B}_{t}\right) \Gamma(t, r)$. Les doubles classes $\Gamma(t, r) w \Gamma(t, r)$ pour $w \in \tilde{W}\left(\mathfrak{B}_{t}\right)$ sont deux à deux disjointes et chacune supporte un sous-espace de dimension 1 de $\mathcal{A}(t, r)$ dont les éléments non nuls sont inversibles. L'algèbre $\mathcal{A}(t, r)$ peut être décrite par générateurs et relations comme dans BK1, 5.4.6.

Démonstration. La proposition est vraie pour $r=\left[\frac{t+1}{2}\right]$, il suffit donc de montrer que pour tout $r$ tel que $\left[\frac{t+1}{2}\right] \leqslant r<t$, il existe un isomorphisme d'algèbres de $\mathcal{A}(t, r)$ sur $\mathcal{A}(t, r+1)$ qui préserve le support au sens suivant :

l'image de $f \in \mathcal{A}(t, r)$ de support $\Gamma(t, r) w \Gamma(t, r), w \in \tilde{W}\left(\mathfrak{B}_{t}\right)$, a pour support $\Gamma(t, r+1) w \Gamma(t, r+1)$.

Les groupes $\Gamma(t, r)$ et $\Gamma(t, r+1)$ diffèrent par leurs intersections avec $U$ et $U^{-}$. On introduit le groupe $\Lambda(t, r)=\left(\Gamma(t, r+1) \cap U^{-}\right)(\Gamma(t, r) \cap M)(\Gamma(t, r) \cap U)$ qu'ils engendrent. Pour voir que ce produit est bien un groupe, on plonge $G L(t N, F)$ dans le sous-groupe de Levi standard $\mathcal{L}=G L(t N, F) \times G L((2 r-t) N, F)$ de $G L(2 r N, F)$. On vérifie qu'on a dans ce plongement :

$$
\begin{aligned}
\Lambda(t, r) & =J\left(\beta, \mathfrak{A}_{2 r}\right) \cap G L(t N, F), \\
\Gamma(t, r) & =J_{P}\left(\beta, \mathfrak{A}_{2 r}\right) \cap G L(t N, F), \\
\Gamma(t, r+1) & =J_{P^{-}}\left(\beta, \mathfrak{A}_{2 r}\right) \cap G L(t N, F) .
\end{aligned}
$$

En effet, la correspondance entre les notations du corollaire 1 ci-dessus et celles de BK1, $\S 7$, est donnée par $(\Gamma(2 r, r), \gamma(2 r, r))=\left(J_{P}\left(\beta, \mathfrak{A}_{2 r}\right), \lambda_{P}\left(\beta, \mathfrak{A}_{2 r}\right)\right)$ et $(\Gamma(2 r, r+$ $1), \gamma(2 r, r+1))=\left(J_{P^{-}}\left(\beta, \mathfrak{A}_{2 r}\right), \lambda_{P^{-}}\left(\beta, \mathfrak{A}_{2 r}\right)\right)$. Ce sont des paires décomposées par rapport à $(P, M)$ donc décomposées par rapport au parabolique standard de Levi $\mathcal{L}$. L'entrelacement dans $\mathcal{L}$ de la restriction de $\lambda_{P}\left(\beta, \mathfrak{A}_{2 r}\right)$ à $J_{P}\left(\beta, \mathfrak{A}_{2 r}\right) \cap \mathcal{L}$ est donc l'intersection avec $\mathcal{L}$ de l'entrelacement de $\lambda_{P}\left(\beta, \mathfrak{A}_{2 r}\right)$ dans $G L(2 r N, F)$ ([BK2] $6.3)$ et de même pour $\lambda_{P^{-}}\left(\beta, \mathfrak{A}_{2 r}\right)$. Or les représentations $\operatorname{Ind}_{J_{P}\left(\beta, \mathfrak{A}_{2 r}\right)}^{J\left(\mathfrak{A}_{2 r}\right)} \lambda_{P}\left(\beta, \mathfrak{A}_{2 r}\right)$ et $\operatorname{Ind}_{J_{P}\left(\beta, \mathfrak{A}_{2 r}\right)}^{J\left(\left(\mathfrak{A}_{2 r}\right)\right.} \lambda_{P^{-}}\left(\beta, \mathfrak{A}_{2 r}\right)$ sont irréductibles (loc. cit., 7.2.17). Un élément de $\Lambda(t, r)-\Gamma(t, r)$ ne peut donc pas entrelacer $\gamma(t, r)$, et de même pour $\gamma(t, r+1)$ : les représentations $\operatorname{Ind}_{\Gamma(t, r)}^{\Lambda(t, r)} \gamma(t, r)$ et $\operatorname{Ind}_{\Gamma(t, r+1)}^{\Lambda(t, r)} \gamma(t, r+1)$ sont irréductibles. Elles sont donc isomorphes, par réciprocité de Frobenius (on a $\Lambda(t, r)=\Gamma(t, r) \Gamma(t, r+1)$ ). Via BK1] 4.1.3, on obtient donc des isomorphismes d'algèbres :

$$
\begin{aligned}
\mathcal{A}(t, r) & \simeq \mathcal{H}\left(G L(t N, F), \operatorname{Ind}_{\Gamma(t, r)}^{\Lambda(t, r)} \gamma(t, r)\right) \\
& \simeq \mathcal{H}\left(G L(t N), \operatorname{Ind}_{\Gamma(t, r+1)}^{\Lambda(t, r)} \gamma(t, r+1)\right) \simeq \mathcal{A}(t, r+1) .
\end{aligned}
$$


L'assertion sur la préservation du support découle par récurrence sur $r$ de [BK1] 4.1.5, à condition de remarquer que l'isomorphisme intermédiaire préserve évidemment le support et que :

- les doubles classes $\Lambda(t, r) w \Lambda(t, r)$, pour $w \in \tilde{W}\left(\mathfrak{B}_{t}\right)$, sont deux à deux disjointes (se placer de nouveau dans $G L(2 r N, F)$ ) ;

- si $\mathcal{A}(t, r)$ a pour support $\Gamma(t, r) \tilde{W}\left(\mathfrak{B}_{t}\right) \Gamma(t, r)$, le sous-espace de $\mathcal{A}(t, r)$ de support contenu dans $\Lambda(t, r) w \Lambda(t, r)\left(w \in \tilde{W}\left(\mathfrak{B}_{t}\right)\right)$ est de dimension 1 ;

- les éléments de $\tilde{W}\left(\mathfrak{B}_{t}\right)$ entrelacent chaque représentation $\gamma(t, r)$.

\section{Des paires Couvrantes Dans $S p(2 t N+2 k, F)$}

Les notations de la partie précédente restent en vigueur, nous supposons de plus la caractéristique résiduelle $p$ de $F$ impaire.

Nous allons montrer dans ce paragraphe comment propager des paires couvrantes de $S p(2 N+2 k, F)$ à $S p(2 t N+2 k, F)$. Nous précisons le contexte en 2.1, énonçons le théorème principal en 2.2, puis définissons en 2.3 le groupe de Coxeter $W_{t}$ qui jouera un rôle essentiel. Le théorème est démontré en 2.4 (les paires proposées sont décomposées), 2.5 (étude des doubles classes des éléments de $W_{t}$ ), 2.6 (certains éléments des algèbres de Hecke impliquées sont inversibles), 2.7 (ces algèbres de Hecke sont isomorphes) et 2.8 (conclusion de la démonstration).

L'entier $N$ et un type simple maximal $(\Gamma, \gamma)$ attaché à une représentation autoduale de $G L(N, F)$ sont fixés une fois pour toutes (les propriétés spécifiques de $(\Gamma, \gamma)$ sont rappelées plus loin en 2.1.c). On aura souvent besoin de comparer les situations dans $S p(2 t N+2 k, F)$ et $S p(2 t N, F)$, de sorte que l'entier $k$ pourra être remplacé par 0. Quant aux entiers $t$ et $r$ (corollaire 1 de la proposition 1), ils pourront varier. En conséquence, les notations seront parfois indexées par $t, k$ ou $r$ en fonction du contexte; ces indices seront omis chaque fois qu'aucune ambiguité ne sera à craindre.

\subsection{Contexte.}

2.1.a. On travaille dans le groupe $G=G_{t, k}=S p(2 t N+2 k, F)$, sur lequel on fixe une mesure de Haar. On utilisera pour les éléments de $M_{t N+2 k+t N}(F)$ diverses notations par blocs que l'on va spécifier dès maintenant. L'espace vectoriel sousjacent $V(t, k)=F^{t N+2 k+t N}$ se décompose en $V_{-t} \oplus V_{0} \oplus V_{t}$, où $V_{-t}$ (resp. $V_{t}$ ) est le sous-espace des vecteurs colonnes dont les $2 k+t N$ dernières (resp. premières) coordonnées sont nulles, et $V_{0} \simeq F^{2 k}$ est le sous-espace des vecteurs colonnes dont les $t N$ premières et $t N$ dernières coordonnées sont nulles. Les conventions de 1.2 s'appliquent à $V_{-t}$ et $V_{t}$, canoniquement isomorphes à $F^{t N}: V_{-t}=\bigoplus_{j=t}^{1} V_{-t}^{(j)}$ et $V_{t}=\bigoplus_{j=t}^{1} V_{t}^{(j)}$. Les décompositions suivantes de l'espace $V(t, k)$ seront utilisées plusieurs fois :

$$
\begin{aligned}
& V(t, k)=F^{t N+2 k+t N}=V_{-t} \oplus V_{0} \oplus V_{t}, \\
& V(t, k)=F^{t N+2 k+t N}=V_{-t}^{(t)} \oplus V(t-1, k) \oplus V_{t}^{(1)}, \\
& V(t, k)=F^{t N+2 k+t N}=\bigoplus_{j=t}^{1} V_{-t}^{(j)} \oplus V_{0} \oplus \bigoplus_{j=t}^{1} V_{t}^{(j)} .
\end{aligned}
$$


Chacune permet d'écrire les éléments de $M_{t N+2 k+t N}(F)$ comme des matrices par blocs et le drapeau sous-jacent à chaque décomposition permet de définir un sousgroupe parabolique. Dans le cas particulier $k=0$ on aura $V_{0}=\{0\}$.

Notons $g \mapsto^{\tau} g, g \in M_{i, j}(F)$, la transposition par rapport à l'antidiagonale :

$$
{ }^{t \tau} g={ }^{\tau t} g=w_{i} g w_{j}, \text { avec } w_{i}=\left(\begin{array}{cccc}
0 & \ldots & 0 & 1 \\
0 & \ldots & 1 & 0 \\
1 & 0 & \ldots & 0
\end{array}\right) \in G L(i, F) .
$$

Le groupe $G$ est vu comme groupe symplectique de $F^{t N+2 k+t N}$ muni d'une forme de matrice $\left(\begin{array}{cccc}0 & 0 & -w_{t N} \\ 0 & w_{2 k} \alpha^{-1} & 0 \\ w_{t N} & 0 & 0\end{array}\right)$ dans la base canonique, où $\alpha \in G L(2 k, F)$ vérifie $\alpha^{\tau} \alpha^{-1}=-I_{2 k}$ (travailler dans le cas standard $\alpha=\left(\begin{array}{cc}I_{k} & 0 \\ 0 & -I_{k}\end{array}\right)$ ne simplifie rien). On note $X \mapsto{ }^{T} X$ l'involution associée sur $M_{t N+2 k+t N}(F)$ :

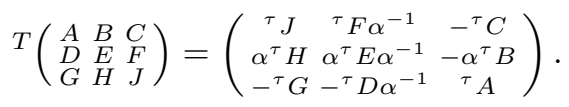

Pour ne pas alourdir les notations, on identifiera au besoin :

- $G_{t, 0}$ à son image dans $G_{t, k}$ via l'inclusion (1) de $V_{-t} \oplus V_{t}$ dans $V(t, k)$;

- $G_{t-1, k}$ à son image dans $G_{t, k}$ via l'inclusion (2) de $V(t-1, k)$ dans $V(t, k)$.

Enfin, les plongements suivants nous seront utiles :

$-\iota: G L(t N, F) \hookrightarrow G_{t, k}$ défini par $\iota(g)=\left(\begin{array}{ccc}g & 0 & 0 \\ 0 & I_{2 k} & 0 \\ 0 & 0 & \tau \\ g^{-1}\end{array}\right)(g \in G L(t N, F)) ;$

$-j_{i}: G L(2 N, F) \hookrightarrow G_{t, k}$ pour $1 \leqslant i \leqslant t-1(t \geqslant 2)$, composé de $\iota$ et de l'injection suivante de $G L(2 N, F)$ dans $G L(t N, F)$ :

$$
\begin{aligned}
& g \mapsto\left(\begin{array}{ccc}
I_{(t-i-1) N} & & \\
& g & \\
& & I_{(i-1) N}
\end{array}\right)(g \in G L(2 N, F)) ; \\
& -j_{0}: G_{1, k} \hookrightarrow G_{t, k} \text { défini par } g \mapsto\left(\begin{array}{ccc}
I_{(t-1) N} & & \\
& g & \\
& & I_{(t-1) N}
\end{array}\right)\left(g \in G_{1, k}\right) \text {; } \\
& -j_{t}: G_{1, k} \hookrightarrow G_{t, k} \text { défini par } j_{t}\left(\left(\begin{array}{lll}
a & b & c \\
d & e & f \\
g & h & i
\end{array}\right)\right)=\left(\begin{array}{ccccc}
a & 0 & b & 0 & c \\
0 & I_{(t-1) N} & 0 & 0 & 0 \\
d & 0 & e & 0 & f \\
0 & 0 & 0 & I_{(t-1) N} & 0 \\
g & 0 & h & 0 & i
\end{array}\right) \text {. }
\end{aligned}
$$

2.1.b. On utilisera les sous-groupes paraboliques suivants de $G_{t, k}$ :

$P=P_{t, k}=M_{t, k} N_{t, k}$ : le sous-groupe parabolique maximal de $G$ formé des matrices par blocs triangulaires supérieures dans la décomposition (1). $N=N_{t, k}$ est son radical unipotent et $M=M_{t, k}$ son facteur de Levi formé des matrices diagonales par blocs, isomorphe à $G L(t N, F) \times S p(2 k, F)$ :

$$
P_{t, k}=M_{t, k} N_{t, k}=\left(\begin{array}{ccc}
G L(t N, F) & * & * \\
0 & S p(2 k, F) & * \\
0 & 0 & G L(t N, F)
\end{array}\right) \cap G_{t, k} .
$$

En particulier, $P_{t, 0}$ est le sous-groupe parabolique de Siegel de $G_{t, 0}$ et le Levi de Siegel $M_{t, 0}$ s'identifie à $G L(t N, F)$.

$Q=Q_{t, k}=L_{t, k} R_{t, k}$ : le sous-groupe parabolique maximal de $G$ formé des matrices par blocs triangulaires supérieures dans la décomposition (2). $R=R_{t, k}$ est son radical unipotent et $L=L_{t, k}$ son facteur de Levi formé des matrices diagonales par blocs, isomorphe à $G L(N, F) \times G_{t-1, k}$ :

$$
Q_{t, k}=L_{t, k} \quad R_{t, k}=\left(\begin{array}{ccc}
G L(N, F) & * & * \\
0 & G_{t-1, k} & * \\
0 & 0 & G L(N, F)
\end{array}\right) \cap G_{t, k} .
$$


$P^{0}=P_{t, k}^{0}=M_{t, k}^{0} N_{t, k}^{0}$ : le sous-groupe parabolique de $G$ formé des matrices par blocs triangulaires supérieures dans la décomposition (3). $N^{0}=N_{t, k}^{0}$ est son radical unipotent et $M^{0}=M_{t, k}^{0}$ son facteur de Levi formé des matrices diagonales par blocs, isomorphe à $G L(N, F) \times \cdots \times G L(N, F) \times S p(2 k, F)$ :

$$
P_{t, k}^{0}=M_{t, k}^{0} N_{t, k}^{0}=\left(\begin{array}{ccccc}
G L(N, F) & * & \ldots & \ldots & * \\
0 & \ddots & & & \vdots \\
\vdots & & S p(2 k, F) & & \vdots \\
\vdots & & & \ddots & \stackrel{*}{*} \\
0 & \ldots & \ldots & 0 & G L(N, F)
\end{array}\right) \cap G_{t, k} .
$$

On notera respectivement $N^{-}=N_{t, k}^{-}, R^{-}=R_{t, k}^{-}$et $N^{0-}=N_{t, k}^{0-}$ les opposés de $N, R$ et $N^{0}$ par rapport à $M, L$ et $M^{0}$.

2.1.c. Soit $(\Gamma, \gamma)$ un type simple maximal dans $G L(N, F)$, dans les notations de 1.2. On suppose $(\Gamma, \gamma)$ attaché à une représentation autoduale de $G L(N, F)$ et on suppose que l'ordre $\mathfrak{A}_{0}$ est $\tau$-stable - remarquons que dans les énoncés de [B13, l'hypothèse $\mathfrak{A}_{0}$ standard peut être remplacée par $\mathfrak{A}_{0} \tau$-stable. Quitte à changer l'élément $\beta$ dans la strate initiale, ce type possède alors les propriétés suivantes (voir Bl3] Proposition 2.2, Corollaire 2.3 et la remarque 2 qui suit ; en niveau 0 , on a $E=F$ et (i) n'est pas valide, mais $\sigma=I$ vérifie (ii), (iii) et (iv)) :

(i) $E=F[\beta]$ est une extension quadratique de $F\left[\beta^{2}\right]$. On note $x \mapsto \bar{x}$ l'élément non trivial du groupe de Galois de $F[\beta]$ sur $F\left[\beta^{2}\right]$.

(ii) Il existe un élément $\sigma$ de $U\left(\mathfrak{A}_{0}\right)$ tel que $\sigma^{-1} x \sigma={ }^{\tau} \bar{x}$ pour tout $x \in E$.

(iii) Les réseaux $\mathfrak{H}^{1}=\mathfrak{H}^{1}\left(\beta, \mathfrak{A}_{0}\right)$ et $\mathfrak{J}^{0}=\mathfrak{J}^{0}\left(\beta, \mathfrak{A}_{0}\right)$ sont stables par l'application $x \mapsto \sigma^{\tau} x \sigma^{-1}$. Les paires $\left(H^{1}=H^{1}\left(\beta, \mathfrak{A}_{0}\right), \theta_{0}\right),\left(J^{1}=J^{1}\left(\beta, \mathfrak{A}_{0}\right), \eta_{0}\right)$ et $(\Gamma, \gamma)$ sont stables par $x \mapsto \sigma^{\tau} x^{-1} \sigma^{-1}$ (le groupe est stable et la représentation transformée en une représentation équivalente).

(iv) Les éléments $\sigma^{\tau} \sigma^{-1}$ et $\varpi_{E}^{-1} \sigma^{\tau} \varpi_{E}^{\tau} \sigma^{-1}$ appartiennent à $\Gamma$.

Si $\xi$ est une représentation d'un sous-groupe $H$ de $G L(i, F)$, on notera $\xi^{*}$ la représentation de ${ }^{\tau} H$ définie par $\xi^{*}(g)=\xi\left({ }^{\tau} g^{-1}\right), g \in{ }^{\tau} H$. La propriété (iii) exprime en particulier l'équivalence des représentations $\gamma^{*}$ et $\gamma^{\sigma}$.

2.1.d. Reprenons la paire couvrante $(\Gamma(2 t, r), \gamma(2 t, r))$ de 1.3 Corollaire 1 en dimension $2 t N$ et écrivons le groupe sous forme de matrices $2 \times 2$ par blocs de taille $t N$ :

$$
\Gamma(2 t, r)=\left(\begin{array}{cc}
\Gamma(t, t) & \Gamma^{+}(t, r) \\
\Gamma^{-}(t, r) & \Gamma(t, t)
\end{array}\right) \quad t \leqslant r \leqslant 2 t
$$

Ceci définit $\Gamma^{+}(t, r)$ et $\Gamma^{-}(t, r)$; on a par exemple :

$$
\Gamma^{+}(1,1)=\varpi_{E}^{-1} \mathfrak{H}^{1}, \Gamma^{+}(1,2)=\mathfrak{J}^{0}, \Gamma^{-}(1,2)=\mathfrak{H}^{1} \text { et } \Gamma^{-}(1,1)=\varpi_{E} \mathfrak{J}^{0} .
$$

Par transitivité des paires couvrantes (1.1), la paire $(\Gamma(2 t, r), \gamma(2 t, r))$ est une paire couvrante de $(\Gamma(t, t) \times \Gamma(t, t), \gamma(t, t) \otimes \gamma(t, t))$ dans $G L(2 t N, F)$ relativement à $P_{t, 0}$. Conjuguons cette paire comme dans [B]3] 2.2 par

$$
\Sigma=\Sigma_{t}=\operatorname{diag}\left(I_{N}, \cdots, I_{N}, \sigma, \cdots, \sigma\right)
$$

(les $t$ premiers blocs diagonaux sont égaux à $I_{N}$ et les $t$ suivants à $\sigma$ ). Grâce aux propriétés rappelées en 2.1.c, il est facile de vérifier qu'on obtient une paire couvrante $\left(\Gamma(2 t, r)^{\Sigma}, \gamma(2 t, r)^{\Sigma}\right)$ de $\left(\Gamma(t, t) \times{ }^{\tau} \Gamma(t, t), \gamma(t, t) \otimes \gamma(t, t)^{*}\right)$. On peut dès lors appliquer la proposition 1.2 de $\mathrm{Bl} 3$, qui affirme que : 
L'intersection $\Omega(t, r, 0)$ de $\Gamma(2 t, r)^{\Sigma}$ avec $S p(2 t N, F)$ porte une paire décomposée au-dessus de $(\iota(\Gamma(t, t)), \gamma(t, t))$ relativement à $P_{t, 0}$.

Il est agréable de visualiser les choses encore une fois sous forme de matrices par blocs. On identifie $M_{a N, b N}(F)$ à $M_{a, b}\left(M_{N}(F)\right)$ de sorte que multiplier $X \in$ $M_{a N, b N}(F)$ par $\sigma$ à droite (ou à gauche) signifie multiplier chacune de ses entrées, éléments de $M_{N}(F)$, par $\sigma$ à droite (ou à gauche). Avec ces conventions :

$$
\Omega(t, r, 0)=\left(\begin{array}{cc}
\Gamma(t, t) & \Gamma^{+}(t, r) \sigma \\
\sigma^{-1} \Gamma^{-}(t, r) & \tau \\
& \Gamma(t, t)
\end{array}\right) \cap G_{t, 0} .
$$

Il s'avère que la paire décomposée portée par $\Omega(t, r, 0)$ est couvrante (voir exemple 3.4.b) : les groupes $\Omega(t, r, 0)$ sont des cas particuliers des groupes $\Omega(t, r, k)$ définis au paragraphe suivant.

2.2. Le théorème principal et ses hypothèses. On fixe dans $S p(2 k, F)$ une paire $(\Delta, \delta)$ formée d'un sous-groupe ouvert compact $\Delta$ de $S p(2 k, F)$ et d'une représentation lisse irréductible $\delta$ de $\Delta$. Par ailleurs $(\Gamma, \gamma)$ est un type simple maximal dans $G L_{N}(F)$, attaché à une représentation autoduale, et choisi comme en 2.1.c.

On définit les éléments suivants de $G_{t, k}$ :

$$
s(t, k)=\left(\begin{array}{ccc}
0 & 0 & \sigma \\
0 & I_{2 k+2(t-1) N} & 0 \\
-\tau \sigma^{-1} & 0 & 0
\end{array}\right), \quad q(t, k)=\left(\begin{array}{ccc}
0 & 0 & -{ }^{\tau} \sigma^{\tau} \varpi_{E}^{-1} \\
0 & I_{2 k+2(t-1) N} & 0 \\
\sigma^{-1} \varpi_{E} & 0 & 0
\end{array}\right) .
$$

Voici les conditions dans lesquelles nous pouvons propager des paires couvrantes :

(H1) Il existe des $\mathfrak{o}_{F}$-réseaux $\Delta^{+}$de $M_{N, 2 k}(F)$ et $\Delta^{-}$de $M_{2 k, N}(F)$ tels que les sous-ensembles $\Omega(1,1, k)$ et $\Omega(1,2, k)$ de $G_{1, k}$ définis par :

$$
\Omega(1,1, k)=\left(\begin{array}{ccc}
\Gamma & \Delta^{+} & \varpi_{E}^{-1} \mathfrak{H}^{1} \sigma \\
\Delta^{-} & \Delta & \alpha^{\tau} \Delta^{+} \\
\sigma^{-1} \mathfrak{J}^{0} \varpi_{E} & { }^{\tau} \Delta^{-} \alpha^{-1} & { }^{\tau} \Gamma
\end{array}\right) \cap G_{1, k}
$$

et

$$
\Omega(1,2, k)=\left(\begin{array}{ccc}
\Gamma & \Delta^{+} & \mathfrak{J}^{0} \sigma \\
\Delta^{-} & \Delta & \alpha^{\tau} \Delta^{+} \\
\sigma^{-1} \mathfrak{H}^{1} & { }^{\tau} \Delta^{-} \alpha^{-1} & { }^{\tau} \Gamma
\end{array}\right) \cap G_{1, k}
$$

soient des sous-groupes de $G_{1, k}$ qui portent des paires décomposées, notées respectivement $(\Omega(1,1, k), \omega(1,1, k))$ et $(\Omega(1,2, k), \omega(1,2, k))$, au-dessus de $(\iota(\Gamma) \times \Delta, \gamma \otimes \delta)$ relativement à $P_{1, k}$.

(H2) L'algèbre de Hecke $\mathcal{H}\left(G_{1, k}, \omega(1,2, k)\right)$ contient un élément $e_{s(1, k)}$ tel que :

$$
\left\{\begin{array}{l}
\operatorname{Supp} e_{s(1, k)}=\Omega(1,2, k) s(1, k) \Omega(1,2, k), \\
e_{s(1, k)}^{2}=a_{s} e_{s(1, k)}+b_{s} \mathcal{I} \quad \text { avec } a_{s} \in \mathbb{C}, b_{s} \in \mathbb{C}^{\times} .
\end{array}\right.
$$

(H3) L'algèbre de Hecke $\mathcal{H}\left(G_{1, k}, \omega(1,1, k)\right)$ contient un élément $e_{q(1, k)}$ tel que :

$$
\left\{\begin{array}{l}
\text { Supp } e_{q(1, k)}=\Omega(1,1, k) q(1, k) \Omega(1,1, k), \\
e_{q(1, k)}^{2}=a_{q} e_{q(1, k)}+b_{q} \mathcal{I} \quad \text { avec } a_{q} \in \mathbb{C}, b_{q} \in \mathbb{C}^{\times} .
\end{array}\right.
$$


Théorème 1. Sous les hypothèses (H1), (H2) et (H3), on définit, pour tout $t \geqslant 1$ et tout $r$ tel que $t \leqslant r \leqslant 2 t$, un sous-ensemble $\Omega(t, r, k)$ de $G_{t, k}$ par :

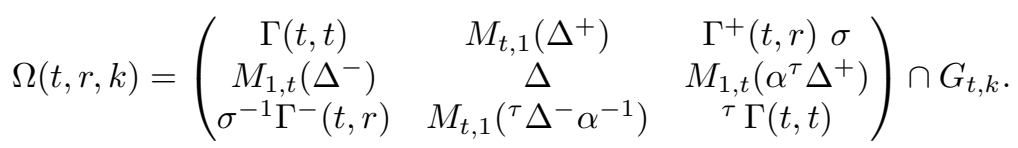

Alors $\Omega(t, r, k)$ est un sous-groupe de $G_{t, k}$ qui porte une paire couvrante $(\Omega(t, r, k), \omega(t, r, k))$ de $\left(\iota\left(\Gamma^{\times t}\right) \times \Delta, \gamma^{\otimes t} \otimes \delta\right)$ relativement à $P_{t, k}^{0}$.

Par transitivité des paires couvrantes, la paire $(\Omega(t, r, k), \omega(t, r, k))$ est aussi une paire couvrante de $(\iota(\Gamma(t, t)) \times \Delta, \gamma(t, t) \otimes \delta)$ relativement à $P_{t, k}(1.1)$.

La démonstration de ce théorème occupe toute la suite de la partie 2, le paragraphe 2.8 en résume les éléments essentiels. Ceci sera complété dans la partie 3 par une description de l'algèbre de Hecke attachée à $(\Omega(t, r, k), \omega(t, r, k))$.

Notation. On convient d'étendre les notations $\Omega(t, r, k), \Gamma^{+}(t, r)$ etc., aux entiers $r$ supérieurs à $2 t$ en posant $\Omega(t, r, k)=\Omega(t, 2 t, k)$ pour $r \geqslant 2 t$, etc.

2.3. Le groupe de Coxeter sous-jacent. Soit $\left(\mathbf{W}_{t}, \mathbf{S}_{t}\right)$ le groupe de Coxeter de type $\tilde{C}_{t}$, défini par générateurs et relations comme suit : les générateurs sont les éléments de $\mathbf{S}_{t}=\left\{\mathbf{s}_{0}, \mathbf{s}_{1}, \ldots \mathbf{s}_{t}\right\}$ et les relations sont $\mathbf{s}_{i}^{2}=1$ pour $0 \leqslant i \leqslant t$, et les relations de tresse:

$$
\left\{\begin{array}{l}
\mathbf{s}_{i} \mathbf{s}_{j}=\mathbf{s}_{j} \mathbf{s}_{i} \quad \text { si } \mid i-j \geqslant 2, \\
\mathbf{s}_{i} \mathbf{s}_{i+1} \mathbf{s}_{i}=\mathbf{s}_{i+1} \mathbf{s}_{i} \mathbf{s}_{i+1} \quad \text { si } 1 \leqslant i \leqslant t-1, \\
\mathbf{s}_{0} \mathbf{s}_{1} \mathbf{s}_{0} \mathbf{s}_{1}=\mathbf{s}_{1} \mathbf{s}_{0} \mathbf{s}_{1} \mathbf{s}_{0} \quad \text { si } t>1, \\
\mathbf{s}_{t} \mathbf{s}_{t-1} \mathbf{s}_{t} \mathbf{s}_{t-1}=\mathbf{s}_{t-1} \mathbf{s}_{t} \mathbf{s}_{t-1} \mathbf{s}_{t} \quad \text { si } t>1 .
\end{array}\right.
$$

On notera $l$ la longueur dans le système de Coxeter $\left(\mathbf{W}_{t}, \mathbf{S}_{t}\right)$ et $\left(\mathbf{W}_{t}^{0}, \mathbf{S}_{t}^{0}\right)$ le sousgroupe engendré par $\mathbf{S}_{t}^{0}=\left\{\mathbf{s}_{0}, \mathbf{s}_{1}, \ldots \mathbf{s}_{t-1}\right\}$.

Pour $1 \leqslant i \leqslant t-1$ on pose $s_{i}=j_{i}\left(\left(\begin{array}{cc}0 & I_{N} \\ I_{N} & 0\end{array}\right)\right) \in G_{t, k}$. Soit $s_{t}=q(t, k)$ et soit $s_{0}=j_{0}(s(1, k))$. On notera $S_{t}=\left\{s_{0}, s_{1}, \ldots s_{t}\right\}$ et $S_{t}^{0}=\left\{s_{0}, s_{1}, \ldots s_{t-1}\right\}$.

On vérifie aisément que les éléments $s_{0}, s_{1}, \ldots s_{t}$ vérifient les relations de tresse ci-dessus, de sorte que l'application $\mathbf{s}_{i} \mapsto s_{i}(0 \leqslant i \leqslant t)$ se prolonge de manière unique en une application de $\mathbf{W}_{t}$ dans $G_{t, k}$, qui à $\mathbf{w} \in \mathbf{W}_{t}$ de décomposition réduite $\mathbf{w}=\mathbf{s}_{i_{1}} \mathbf{s}_{i_{2}} \cdots \mathbf{s}_{i_{z}}$ associe $w=s_{i_{1}} s_{i_{2}} \cdots s_{i_{z}}$ ([B], IV.1.5). L'image $W_{t}$ de cette application est contenue dans le normalisateur du sous-groupe de Levi $M_{t, k}^{0}$ et l'image $W_{t}^{0}$ de $\mathbf{W}_{t}^{0}$ fournit ([G] $)$ un système de représentants du quotient

$$
N_{G_{t, k}}\left(M_{t, k}^{0}\right) / M_{t, k}^{0} \simeq \mathbf{W}_{t}^{0} .
$$

Notons $\Omega_{M^{0}}(t, k)$ l'intersection de (l'ensemble) $\Omega(t, r, k)$ avec $M_{t, k}^{0}$, isomorphe à $\Gamma^{\times t} \times \Delta$, et $\omega_{M^{0}}(t, k)$ la représentation $\gamma^{\otimes t} \otimes \delta$ de ce sous-groupe. On s'intéresse à leur normalisateur dans $G_{t, k}$.

Proposition 3. Le groupe $N_{G_{t, 0}}\left(\Omega_{M^{0}}(t, 0), \omega_{M^{0}}(t, 0)\right) / \Omega_{M^{0}}(t, 0)=\bar{W}_{t}$ est un groupe de Coxeter de type $\tilde{C}_{t}$ dont $W_{t}$ est un système de représentants.

Le groupe $N_{G_{t, k}}\left(\Omega_{M^{0}}(t, k), \omega_{M^{0}}(t, k)\right) / \Omega_{M^{0}}(t, k)$ est produit direct de $\bar{W}_{t}$ par $N_{S p(2 k, F)}(\Delta, \delta) / \Delta$.

Démonstration. La notation $N_{S p(2 k, F)}(\Delta, \delta)$ (par exemple) désigne le sous-groupe de $N_{S p(2 k, F)}(\Delta)$ formé des éléments $x$ tels que les représentations $\delta$ et $\delta^{x}$ soient 
équivalentes. Comme les éléments $s_{i}$ normalisent $\Omega_{M^{0}}(t, k)$ et vérifient $\left.\omega_{M^{0}}(t, k)\right) \simeq$ $\left.\omega_{M^{0}}(t, k)\right)^{s_{i}}$ (voir 2.1.c et [B13]), l'ensemble $W_{t}$ est contenu dans

$$
\mathcal{N}=N_{G_{t, k}}\left(\Omega_{M^{0}}(t, k), \omega_{M^{0}}(t, k)\right) .
$$

Un élément $g$ de $\mathcal{N}$ doit normaliser $M_{t, k}^{0}$, il y a donc un élément $w$ de $W_{t}^{0}$ tel que $w^{-1} g$ appartienne à $\mathcal{N} \cap M_{t, k}^{0}$. Les blocs diagonaux de $w^{-1} g$ de type $G L(N, F)$ appartiennent donc à $\varpi_{E}^{\mathbb{Z}} \Gamma$ ou à son anti-transposé ([BK1] 6.1.2) et le bloc diagonal de type $S p(2 k, F)$ appartient à $N_{S p(2 k, F)}(\Delta, \delta)$. Comme les éléments de la forme $\iota\left(\operatorname{diag}\left(\varpi_{E}^{j_{t}}, \ldots, \varpi_{E}^{j_{1}}\right)\right)$ appartiennent à $W_{t} \Omega_{M^{0}}(t, k)$ (vérification immédiate avec 2.1.c (iv)) on a bien, si $k=0, \mathcal{N}=W_{t} \Omega_{M^{0}}(t, 0)$.

Enfin, l'injectivité de la composition $\mathbf{W}_{t} \rightarrow W_{t} \rightarrow \mathcal{N} / \Omega_{M^{0}}(t, k)$ est claire (tout élément de $W_{t}$ s'écrit de manière unique comme produit d'un élément de $W_{t}^{0}$ par un élément de $\left.W_{t} \cap M_{t, k}^{0}\right)$.

Remarque. Soit $B_{2 t}^{\Sigma}$ le commutant de $E^{\Sigma}$ dans $M_{2 t N}(F)$ (voir 1.2 et 2.1.d). On a $\Omega(t, r, 0) \cap B_{2 t}^{\Sigma}=\mathcal{J}^{\Sigma}$ où $\mathcal{J}$ est le sous-groupe parahorique suivant de $B_{2 t}$ :

$$
\mathcal{J}=\left(\begin{array}{cccc}
\mathfrak{B}_{0}^{\times} & \mathfrak{B}_{0} & \ldots & \mathfrak{B}_{0} \\
\mathfrak{Q}_{0} & \mathfrak{B}_{0}^{\times} & \ddots & \vdots \\
\vdots & \ddots & \ddots & \vdots \\
\mathfrak{Q}_{0} & \ldots & \mathfrak{Q}_{0} & \mathfrak{B}_{0}^{\times}
\end{array}\right) .
$$

Soit $G_{t, 0}^{E}=G_{t, 0} \cap B_{2 t}^{\Sigma}$ et soit $\Omega_{M^{0}}^{E}=\Omega_{M^{0}}(t, 0) \cap B_{2 t}^{\Sigma}$. Alors $W_{t}$ est aussi un système de représentants dans $G_{t, 0}^{E}$ du quotient $N_{G_{t, 0}^{E}}\left(\Omega_{M^{0}}^{E}\right) / \Omega_{M^{0}}^{E}$.

\subsection{Propagation de paires décomposées.}

Proposition 4. Sous l'hypothèse (H1), l'ensemble $\Omega(t, r, k)$ est un groupe qui porte une paire décomposée $(\Omega(t, r, k), \omega(t, r, k))$ au-dessus de $\left(\iota\left(\Gamma^{\times t}\right) \times \Delta, \gamma^{\otimes t} \otimes \delta\right)$ relativement $\grave{a} P_{t, k}^{0}$.

Démonstration. Montrer qu'une paire est décomposée n'est pas très amusant, mais c'est fondamental. On va montrer que $\Omega(t, r, k)$ porte une paire décomposée audessus de $(\iota(\Gamma(t, t)) \times \Delta, \gamma(t, t) \otimes \delta)$ relativement à $P_{t, k}$; cela entraîne l'assertion de la proposition par transitivité (voir 1.1), puisque $(\Gamma(t, t) \times \Delta, \gamma(t, t) \otimes \delta$ ) est elle-même décomposée au-dessus de $\left(\Gamma^{\times t} \times \Delta, \gamma^{\otimes t} \otimes \delta\right)$ (Corollaire 1). Noter que le cas $k=0$ a été traité en 2.1.d. Pour le cas général on procède par récurrence sur $t$ : l'assertion est vraie pour $t=1$, c'est l'hypothèse (H1) ; on la suppose vraie jusqu'au rang $t-1$ et on l'établit au rang $t$.

L'ensemble $\Omega=\Omega(t, r, k)$ est tel que $\Omega \cap M=\Gamma(t, t) \times \Delta$. C'est un groupe qui porte une paire décomposée au-dessus de $(\iota(\Gamma(t, t)) \times \Delta, \gamma(t, t) \otimes \delta)$ relativement à $P=P_{t, k}$ si et seulement si ([B]1]) :

(i) Les intersections $\Omega \cap N^{-}$et $\Omega \cap N$ sont des groupes normalisés par $\Omega \cap M$.

(ii) Le produit $\left(\Omega \cap N^{-}\right)(\operatorname{Ker} \gamma(t, t) \times \operatorname{Ker} \delta)(\Omega \cap N)$ est stable par produit à gauche par $(\Omega \cap N)$.

Commençons par vérifier (i). Le fait que $\Omega \cap N^{-}$et $\Omega \cap N$ soient des groupes se ramène aux conditions

$$
\Delta^{+} \alpha^{\tau} \Delta^{+} \subset \mathfrak{J}^{0} \sigma \quad \text { et } \quad{ }^{\tau} \Delta^{-} \alpha^{-1} \Delta^{-} \subset \sigma^{-1} \mathfrak{J}^{0} \varpi_{E}
$$


qui sont forcément vérifiées vu l'hypothèse (H1). La normalisation par $\Omega \cap M$ est acquise pourvu que

$$
\mathfrak{J}^{0} \Delta^{+} \subset \Delta^{+} \quad \text { et } \quad \Delta^{-} \mathfrak{J}^{0} \subset \Delta^{-} .
$$

Or (H1) assure que $\Gamma \Delta^{+} \subset \Delta^{+}$et $\Delta^{-} \Gamma \subset \Delta^{-}$. On passe de $\Gamma$ à $\mathfrak{J}^{0}$ comme dans B13. Lemme 2.2, en utilisant le fait que $\mathfrak{B}_{0}=\mathfrak{o}_{E}\left[\mathfrak{B}_{0}^{\times}\right]$([B] p. 190).

Passons à (ii). Le point essentiel est l'usage de plusieurs paraboliques en même temps ; de fait, on a (voir la fin de 1.1) :

$$
\begin{aligned}
& \Omega \cap N=(\Omega \cap N \cap L) \rtimes(\Omega \cap N \cap R), \\
& \Omega \cap N^{-}=\left(\Omega \cap N^{-} \cap L\right) \rtimes\left(\Omega \cap N^{-} \cap R^{-}\right), \\
& \Omega \cap R=(\Omega \cap R \cap M) \rtimes(\Omega \cap R \cap N), \\
& \Omega \cap R^{-}=\left(\Omega \cap R^{-} \cap M\right) \rtimes\left(\Omega \cap R^{-} \cap N^{-}\right) .
\end{aligned}
$$

Il suffit donc dans (ii) de montrer séparément la stabilité par produit à gauche par $\Omega \cap N \cap L$ et $\Omega \cap N \cap R$. La première stabilité est facile :

$$
\begin{aligned}
& (\Omega \cap N \cap L)\left(\Omega \cap N^{-}\right)=(\Omega \cap N \cap L)\left(\Omega \cap N^{-} \cap L\right)\left(\Omega \cap N^{-} \cap R^{-}\right) \\
& \quad \subset\left(\Omega \cap N^{-} \cap L\right)\left(I_{N} \times \operatorname{Ker} \gamma(t-1, t-1) \times \operatorname{Ker} \delta\right)(\Omega \cap N \cap L)\left(\Omega \cap N^{-} \cap R^{-}\right)
\end{aligned}
$$

par l'hypothèse de récurrence appliquée à $\Omega \cap L=\Gamma \times \Omega(t-1, r, k)$ dans $L \simeq$ $G L(N, F) \times G_{t-1, k}$. En outre $L$ normalise $R^{-}$donc $(\Omega \cap N \cap L)\left(\Omega \cap N^{-} \cap R^{-}\right)$est contenu dans $\left(\Omega \cap R^{-}\right)(\Omega \cap N \cap L)=\left(\Omega \cap R^{-} \cap N^{-}\right)\left(\Omega \cap R^{-} \cap M\right)(\Omega \cap N \cap L)$. Le terme médian $\Omega \cap R^{-} \cap M$ est contenu dans Ker $\gamma(t, t) \times \operatorname{Ker} \delta$ vu la définition de $R$-les blocs diagonaux sont unipotents inférieurs par blocs - d'où le résultat.

La seconde stabilité est plus fastidieuse et on ne donnera pas tous les détails. On reproduit tout de même le calcul à la base de toutes les vérifications :

$$
\begin{gathered}
\left(\begin{array}{ccc}
I_{i} & A & B \\
0 & I_{2 j} & A^{*} \\
0 & 0 & I_{i}
\end{array}\right)\left(\begin{array}{ccc}
I_{i} & 0 & 0 \\
-X^{*} & I_{2 j} & 0 \\
Y & X & I_{i}
\end{array}\right)=\left(\begin{array}{ccc}
I_{i}-A X^{*}+B Y & A+B X & B \\
-X^{*}+A^{*} Y & I_{2 j}+A^{*} X & A^{*} \\
Y & X & I_{i}
\end{array}\right), \\
\left(\begin{array}{cccc}
I_{i} & 0 & 0 \\
-X^{\prime *} & I_{2 j} & 0 \\
Y^{\prime} & X^{\prime} & I_{i}
\end{array}\right)\left(\begin{array}{ccc}
g & 0 & 0 \\
0 & h & 0 \\
0 & 0 & 0
\end{array}\right)\left(\begin{array}{ccc}
I_{i} & A^{\prime} & B^{\prime} \\
0 & I_{2 j} & A^{\prime *} \\
0 & 0 & I_{i}
\end{array}\right)=\left(\begin{array}{ccc}
g & g A^{\prime} & g B^{\prime} \\
-X^{\prime *} g & h-X^{\prime *} g A^{\prime} & \ldots \\
Y^{\prime} g & \cdots & \cdots
\end{array}\right) .
\end{gathered}
$$

On décompose encore $\Omega \cap N \cap R$ sous la forme $\Omega \cap N \cap R=\mathcal{N}_{1} \mathcal{N}_{2}$ avec :

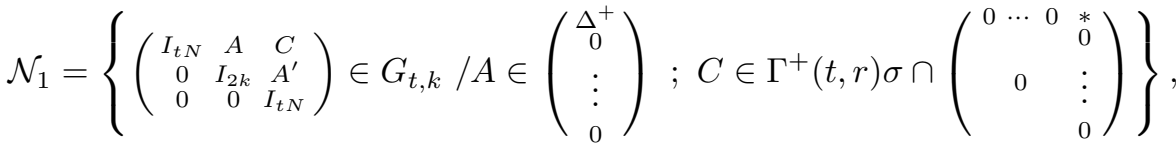

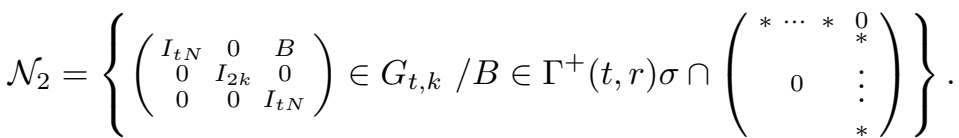

$\mathcal{N}_{1}$ et $\mathcal{N}_{2}$ sont des sous-groupes générateurs de $\Omega \cap N \cap R$ et $\mathcal{N}_{2}$ y est central. On décompose de même : $\Omega \cap N^{-} \cap R^{-}=\mathcal{N}_{1}^{-} \mathcal{N}_{2}^{-}$. On va montrer dans (ii) la stabilité $\operatorname{par} \mathcal{N}_{1}$ et $\mathcal{N}_{2}$ à gauche. Commençons par $\mathcal{N}_{2} ;$ on a :

$$
\mathcal{N}_{2}\left(\Omega \cap N^{-}\right)=\mathcal{N}_{2}\left(\Omega \cap N^{-} \cap R^{-}\right)\left(\Omega \cap N^{-} \cap L\right)=\mathcal{N}_{2} \mathcal{N}_{2}^{-} \mathcal{N}_{1}^{-}\left(\Omega \cap N^{-} \cap L\right) .
$$

Pour le produit $\mathcal{N}_{2} \mathcal{N}_{2}^{-}$, tout se passe dans $G_{t, 0}$ dans lequel on a une paire décomposée (2.1.d), d'où $\mathcal{N}_{2} \mathcal{N}_{2}^{-} \subset\left(\Omega \cap N^{-}\right)(\operatorname{Ker} \gamma(t, t) \times \operatorname{Ker} \delta)\left(\Omega \cap N \cap G_{t, 0}\right)$; on vérifie via $(*)$ que le terme de droite appartient encore à $\mathcal{N}_{2}$, soit

$$
\mathcal{N}_{2}\left(\Omega \cap N^{-}\right) \subset\left(\Omega \cap N^{-}\right)(\operatorname{Ker} \gamma(t, t) \times \operatorname{Ker} \delta) \mathcal{N}_{2} \mathcal{N}_{1}^{-}\left(\Omega \cap N^{-} \cap L\right) .
$$


On examine alors le produit $\mathcal{N}_{2} \mathcal{N}_{1}^{-}$en utilisant $(*)$ et ses notations : il s'agit d'identifier les deux lignes de $(*)$. Or $g=I_{t N}+B Y$ appartient à $\Gamma(t, t)$ par 2.1.d et est de la forme $g=\left(\begin{array}{cc}I_{N} & 0 \\ * & I_{(t-1) N}\end{array}\right)$, de sorte que $X^{\prime}=X$ et $Y^{\prime}=Y$. D'autre part $h=I_{2 k} \operatorname{donc} \operatorname{diag}\left(g, h,{ }^{\tau} g^{-1}\right)$ appartient au noyau de $\gamma(t, t) \otimes \delta$. Vérifions maintenant que $\left(\begin{array}{ccc}I_{t N} & A^{\prime} & B^{\prime} \\ 0 & I_{2 k} & A^{\prime *} \\ 0 & 0 & I_{t N}\end{array}\right)$ appartient à $\Omega \cap N$. On a $A^{\prime}=g^{-1} B X$, ses entrées (blocs $N \times 2 k$ ) appartiennent à $\mathfrak{J}^{0} \sigma^{\tau} \Delta^{-} \alpha^{-1}$ ou $\varpi_{E}^{-1} \mathfrak{H}^{1} \sigma^{\tau} \Delta^{-} \alpha^{-1}$, d'où la condition

$$
\varpi_{E}^{-1} \mathfrak{H}^{1} \sigma^{\tau} \Delta^{-} \alpha^{-1} \subset \Delta^{+}
$$

qui est vérifiée par hypothèse puisque $\Omega(1,1, k)$ est décomposée. La vérification pour $B^{\prime}$ est immédiate (voir 1.3, Lemme 1).

Il reste :

$$
\begin{aligned}
\mathcal{N}_{2}\left(\Omega \cap N^{-}\right) & \subset\left(\Omega \cap N^{-}\right)(\operatorname{Ker} \gamma(t, t) \otimes \delta)(\Omega \cap N)\left(\Omega \cap N^{-} \cap L\right) \\
& \subset\left(\Omega \cap N^{-}\right)(\operatorname{Ker} \gamma(t, t) \otimes \delta)(\Omega \cap N \cap L)(\Omega \cap N \cap R)\left(\Omega \cap N^{-} \cap L\right) \\
& \subset\left(\Omega \cap N^{-}\right)(\operatorname{Ker} \gamma(t, t) \otimes \delta)(\Omega \cap N \cap L)\left(\Omega \cap N^{-} \cap L\right)(\Omega \cap R)
\end{aligned}
$$

(car $L$ normalise $R$ ).

Il n'y a plus qu'à utiliser de nouveau l'hypothèse de récurrence dans $\Omega \cap L$ et la décomposition de $\Omega \cap R$.

La stabilité par $\mathcal{N}_{1}$ se montre de façon analogue et fait intervenir la condition

$$
\alpha^{\tau} \Delta^{+} \sigma^{-1} \mathfrak{H}^{1} \subset \Delta^{-}
$$

vérifiée car $\Omega(1,2, k)$ est décomposée. On omet les détails.

Corollaire 3. La paire $(\Omega(t, r, k), \omega(t, r, k))$ est décomposée au-dessus de $\left(\iota\left(\Gamma^{\times t}\right) \times\right.$ $\left.\Delta, \gamma^{\otimes t} \otimes \delta\right)$ relativement à $w P^{0} w^{-1}$ pour tout élément $w$ de $N_{G}\left(M^{0}\right)$.

Démonstration. La démonstration par récurrence sur la longueur de l'image de $w$ dans $N_{G}\left(M^{0}\right) / M^{0} \simeq \mathbf{W}_{t}^{0}$ est immédiate, une fois établie l'assertion :

Soit $J$ un sous-groupe de $G_{t, k}$ défini par blocs comme en $1.1(\ddagger)$ relativement à la décomposition (3) de $V$ en 2.1.a. Soit $s \in S_{t}^{0}$. Si $J$ porte une paire décomposée au-dessus de $M^{0}$ relativement à $P^{0}$, alors $J$ porte une paire décomposée au-dessus de $M^{0}$ relativement à $s P^{0} s^{-1}$.

En effet, par transitivité (voir 1.1), $J$ porte une paire décomposée au-dessus de $M^{0}(s)$, sous-groupe de Levi de $G$ engendré par $M^{0}$ et $s$, relativement à $P^{0}(s)=$ $M^{0}(s) N^{0}$, de radical unipotent $N^{0}(s)$. Comme $s \in M^{0}(s)$ on en déduit :

$$
s^{-1} J s=s^{-1} J s \cap N^{0}(s)^{-} . s^{-1} J s \cap M^{0}(s) . s^{-1} J s \cap N^{0}(s) .
$$

D'autre part $J \cap M^{0}(s)$ est également décomposée et la conjugaison par $s$ échange $M^{0}(s) \cap N^{0}$ et $M^{0}(s) \cap N^{0^{-}}$, d'où

$$
s^{-1} J s \cap M^{0}(s)=s^{-1} J s \cap M^{0}(s) \cap N^{0^{-}} . s^{-1} J s \cap M^{0} . s^{-1} J s \cap M^{0}(s) \cap N^{0}
$$

et le résultat.

2.5. Quelques propriétés des doubles classes. L'hypothèse (H1) est en vigueur dans tout ce paragraphe, la proposition 4 est donc valide. On travaillera constamment dans la suite avec les éléments de $W_{t}$, l'image de $\mathbf{W}_{t}$ décrite en 2.3 sur laquelle la longueur $l$ de $\mathbf{W}_{t}$ se transporte. On raccourcit les notations en

$$
\mathcal{H}(t, r, k)=\mathcal{H}\left(G_{t, k}, \omega(t, r, k)\right) .
$$


Lemme 2. Soit $w \in W_{t}$. La double classe $\Omega(t, r, k) w \Omega(t, r, k)$ supporte un élément non nul et un seul de $\mathcal{H}(t, r, k)$ à scalaire non nul près.

Notation. On note un tel élément $E_{w}^{(r)}$ ou $E_{w}$, la normalisation ne sera précisée que quand elle sera nécessaire.

Démonstration. Les paires $(\Omega, \omega)$ et $\left(\Omega^{w}, \omega^{w}\right)$ sont toutes deux décomposées relativement à $w^{-1} P^{0} w$ (Corollaire 3 ci-dessus). L'intersection $\Omega \cap \Omega^{w}$ s'obtient donc par intersection terme à terme :

$\Omega \cap \Omega^{w}=\left[\Omega \cap N^{0^{-w}} \cap\left(\Omega \cap N^{0^{-}}\right)^{w}\right]\left[\Omega \cap M^{0} \cap\left(\Omega \cap M^{0}\right)^{w}\right]\left[\Omega \cap N^{0^{w}} \cap\left(\Omega \cap N^{0}\right)^{w}\right]$.

Comme $\omega$ et $\omega^{w}$ sont triviales sur les intersections avec $N^{0^{-w}}$ et $N^{0^{w}}$, il reste :

$$
\operatorname{Hom}_{\Omega \cap \Omega^{w}}\left(\omega, \omega^{w}\right) \simeq \operatorname{Hom}_{\iota\left(\Gamma^{\times t}\right) \times \Delta}\left(\left(\gamma^{\otimes t} \otimes \delta\right),\left(\gamma^{\otimes t} \otimes \delta\right)^{w}\right) \simeq \mathbb{C}
$$

puisque les représentations sont irréductibles et équivalentes (Proposition 3).

L'intérêt essentiel de travailler avec la famille $(\Omega(t, r, k), \omega(t, r, k))_{t \leqslant r \leqslant 2 t}$ de paires décomposées est que les calculs de doubles classes se simplifient à condition de bien choisir $r$. Commençons par des propriétés des générateurs.

Lemme 3. On a les égalités suivantes:

$$
\begin{aligned}
\Omega(t, 2 t, k) s_{i} \Omega(t, 2 t, k) & =\Omega(t, 2 t, k) s_{i} j_{i}\left(\left(\begin{array}{cc}
I_{N} & \mathfrak{J}^{0} \\
0 & I_{N}
\end{array}\right)\right) \\
\Omega(t, 2 t, k) s_{0} \Omega(t, 2 t, k) & =\Omega(t, 2 t, k) s_{0} j_{0}\left(\left(\begin{array}{ccc}
I_{N} & \Delta^{+} & \mathfrak{J}^{0} \sigma \\
0 & I_{2 k} & \alpha^{\tau} \Delta^{+} \\
0 & 0 & I_{N}
\end{array}\right)\right), \\
\Omega(t, t, k) s_{t} \Omega(t, t, k) & =\Omega(t, t, k) s_{t} j_{t}\left(\left(\begin{array}{ccc}
I_{N} & 0 & 0 \\
\Delta^{-} & I_{2 k} & 0 \\
\sigma^{-1} \mathfrak{J}^{0} \varpi_{E} & { }^{\tau} \Delta^{-} \alpha^{-1} & I_{N}
\end{array}\right)\right) .
\end{aligned}
$$

Démonstration. Traitons d'abord rapidement les deux premiers cas, soit $i<t$, en travaillant de nouveau avec $M^{0}\left(s_{i}\right)$, sous-groupe de Levi de $G$ engendré par $M^{0}$ et $s_{i}$, et les radicaux unipotents respectifs $N^{0}\left(s_{i}\right)$ et $N^{0}\left(s_{i}\right)^{-}$de $P^{0}\left(s_{i}\right)=M^{0}\left(s_{i}\right) N^{0}$ et $P^{0}\left(s_{i}\right)^{-}=M^{0}\left(s_{i}\right) N^{0-}$. Pour $\Omega=\Omega(t, 2 t, k)$ on a (voir Corollaire 3 ) :

$$
\Omega s_{i} \Omega=\Omega s_{i}\left(\Omega \cap N^{0}\left(s_{i}\right)^{-}\right)\left(\Omega \cap N^{0}\left(s_{i}\right)\right)\left(\Omega \cap M^{0}\left(s_{i}\right)\right) .
$$

On vérifie immédiatement que $s_{i}\left(\Omega \cap N^{0}\left(s_{i}\right)^{ \pm}\right) s_{i}^{-1}$ est contenu dans $\Omega$ : la conjugaison par $s_{i}$ y permute des blocs identiques. Comme $s_{i}$ normalise $\Omega \cap M^{0}$ on aboutit à $\Omega s_{i} \Omega=\Omega s_{i}\left(\Omega \cap \operatorname{Im} j_{i}\right)$, d'où l'égalité cherchée si $t>i>0\left(\operatorname{car} \mathfrak{H}^{1} \subset \mathfrak{J}^{0}\right)$ et si $i=0\left(\operatorname{car} \Delta^{-\tau} \sigma \subset \Delta^{+} \alpha\right.$ par $\left.2.4(3)\right)$.

Dans le cas $i=t$ et $\Omega=\Omega(t, t, k)$ un calcul plus fin nous rendra service par la suite. Soit $\mathcal{P}$ le sous-groupe parabolique de $G$ conjugué du parabolique standard $\mathcal{P}^{\prime}$ de Levi $\iota(G L((t-1) N, F)) \times j_{0}\left(G_{1, k}\right)$ par $w=\iota\left(\left(\begin{array}{cc}0 & I_{(t-1) N} \\ I_{N} & 0\end{array}\right)\right)$, soit $\mathcal{N}$ son radical unipotent, soit $\mathcal{M}$ le sous-groupe de Levi de $G$ engendré par $M^{0}$ et les $s_{i}$ pour $1 \leqslant i \leqslant t-2$, enfin soit $\mathcal{N}^{-}$le radical unipotent du sous-groupe parabolique de $G$ opposé de $\mathcal{P}$ par rapport à $\mathcal{M}$. Alors $\Omega$ est décomposé par rapport à $\mathcal{P}$ (Corollaire $3)$ et on a :

$$
s_{t}\left(\Omega \cap \mathcal{N}^{-}\right) s_{t}^{-1}=\Omega \cap \mathcal{N}^{-} \quad ; \quad s_{t}(\Omega \cap \mathcal{N}) s_{t}^{-1}=\Omega \cap \mathcal{N} .
$$


Vérifions en effet la première égalité ; on calcule :

$$
s_{t}\left(\begin{array}{ccccc}
I_{N} & V_{1} & 0 & 0 & 0 \\
0 & I_{(t-1) N} & 0 & 0 & 0 \\
0 & V_{2} & I_{2 k} & 0 & 0 \\
V_{3}^{\prime} & T & V_{2}^{\prime} & I_{(t-1) N} & V_{1}^{\prime} \\
0 & V_{3} & 0 & 0 & I_{N}
\end{array}\right) s_{t}^{-1}=\left(\begin{array}{cccccc}
I_{N} & W_{1} & 0 & 0 & 0 \\
0 & I_{(t-1) N} & 0 & 0 & 0 \\
0 & V_{2} & I_{2 k} & 0 & 0 \\
W_{3}^{\prime} & T & V_{2}^{\prime} & I_{(t-1) N} & W_{1}^{\prime} \\
0 & W_{3} & 0 & 0 & I_{N}
\end{array}\right)
$$

avec $W_{1}=-{ }^{\tau} \sigma^{\tau} \varpi_{E}^{-1} V_{3}, W_{3}=\sigma^{-1} \varpi_{E} V_{1}, W_{3}^{\prime}=-V_{1}^{\prime \tau} \varpi_{E}^{\tau} \sigma^{-1}, W_{1}^{\prime}=V_{3}^{\prime} \varpi_{E}^{-1} \sigma$. Le bloc $V_{1}$ décrit $M_{1, t-1}\left(\mathfrak{J}^{0}\right)$. L'égalité est vérifiée si et seulement si le bloc $V_{3}$ décrit $M_{1, t-1}\left(\sigma^{-1} \varpi_{E} \mathfrak{J}^{0}\right)$ (cf. 2.1.c), ce qui est le cas dans $\Omega(t, r, k)$ si et seulement si $r=t$. La seconde égalité se vérifie de manière analogue.

On aboutit alors aisément à $\Omega s_{t} \Omega=\Omega s_{t}\left(\Omega \cap \operatorname{Im} j_{t}\right)=\Omega s_{t} j_{t}(\Omega(1,1, k))$. La partie $j_{t}\left(\Omega(1,1, k) \cap P_{1, k}\right)$ disparaît à gauche grâce à 2.4 (4) qui fournit l'inclusion ${ }^{\tau} \Delta^{+} \sigma^{-1} \varpi_{E} \subset \alpha^{-1} \Delta^{-}$et le résultat s'ensuit.

Lemme 4. Soit $w \in W_{t}$.

a) Si $0 \leqslant i \leqslant t-1$ et $l\left(s_{i} w\right)=1+l(w)$, on $a$ :

$$
\Omega(t, 2 t, k) s_{i} \Omega(t, 2 t, k) w \Omega(t, 2 t, k)=\Omega(t, 2 t, k) s_{i} w \Omega(t, 2 t, k) .
$$

b) $\operatorname{Sil} l\left(s_{t} w\right)=1+l(w)$, on $a$ :

$$
\Omega(t, t, k) s_{t} \Omega(t, t, k) w \Omega(t, t, k)=\Omega(t, t, k) s_{t} w \Omega(t, t, k) .
$$

Démonstration. Il suffit dans chaque cas de montrer que $w^{-1} j_{i}(\nabla) w$ est contenu dans le groupe $\Omega(t, r, k)$ considéré, où $j_{i}(\nabla)$ désigne le sous-groupe figurant à droite de l'égalité correspondante du lemme 3 . Or on peut réaliser $\mathbf{W}_{t}$ comme le groupe de Weyl affine d'un groupe symplectique $S p(2 t, K)$ sur un corps local non archimédien $K$ relativement au sous-groupe d'Iwahori standard

$$
\mathfrak{J}=\left(\begin{array}{cccc}
\mathfrak{o}_{K}^{\times} & \mathfrak{o}_{K} & \ldots & \mathfrak{o}_{K} \\
\mathfrak{p}_{K} & \ddots & & \vdots \\
\vdots & & \ddots & \mathfrak{o}_{K} \\
\mathfrak{p}_{K} & \cdots & \mathfrak{p}_{K} & \mathfrak{o}_{K}^{\times}
\end{array}\right) \cap S p(2 t, K),
$$

en envoyant les générateurs $\mathbf{s}_{i}$ de $\mathbf{W}_{t}$ sur les éléments $\mathrm{s}_{i}$ de $S p(2 t, K)$ définis comme les $s_{i}$ de 2.3 en remplaçant $N$ par $1, k$ par $0, \sigma$ par 1 et $\varpi_{E}$ par $\varpi_{K}$; on utilisera aussi les analogues $\mathrm{j}_{i}$ des plongements $j_{i}$ dans ce contexte. La condition de longueur s'exprime alors de la manière suivante $([\overline{\mathrm{BO}}], \mathrm{IV} .2 .4)$ :

$$
\begin{aligned}
l\left(\mathrm{~s}_{i} \mathrm{w}\right)=1+l(\mathrm{w}) & \Longleftrightarrow \mathfrak{J} \mathrm{s}_{i} \mathfrak{J} \mathrm{w} \mathfrak{J}=\mathfrak{J} \mathrm{s}_{i} \mathrm{w} \mathfrak{J} \\
& \Longleftrightarrow \begin{cases}\left.\mathrm{w}^{-1} \mathrm{j}_{i}\left(\begin{array}{cc}
1 & \mathfrak{o}_{K} \\
0 & 1
\end{array}\right)\right) \mathrm{w} \subset \mathfrak{J} \quad \text { si } i<t, \\
\left.\mathrm{w}^{-1} \mathrm{j}_{t}\left(\begin{array}{cc}
1 & 0 \\
\mathfrak{p}_{K} & 1
\end{array}\right)\right) \mathrm{w} \subset \mathfrak{J} \quad \text { si } i=t .\end{cases}
\end{aligned}
$$

Cette dernière condition peut s'exprimer à son tour en termes des valuations des entrées non nulles de w (qui appartiennent à $\pm \varpi_{K}^{\mathbb{Z}}$ ); sous cette forme elle implique l'inclusion $w^{-1} j_{i}(\nabla) w \subset \Omega$ cherchée, comme on le voit immédiatement pour $1 \leqslant$ $i \leqslant t-1$ et au prix d'une vérification plus soigneuse pour $i=0$ ou $t$.

Dans ce dernier cas par exemple, écrivons $\mathrm{w}=\mathrm{dw}_{0}$ avec $\mathrm{w}_{0} \in \mathrm{W}_{t}^{0}$ et $\mathrm{d}=$ $\operatorname{diag}\left(\varpi_{K}^{l_{1}}, \cdots, \varpi_{K}^{l_{t}}, \varpi_{K}^{-l_{t}}, \cdots \varpi_{K}^{-l_{1}}\right)$. Alors $l\left(\mathrm{~s}_{t} \mathrm{w}\right)=1+l(\mathrm{w})$ si et seulement si $l_{1} \geqslant 0$. De même $w$ s'écrit $d w_{0}$ dans $G_{t, 0}$ (plongé dans $G_{t, k}$ comme en 2.1.a). On examine l'image de $j_{t}\left(\left(\begin{array}{ccc}I_{N} & 0 & 0 \\ \Delta^{-} & I_{2 k} & 0 \\ \sigma^{-1} \mathfrak{H}^{1} \Delta^{-} \alpha^{-1} & I_{N}\end{array}\right)\right)$ dans la conjugaison $x \mapsto w^{-1} x w$. Le 
bloc $\Delta^{-}$d'origine (en $\left.(2,1)\right)$ va prendre une place sur la ligne médiane (ligne $\left.t+1\right)$ et être multiplié par $\varpi_{E}^{l_{1}}$ (à des produits par $\sigma$ près) ; il restera dans $\Omega$ pourvu que $l_{1} \geqslant 0$ (conditions (2) et (3) de 2.4).

2.6. Propagation d'inversibilité. Moyennant la proposition 4 (et donc l'hypothèse (H1)), un ingrédient essentiel de la démonstration du théorème 1 est le théorème de Bushnell et Kutzko cité en 1.1. Nous utiliserons tout particulièrement les homomorphismes injectifs d'algèbres à unité suivants.

a) Soit $G_{t-1, k}^{+}$l'ensemble des éléments de $G_{t-1, k}$ dont le plongement dans $L_{t, k}$ est $\left(Q_{t, k}, \Omega\right)$-positif, et soit $\mathcal{H}(t-1, r, k)^{+}$la sous-algèbre de $\mathcal{H}(t-1, r, k)$ formée des éléments de support contenu dans $\Omega(t-1, r, k) G_{t-1, k}^{+} \Omega(t-1, r, k)$. Comme $\mathcal{H}(t-1, r, k)$ est naturellement une sous-algèbre de $\mathcal{H}\left(L_{t, k}, \gamma \otimes \omega(t-1, r, k)\right) \simeq$ $\mathcal{H}(G L(N, F), \gamma) \otimes \mathcal{H}(t-1, r, k)$ (plongement via $f \mapsto \mathcal{I} \otimes f)$, on obtient par restriction de $\mathcal{T}_{L_{t, k}}^{+}$:

$$
\mathcal{R}_{t-1}^{+}: \mathcal{H}(t-1, r, k)^{+} \underset{\text { alg }}{\hookrightarrow} \mathcal{H}(t, r, k)
$$

qui vérifie $\operatorname{Supp} \mathcal{R}_{t-1}^{+}(f)=\Omega(t, r, k) \operatorname{Supp} f \Omega(t, r, k)$ et dont l'image est la sousalgèbre des fonctions de support contenu dans $\Omega(t, r, k) G_{t-1, k}^{+} \Omega(t, r, k)$.

b) Soit $G L(t N, F)^{+}$l'ensemble des éléments de $G L(t N, F)$ dont le plongement dans $M_{t, k}$ via $\iota$ est $\left(P_{t, k}, \Omega\right)$-positif, et soit $\mathcal{A}(t, t)^{+}$la sous-algèbre de $\mathcal{A}(t, t)(1.4)$ formée des éléments de support contenu dans $\Gamma(t, t) G L(t N, F)^{+} \Gamma(t, t)$. Comme $\mathcal{A}(t, t)$ est naturellement une sous-algèbre de $\mathcal{H}\left(M_{t, k}, \gamma(t, t) \otimes \delta\right)$, isomorphe à $\mathcal{H}(G L(t N, F), \gamma(t, t)) \otimes \mathcal{H}(S p(2 k, F), \delta)$, on obtient par restriction de $\mathcal{T}_{M_{t, k}}^{+}$:

$$
\mathcal{T}_{\mathcal{A}}^{+}: \mathcal{A}(t, t)^{+} \underset{\mathrm{alg}}{\hookrightarrow} \mathcal{H}(t, r, k)
$$

qui vérifie $\operatorname{Supp} \mathcal{T}_{\mathcal{A}}^{+}(f)=\Omega(t, r, k) \iota(\operatorname{Supp} f) \Omega(t, r, k)$ et dont l'image est la sousalgèbre des fonctions de support contenu dans $\Omega(t, r, k) \iota\left(G L(t N, F)^{+}\right) \Omega(t, r, k)$.

Remarque. On a renoncé à faire apparaître dans les notations le fait que les ensembles d'éléments positifs $G_{t-1, k}^{+}$et $G L(t N, F)^{+}$dépendent de $\Omega=\Omega(t, r, k)$ c'est-àdire de $r$, comme on va le voir immédiatement.

Lemme 5. Supposons $r=2 t$.

(i) La sous-algèbre $\mathcal{A}(t, t)^{+}$est formée des fonctions de support contenu dans $\Gamma(t, t) \tilde{W}\left(\mathfrak{B}_{t}\right)^{+} \Gamma(t, t)$, où $\tilde{W}\left(\mathfrak{B}_{t}\right)^{+}$est le semi-groupe produit de $W_{0}\left(\mathfrak{B}_{t}\right)$ par $D\left(\mathfrak{B}_{t}\right)^{+}=\left\{\operatorname{diag}\left(\varpi_{E}^{l_{1}} I_{N}, \cdots, \varpi_{E}^{l_{t}} I_{N}\right) / l_{1}, \ldots, l_{t} \in \mathbb{N}\right\}$.

(ii) Les éléments de $W_{t-1}^{0} \subset G_{t-1, k}$ sont $\left(Q_{t, k}, \Omega(t, 2 t, k)\right)$-positifs.

Démonstration. Il s'agit de vérifier que les éléments de $\iota\left(\tilde{W}\left(\mathfrak{B}_{t}\right)^{+}\right) \subset M_{t, k}$ sont précisément les éléments $\left(P_{t, k}, \Omega(t, 2 t, k)\right)$-positifs de $\iota\left(\tilde{W}\left(\mathfrak{B}_{t}\right)\right)$. Or le choix de $r=2 t$ assure précisément que toute permutation $\iota(w), w \in W_{0}\left(\mathfrak{B}_{t}\right)$, normalise $\Omega(t, 2 t, k) \cap N_{t, k}$ et $\Omega(t, 2 t, k) \cap N_{t, k}^{-}$, puisque tous les blocs $N \times N$ de $\Gamma^{+}(t, 2 t) \sigma$ sont identiques, et de même pour $\sigma^{-1} \Gamma^{-}(t, 2 t)$. L'action des éléments de $\iota\left(D\left(\mathfrak{B}_{t}\right)\right)$ est facile à examiner.

(ii) Là aussi l'hypothèse $r=2 t$ est cruciale. Le bloc $(1,2)$ de $\Omega(t, 2 t, k)$ par exemple (dans une écriture par blocs subordonnée à la décomposition (2) de $V$ dans 2.1.a), a la forme $\left[\begin{array}{lllllll}\mathfrak{J}^{0} & \ldots & \mathfrak{J}^{0} & \Delta^{+} & \mathfrak{J}^{0} \sigma & \ldots & \mathfrak{J}^{0} \sigma\end{array}\right]$, d'où sa stabilité par action à droite de $W_{t-1}^{0}$. 
Lemme 6. Sous l'hypothèse (H2), l'élément $E_{s(t, k)}^{(2 t)}$ de l'algèbre $\mathcal{H}(t, 2 t, k)$ est inversible.

Démonstration. L'inversibilité de $E_{s(1, k)}^{(2)}$ dans $\mathcal{H}(1,2, k)$ résulte de $(\mathrm{H} 2)$ puisque le coefficient $b_{s}$ est non nul. Soit $t \geqslant 2$. L'élément $s(t, k)$ est conjugué de $s_{0}$ par $h$, où $h=s_{t-1} s_{t-2} \ldots s_{1}$. Posons $\Omega=\Omega(t, 2 t, k)$. On a :

$$
\Omega h \Omega s_{0} \Omega h^{-1} \Omega=\Omega h s_{0} h^{-1} \Omega=\Omega s(t, k) \Omega .
$$

En effet, comme tous les générateurs considérés appartiennent à $S_{t}^{0}$, il suffit grâce au lemme 4 de vérifier que la longueur de $h s_{0} h^{-1}$ est $(t-1)+1+(t-1)$ (on a, dans les notations de la démonstration du lemme $\left.4:[\mathfrak{J s}(t, k) \mathfrak{J}: \mathfrak{J}]=q_{K}^{2 t-1}\right)$.

Il découle du lemme 2 et de $(*)$ que l'on a dans $\mathcal{H}(t, 2 t, k)$ :

$$
E_{s(t, k)}^{(2 t)} \equiv E_{h} E_{s_{0}}^{(2 t)} E_{h^{-1}}
$$

pourvu que ce produit soit non nul. Comme $h$ appartient à $\iota\left(W_{0}\left(\mathfrak{B}_{t}\right)\right)$ on sait par (b) et le lemme 5 (i) que $E_{h}$ est l'image par $\mathcal{T}_{\mathcal{A}}^{+}$d'un élément $a_{h^{\prime}}$ de $\mathcal{A}(t, t)^{+}$ de support $\Gamma(t, t) h^{\prime} \Gamma(t, t)$, avec $h=\iota\left(h^{\prime}\right)$. De la structure de $\mathcal{A}(t, t)$ (Proposition 2 et BK1 5.4.6) on déduit que $a_{h^{\prime}}$ est inversible dans $\mathcal{A}(t, t)^{+}$(car son inverse est combinaison linéaire d'éléments de support contenu dans $\left.\Gamma(t, t) W_{0}\left(\mathfrak{B}_{t}\right) \Gamma(t, t)\right)$. Alors $E_{h}$ est inversible dans $\mathcal{H}(t, 2 t, k)$, d'inverse $\mathcal{T}_{\mathcal{A}}^{+}\left(a_{h^{\prime}}^{-1}\right)$. De même $E_{h^{-1}}$ est inversible dans $\mathcal{H}(t, 2 t, k)$.

Enfin l'hypothèse (H2) assure que l'élément $E_{s(1, k)}^{(2)}$ de $\mathcal{H}(1,2, k)$ a pour inverse une combinaison linéaire de lui-même et de $\mathcal{I}$. Ses images successives par les homomorphismes $\mathcal{R}_{j}^{+}$gardent la même propriété ((a) et Lemme 5 (ii)) donc $E_{s_{0}}$ est inversible dans $\mathcal{H}(t, 2 t, k)$. Finalement $E_{s(t, k)}^{(2 t)}$ est inversible comme produit d'éléments inversibles.

Lemme 7. Sous l'hypothèse (H3), l'élément $E_{q(t, k)}^{(t)}$ de l'algèbre $\mathcal{H}(t, t, k)$ peut être normalisé de façon à vérifier la relation quadratique

$$
\left(E_{q(t, k)}^{(t)}\right)^{2}=a_{q} E_{q(t, k)}^{(t)}+b_{q} \mathcal{I}
$$

Démonstration. Reprenons le sous-groupe parabolique $\mathcal{P}=\mathcal{M N}$ utilisé dans la démonstration du lemme 3: on y a établi (5) que $q(t, k)=s_{t}$ est $(\mathcal{P}, \Omega)$-positif. On utilise alors l'homomorphisme d'algèbres de 1.1:

$$
\mathcal{T}_{\mathcal{M}}^{+}: \mathcal{H}\left(\mathcal{M}, \omega_{\mid \Omega \cap \mathcal{M}}\right)^{+} \hookrightarrow \mathcal{H}(t, t, k) .
$$

Le sous-groupe de Levi $\mathcal{M}$ est naturellement isomorphe à $G L((t-1) N, F) \times G_{1, k}$, de sorte que l'algèbre $\mathcal{H}\left(\mathcal{M}, \omega_{\mid \Omega \cap \mathcal{M}}\right)$ s'identifie à $\mathcal{A}(t-1, t-1) \otimes \mathcal{H}(1,1, k)$, dans laquelle $e_{q(1, k)}^{(1)}$ vérifie la relation $\left(e_{q(1, k)}^{(1)}\right)^{2}=a_{q} e_{q(1, k)}^{(1)}+b_{q} \mathcal{I}$. L'image $q(t, k)$ de $q(1, k)$ dans $\mathcal{M}$ par l'isomorphisme ci-dessus appartient à $\mathcal{M}^{+}$, donc l'image de $e_{q(1, k)}^{(1)}$ par $\mathcal{T}_{\mathcal{M}}^{+}$, qui est un élément de support $\Omega q(t, k) \Omega$, vérifie la même relation, c.q.f.d.

2.7. Isomorphismes d'algèbres de Hecke. Nous avons prouvé l'inversibilité de $E_{s(t, k)}^{(2 t)}$ dans $\mathcal{H}(t, 2 t, k)$ et celle de $E_{q(t, k)}^{(t)}$ dans $\mathcal{H}(t, t, k)$ (lemmes 6 et 7 ). Pour exploiter ces résultats, ainsi que ceux des lemmes 3 et 4 , il faut pouvoir passer d'une algèbre à l'autre: la proposition qui suit est le point-clé des démonstrations des théorèmes 1 et 2. 
Proposition 5. Pour tout $r$ tel que $t \leqslant r \leqslant 2 t-1$ il y a un isomorphisme d'algèbres canonique

$$
\mathcal{E}_{r}: \mathcal{H}(t, r, k) \stackrel{\simeq}{\longrightarrow} \mathcal{H}(t, r+1, k)
$$

qui vérifie, pour tout $w \in W_{t}: \mathcal{E}_{r}\left(E_{w}^{(r)}\right) \equiv E_{w}^{(r+1)}$.

Plus précisément, soit $\Lambda(t, r, k)$ le sous-groupe engendré par $\Omega(t, r, k)$ et $\Omega(t, r+1, k)$. Pour tout $w \in W_{t}$, le sous-espace de $\mathcal{H}(t, r, k)$ formé des fonctions de support contenu dans $\Lambda(t, r, k) w \Lambda(t, r, k)$ est de dimension 1.

Démonstration. L'idée de base est celle de la démonstration de la proposition 2 (1.4), mais cette fois on a moins de renseignements sur les algèbres considérées, la mise en œuvre est donc plus élaborée. Noter qu'en niveau 0 la proposition est trivialement vérifiée puisque les groupes $\Omega(t, r, k)$ sont indépendants de $r$; on se place donc en niveau strictement positif.

1ère étape: description du sous-groupe $\Lambda(t, r, k)$. Montrons que:

(i) le produit $\Lambda(t, r, k)=\left(\Omega(t, r+1, k) \cap N^{-}\right)(\Omega \cap M)(\Omega(t, r, k) \cap N)$ est un groupe, engendré par $\Omega(t, r, k)$ et $\Omega(t, r+1, k)$.

On a $\Omega(t, r, k)=\left(\Omega(t, r, k) \cap N^{-}\right)(\Omega(t, r, k) \cap M)(\Omega(t, r, k) \cap N)$ et

$$
\left\{\begin{array}{c}
\Omega(t, r, k) \cap M=\Omega(t, r+1, k) \cap M, \\
\Omega(t, r, k) \cap N^{-} \subset \Omega(t, r+1, k) \cap N^{-}, \\
\Omega(t, r, k) \cap N \supset \Omega(t, r+1, k) \cap N .
\end{array}\right.
$$

On remarque que $\Omega(t, r+1, k) \cap N^{-}=\mathcal{Z}^{-}\left(\Omega(t, r, k) \cap N^{-}\right)$et $\Omega(t, r, k) \cap N=$ $\mathcal{Z}(\Omega(t, r+1, k) \cap N)$ où $\mathcal{Z}^{-}$et $\mathcal{Z}$ sont les sous-groupes centraux de $N^{-}$et $N$ suivants :

$$
\begin{aligned}
\mathcal{Z}^{-} & =\left\{\left(\begin{array}{ccc}
I_{t N} & 0 & 0 \\
0 & I_{2 k} & 0 \\
Y & 0 & I_{t N}
\end{array}\right) \in G_{t, k} / Y \in \sigma^{-1} \Gamma^{-}(t, r+1) \text { et } Y_{i j}=0 \text { si } i-j \neq r-t\right\}, \\
\mathcal{Z} & =\left\{\left(\begin{array}{ccc}
I_{t N} & 0 & C \\
0 & I_{2 k} & 0 \\
0 & 0 & I_{t N}
\end{array}\right) \in G_{t, k} / C \in \Gamma^{+}(t, r) \sigma \text { et } C_{i j}=0 \text { si } j-i \neq r-t\right\}
\end{aligned}
$$

(les indices $i$ et $j$ s'entendent ici relativement à une décomposition de $M_{t N}(F)$ en blocs $N \times N)$. Autrement dit, on isole dans $\sigma^{-1} \Gamma^{-}(t, r+1)$ la sous-diagonale qui diffère de $\sigma^{-1} \Gamma^{-}(t, r)$ (blocs dans $\left.\sigma^{-1} \mathfrak{H}^{1}\right)$, et on isole dans $\Gamma^{+}(t, r) \sigma$ la sousdiagonale qui diffère de $\Gamma^{+}(t, r+1) \sigma$ (blocs dans $\varpi_{E}^{-1} \mathfrak{H}^{1} \sigma$ ).

Il s'agit de voir que le produit $\mathcal{Z}^{-} \Omega(t, r, k)$ est un groupe, i.e. de vérifier que $\Omega(t, r, k) \mathcal{Z}^{-} \subset \mathcal{Z}^{-} \Omega(t, r, k)$, ou encore $(\Omega(t, r, k) \cap N) \mathcal{Z}^{-} \subset \mathcal{Z}^{-} \Omega(t, r, k)$. Mais le produit $(\Omega(t, r, k) \cap N) \mathcal{Z}^{-}=\mathcal{Z}(\Omega(t, r+1, k) \cap N) \mathcal{Z}^{-}$est contenu dans $\mathcal{Z} \Omega(t, r+$ $1, k)$, lui-même contenu dans $\mathcal{Z Z}^{-} \Omega(t, r, k)$; on n'a plus qu'à montrer que $\mathcal{Z Z}^{-} \subset$ $\mathcal{Z}^{-} \Omega(t, r, k)$. Cette fois le calcul a lieu dans $G_{t, 0}$, dans lequel (i) a été établi en 1.4 (démonstration de la proposition 2), c.q.f.d.

2ème étape: première réduction.

La deuxième assertion de la proposition entraîne la première. En effet son application à $w=1$ montre que la représentation $\operatorname{Ind}_{\Omega(t, r, k)}^{\Lambda(t, r, k)} \omega(t, r, k)$ est irréductible. Comme $\Lambda(t, r, k)=\Omega(t, r+1, k) \Omega(t, r, k)$ (une seule double classe), on a:

$$
\begin{aligned}
& \operatorname{Hom}_{\Lambda(t, r, k)}\left(\operatorname{Ind}_{\Omega(t, r, k)}^{\Lambda(t, r, k)} \omega(t, r, k), \operatorname{Ind}_{\Omega(t, r+1, k)}^{\Lambda(t, r, k)} \omega(t, r+1, k)\right) \\
& \simeq \operatorname{Hom}_{\Omega(t, r, k)}\left(\omega(t, r, k),\left.\left(\operatorname{Ind}_{\Omega(t, r+1, k)}^{\Lambda(t, r)} \omega(t, r+1, k)\right)\right|_{\Omega(t, r, k)}\right) \\
& \simeq \operatorname{Hom}_{\Omega(t, r, k)}\left(\omega(t, r, k), \operatorname{Ind}_{\Omega(t, r, k) \cap \Omega(t, r+1, k)}^{\Omega(t, r, k)}\left(\left.\omega(t, r+1, k)\right|_{\Omega(t, r, k) \cap \Omega(t, r+1, k)}\right)\right) .
\end{aligned}
$$


Ainsi $\operatorname{Ind}_{\Omega(t, r, k)}^{\Lambda(t, r, k)} \omega(t, r, k)$ est une sous-représentation de $\operatorname{Ind}_{\Omega(t, r+1, k)}^{\Lambda(t, r, k)} \omega(t, r+1, k)$ qui a même dimension : elles sont irréductibles et isomorphes. L'isomorphisme $\mathcal{E}_{r}$ cherché provient alors directement de [BK1, 4.1.3] par composition :

$$
\begin{aligned}
\mathcal{H}(t, r, k) & \stackrel{\simeq}{\longrightarrow} \mathcal{H}\left(G_{t, k}, \operatorname{Ind}_{\Omega(t, r, k)}^{\Lambda(t, r, k)} \omega(t, r, k)\right) \\
& \simeq \mathcal{\simeq}\left(G_{t, k}, \operatorname{Ind}_{\Omega(t, r+1, k)}^{\Lambda(t, r, k)} \omega(t, r+1, k)\right) \stackrel{\simeq}{\longrightarrow} \mathcal{H}(t, r+1, k) .
\end{aligned}
$$

Un entrelacement entre les deux représentations induites est unique à scalaire près donc détermine de manière unique le deuxième isomorphisme, qui préserve évidemment le support. L'assertion sur l'image de $E_{w}^{(r)}$ est alors conséquence directe de [BK1, 4.1.5] et du lemme 2.

Reste à montrer que :

(ii) La seule $\Omega(t, r, k)$-double classe de $\Lambda(t, r, k) w \Lambda(t, r, k)$ qui entrelace $\omega(t, r, k)$ est $\Omega(t, r, k) w \Omega(t, r, k)$.

3ème étape : réduction à $G_{t, 0}$.

Comme $\Lambda(t, r, k) w \Lambda(t, r, k)=\Omega(t, r, k) \mathcal{Z}^{-} w \mathcal{Z}^{-} \Omega(t, r, k)$, (ii) équivaut à montrer que

(iii) $z \in \mathcal{Z}^{-} w \mathcal{Z}^{-}$et $z$ entrelace $\omega(t, r, k) \Longrightarrow z \in \Omega(t, r, k) w \Omega(t, r, k)$.

Mais $\mathcal{Z}^{-} w \mathcal{Z}^{-}$est contenu dans $G_{t, 0}$ qui commute à $S p(2 k, F)$ (i.e. $\left.G_{0, k}\right)$ dans $G_{t, k}:$ si $z$ entrelace $\omega(t, r, k)$, il entrelace sa restriction $\omega(t, r, 0) \otimes \delta$ à

$$
\Omega(t, r, k) \cap\left(G_{t, 0} \times S p(2 k, F)\right) \simeq \Omega(t, r, 0) \times \Delta .
$$

Il entrelace donc $\omega(t, r, 0)$, et si (iii) est établi pour $k=0$ on déduit

$$
z \in \Omega(t, r, 0) w \Omega(t, r, 0) \subset \Omega(t, r, k) w \Omega(t, r, k), \text { c.q.f.d. }
$$

4ème étape: réduction au cas où $r=t$.

On suppose maintenant $k=0$; par 2.1.d : $\Omega(t, r, 0)=\Gamma(2 t, r)^{\Sigma_{t}} \cap G_{t, 0}$. On reprend alors le procédé du corollaire 1 et de la proposition 2 : on identifie $G_{t, 0}$ au sous-groupe de $G_{r, 0}$ image du plongement

$$
g \longmapsto\left(\begin{array}{ccc}
I_{(r-t) N} & 0 & 0 \\
0 & g & 0 \\
0 & 0 & I_{(r-t) N}
\end{array}\right) \quad\left(g \in G_{t, 0}\right),
$$

de sorte que $\Omega(t, r+1,0)=\Omega(r, r+1,0) \cap G_{t, 0}$ et $\Omega(t, r, 0)=\Omega(r, r, 0) \cap G_{t, 0}$. Travaillons dans $G_{r, 0}$ par rapport au sous-groupe parabolique $\mathcal{P}$ formé de matrices triangulaires supérieures par blocs, avec un facteur de Levi $\mathcal{M}$ diagonal par blocs et isomorphe à $G L(N, F) \times \ldots G L(N, F) \times G_{t, 0}(r-t$ facteurs $G L(N, F)$, le facteur $G_{t, 0}$ étant l'image du plongement ci-dessus).

Comme la paire $(\Omega(r, r, 0), \omega(r, r, 0))$ est décomposée par rapport à $\mathcal{P}$, si un élément $z \in \mathcal{Z}^{-} w \mathcal{Z}^{-} \subset G_{t, 0}$ entrelace $\omega(t, r, 0)$ dans $G_{t, 0}$, il entrelace $\gamma \otimes \cdots \otimes$ $\gamma \otimes \omega(t, r, 0)$ dans $\mathcal{M}$, donc il entrelace $\omega(r, r, 0)$ dans $G_{r, 0}$. Si donc (iii) est prouvé pour $r=t$, on conclut que $z \in G_{t, 0} \cap \Omega(r, r, 0) w \Omega(r, r, 0)$. Or soient $a$ et $b$ dans $\Omega(r, r, 0)$ tels que $z a=b w$; en écrivant cette égalité avec des matrices $(3,3)$ par blocs comme dans le plongement de $G_{t, 0}$ ci-dessus, et vu la forme des éléments $z$ et $w$ de $G_{t, 0}$, on voit qu'alors $z a_{22}=b_{22} w$, donc $z$ appartient à $\Omega(t, r, 0) w \Omega(t, r, 0)$, c.q.f.d.

5ème étape: cas de $\Omega(t, t, 0)$. 
Travaillons avec le parabolique de Siegel $P=M N$ de $G_{t, 0}$. On a (2.1.d et 1.3)

$$
\Omega(t, t, 0)=J_{P}\left(\beta, \mathfrak{A}_{2 t}\right)^{\Sigma_{t}} \cap G_{t, 0}, \quad \Omega(t, t+1,0)=J_{P^{-}}\left(\beta, \mathfrak{A}_{2 t}\right)^{\Sigma_{t}} \cap G_{t, 0}
$$

et

$$
\Lambda(t, t, 0)=J\left(\beta, \mathfrak{A}_{2 t}\right)^{\Sigma_{t}} \cap G_{t, 0} .
$$

Modifions provisoirement les notations pour faciliter la comparaison avec Bl3]. Notons $\bar{X}$ l'intersection de $X \subset G L(2 t N, F)$ avec $G_{t, 0}=S p(2 t N, F)$ et notons $H^{1}=H^{1}\left(\beta, \mathfrak{A}_{2 t}\right)^{\Sigma_{t}}, J^{1}=J^{1}\left(\beta, \mathfrak{A}_{2 t}\right)^{\Sigma_{t}}$ et $J=J\left(\beta, \mathfrak{A}_{2 t}\right)^{\Sigma_{t}}$, de sorte que $\Omega(t, t, 0)=$ $\bar{J}_{P}$ et $\Lambda(t, t, 0)=\bar{J}$. On peut alors reproduire le diagramme de loc. cit. $\$ 4.2$ en remplaçant respectivement $\theta_{0}, \eta_{0}$ et $\kappa_{0}$ par $\theta_{0}(t, t), \eta_{0}(t, t)$ et $\kappa_{0}(t, t)$ (Corollaire 2). Les arguments (et les conventions) de loc. cit., Proposition 4.2, s'appliquent de la même façon pour établir que :

1) $\omega(t, t, 0)$ est produit tensoriel de $i\left(\widetilde{\sigma_{0}(t, t)}\right)$ par $i\left(\widetilde{\kappa_{0}(t, t)}\right)$ (où $\left(\bar{J}_{P}, i\left(\widetilde{\sigma_{0}(t, t)}\right)\right)$ et $\left(\bar{J}, i\left(\widetilde{\sigma_{0}(t, t)}\right)\right)$ sont des paires décomposées au-dessus de $\left.\left(\Gamma^{\times t}, \sigma_{0}^{\otimes t}\right)\right)$;

2) la restriction de $\operatorname{Ind}_{\bar{J}_{P}}^{\bar{J}} i\left(\widetilde{\kappa_{0}(t, t)}\right)$ à $\bar{J}^{1}$ est isomorphe à $\operatorname{Ind}_{\bar{J}_{P}^{1}}^{\bar{J}^{1}} i\left(\widetilde{\eta_{0}(t, t)}\right)$ qui est une représentation irréductible ;

$3)$ pour tout $w \in W_{t}$, l'espace d'entrelacements $I_{w}\left(\operatorname{Ind}_{\bar{J}_{P}^{1}}^{\bar{J}^{1}} i\left(\widetilde{\eta_{0}(t, t)}\right)\right)$ est de dimension 1 (on a remarqué en 2.3 que $W_{t}$ est ici contenu dans $B^{\times}$).

On veut en déduire (ii), autrement dit (Lemme 2 et [BK1], 4.1.5) que l'espace d'entrelacements $I_{w}\left(\operatorname{Ind}_{\bar{J}_{P}}^{\bar{J}} \omega(t, t, 0)\right)$ est lui-même de dimension 1. Or la représentation $\operatorname{Ind}_{\bar{J}_{P}}^{\bar{J}} \omega(t, t, 0)$ est équivalente à $i\left(\widetilde{\sigma_{0}(t, t)}\right) \otimes \operatorname{Ind}_{\bar{J}_{P}}^{\bar{J}} i\left(\widetilde{\kappa_{0}(t, t)}\right)$. Comme dans [Bl3], on reprend donc la démonstration de [BK1], Proposition 5.3.2 ; les trois propriétés ci-dessus permettent d'exprimer un élément non nul de $I_{w}\left(\operatorname{Ind}_{\bar{J}_{P}}^{\bar{J}} \omega(t, t, 0)\right)$ sous la forme $X \otimes Y$ où $Y \neq 0$ appartient à $I_{w}\left(\operatorname{Ind}_{\bar{J}_{P}^{1}}^{\bar{J}^{1}} i\left(\widetilde{\eta_{0}(t, t)}\right)\right)$ et $X \neq 0$ est un endomorphisme de l'espace de $i\left(\widetilde{\sigma_{0}(t, t)}\right)$. On a pour tout $x \in \bar{J} \cap(\bar{J})^{w}$ :

$$
\begin{aligned}
\left(X \circ i\left(\widetilde{\sigma_{0}(t, t)}\right)(x)\right) & \otimes\left(Y \circ \operatorname{Ind}_{\bar{J}_{P}}^{\bar{J}} i\left(\widetilde{\kappa_{0}(t, t)}\right)(x)\right) \\
= & \left(i\left(\widetilde{\sigma_{0}(t, t)}\right)\left(w x w^{-1}\right) \circ X\right) \otimes\left(\operatorname{Ind}_{\bar{J}_{P}}^{\bar{J}} i\left(\widetilde{\kappa_{0}(t, t)}\right)\left(w x w^{-1}\right) \circ Y\right)
\end{aligned}
$$

d'où l'existence d'un scalaire $\alpha(x)$ tel que

$$
\left\{\begin{array}{l}
X \circ i\left(\widetilde{\sigma_{0}(t, t)}\right)(x)=\alpha(x) i\left(\widetilde{\sigma_{0}(t, t)}\right)\left(w x w^{-1}\right) \circ X, \\
Y \circ \operatorname{Ind}_{\bar{J}_{P}}^{\bar{J}} i\left(\widetilde{\kappa_{0}(t, t)}\right)(x)=\alpha(x)^{-1} \operatorname{Ind}_{\bar{J}_{P}}^{\bar{J}} i\left(\widetilde{\kappa_{0}(t, t)}\right)\left(w x w^{-1}\right) \circ Y .
\end{array}\right.
$$

On vérifie aisément que $\alpha$ est un caractère de $\bar{J} \cap(\bar{J})^{w}$, entièrement déterminé par la seconde condition puisque $Y$ est unique à scalaire près. Or, pour $w \in$ $W_{t}$, l'intersection $\bar{J} \cap(\bar{J})^{w}$ contient $\bar{J} \cap M^{0} \simeq \Gamma^{\times t}$ : l'opérateur $X$ entrelace les représentations irréductibles $\iota\left(\sigma_{0}^{\otimes t}\right)$ et $\iota\left(\sigma_{0}^{\otimes t}\right)^{w} \otimes \alpha$ de $\Gamma^{\times t}$, il est donc unique à scalaire près, c.q.f.d.

2.8. Conclusion de la démonstration. Notre objectif est le théorème 1: les paires décomposées $(\Omega(t, r, k), \omega(t, r, k))$ de la proposition 4 sont des paires couvrantes. Nous allons montrer que pour tout $t \geq 1$, et sous les hypothèses (H1), (H2) et (H3), $(\Omega(t, r, k), \omega(t, r, k))$ est une paire couvrante de $(\Gamma \times \Omega(t-1, r, k), \gamma \otimes \omega(t-$ 
$1, r, k))$ relativement à $Q_{t, k}$ - on convient de poser $(\Omega(0, r, k), \omega(0, r, k))=(\Delta, \delta)$. Par transitivité (1.1), le théorème en découle par récurrence sur $t$.

Le lemme 6 affirme que $E_{s(t, k)}^{(2 t)}$ est inversible dans l'algèbre $\mathcal{H}(t, 2 t, k)$, le lemme 7 que $E_{q(t, k)}^{(t)}$ est inversible dans l'algèbre $\mathcal{H}(t, t, k)$ (car $\left.b_{q} \neq 0\right)$. La proposition 5 implique alors que les éléments $E_{s(t, k)}^{(r)}$ et $E_{q(t, k)}^{(r)}$ sont inversibles dans $\mathcal{H}(t, r, k)$ pour tout $r$.

$$
\begin{gathered}
\text { Notons } \Omega=\Omega(t, r, k) \text { et posons } z=\left(\begin{array}{ccc}
\varpi_{E} & 0 & 0 \\
0 & I_{2(t-1) N+2 k} & 0 \\
0 & 0 & \varpi_{E}^{-1}
\end{array}\right) . \text { On a : } \\
z=s(t, k) q(t, k) \text { et } \quad \Omega s(t, k) \Omega q(t, k) \Omega=\Omega z \Omega .
\end{gathered}
$$

(Les relations (3) et (4) de 2.4 permettent de vérifier que :

$$
s(t, k)\left(\Omega \cap Q^{-}\right) s(t, k)^{-1} \subset \Omega \cap Q \quad \text { et } \quad q(t, k)(\Omega \cap Q) q(t, k)^{-1} \subset \Omega \cap Q^{-} .
$$

Alternative : utiliser le lemme 4 et la proposition 5.)

Il en résulte (Lemme 2) que $E_{z}^{(r)} \equiv E_{s(t, k)}^{(r)} E_{q(t, k)}^{(r)}$ dans $\mathcal{H}(t, r, k)$, donc $E_{z}^{(r)}$ est inversible. Or $z$ est un élément $\left(Q_{t, k}, \Omega\right)$-positif de $L_{t, k}$ dont la puissance $e$-ième ( $e=$ $e(E / F))$ appartient à $c\left(\Omega \cap L_{t, k}\right)$ avec $c=\operatorname{diag}\left(\varpi_{F} I_{N}, I_{2(t-1) N+2 k}, \varpi_{F}^{-1} I_{N}\right)$, élément fortement $\left(Q_{t, k}, \Omega\right)$-positif du centre de $L_{t, k}$. Les doubles classes $\Omega z \Omega$ et $\Omega c \Omega$ supportent un sous-espace de dimension 1 de $\mathcal{H}(t, r, k)$ et la relation $\left(\Gamma \varpi_{E} \Gamma\right)^{e}=$ $\Gamma \varpi_{F} \Gamma$ entraîne (par positivité de $z$ et $c$ et grâce à l'homomorphisme $\mathcal{T}_{L_{t, k}}^{+}$de 1.1) : $E_{z}^{e} \equiv E_{c}$ dans $\mathcal{H}(t, r, k)$. L'élément $E_{c}$ est donc inversible, ce qui équivaut au fait que $(\Omega, \omega)$ soit couvrante relativement à $Q_{t, k}(1.1)$.

\section{Structure Des AlgÈbres De Hecke}

Nous décrivons dans ce paragraphe les algèbres de Hecke des paires couvrantes obtenues au théorème 1 ; on y conserve donc les hypothèses de ce théorème et les notations du paragraphe 2 (en particulier celles de 2.3 concernant $W_{t}$ ). On définit des éléments remarquables de nos algèbres $\mathcal{H}(t, r, k)$ qui vérifient les relations (quadratiques et de tresse) nécessaires pour engendrer une sous-algèbre qui est de convolution sur le groupe de Coxeter $\left(\mathbf{W}_{t}, \mathbf{S}_{t}\right)$ de type $\tilde{C}_{t}$ et dont les paramètres sont entièrement déterminés par le cas $t=1$ (3.1 et 3.2). On montre alors, moyennant une hypothèse supplémentaire, que cette sous-algèbre est $\mathcal{H}(t, r, k)$ tout entière (3.3). On termine par quelques exemples (3.4).

3.1. Algèbres de convolution sur $\left(\mathbf{W}_{t}, \mathbf{S}_{t}\right)$. Si t est au moins égal à 2 , l'ensemble de générateurs $\mathbf{S}_{t}$ de $\mathbf{W}_{t}$ rencontre exactement trois classes de conjugaison de $\mathbf{W}_{t}$, en $\left\{\mathbf{s}_{0}\right\},\left\{\mathbf{s}_{1}, \ldots, \mathbf{s}_{t-1}\right\}$ et $\left\{\mathbf{s}_{t}\right\}$ ([Bo] IV.1.3-variante immédiate si $\left.t=1\right)$. Une algèbre de convolution (complexe) sur $\left(\mathbf{W}_{t}, \mathbf{S}_{t}\right)$ est donc donnée par trois paires de paramètres, $\left(a_{0}, b_{0}\right),\left(a_{1}, b_{1}\right)$ (pour $\left.1 \leqslant i \leqslant t-1\right)$ et $\left(a_{t}, b_{t}\right)$. C'est la $\mathbb{C}$-algèbre $\mathbf{H}\left(\mathbf{W}_{t}, \mathbf{S}_{t}\right)$ admettant la présentation par générateurs et relations suivante (voir par exemple [Bo, exercice IV.2.23) :

$$
\begin{array}{ll}
\text { générateurs : } & \mathbf{e}_{\mathbf{s}_{i}} \quad(0 \leqslant i \leqslant t) ; \\
\text { relations } & \left\{\begin{array}{l}
\mathbf{e}_{\mathbf{s}_{0}}^{2}=a_{0} \mathbf{e}_{\mathbf{s}_{0}}+b_{0} \mathcal{I} ; \\
\text { quadratiques }
\end{array}\right. \\
\mathbf{e}_{\mathbf{s}_{i}}^{2}=a_{1} \mathbf{e}_{\mathbf{s}_{i}}+b_{1} \mathcal{I} \quad \text { pour } 1 \leqslant i \leqslant t-1 \\
\mathbf{e}_{\mathbf{s}_{t}}^{2}=a_{t} \mathbf{e}_{\mathbf{s}_{t}}+b_{t} \mathcal{I} ;
\end{array}
$$




$$
\begin{aligned}
& \text { relations } \\
& \text { de tresse }
\end{aligned}\left\{\begin{array}{l}
\mathbf{e}_{\mathbf{s}_{i}} \mathbf{e}_{\mathbf{s}_{j}}=\mathbf{e}_{\mathbf{s}_{j}} \mathbf{e}_{\mathbf{s}_{i}} \quad \text { si }|j-i| \geqslant 2 ; \\
\mathbf{e}_{\mathbf{s}_{0}} \mathbf{e}_{\mathbf{s}_{1}} \mathbf{e}_{\mathbf{s}_{0}} \mathbf{e}_{\mathbf{s}_{1}}=\mathbf{e}_{\mathbf{s}_{1}} \mathbf{e}_{\mathbf{s}_{0}} \mathbf{e}_{\mathbf{s}_{1}} \mathbf{e}_{\mathbf{s}_{0}} \quad \text { si } t \geqslant 2 ; \\
\mathbf{e}_{\mathbf{s}_{i}} \mathbf{e}_{\mathbf{s}_{i+1}} \mathbf{e}_{\mathbf{s}_{i}}=\mathbf{e}_{\mathbf{s}_{i+1}} \mathbf{e}_{\mathbf{s}_{i}} \mathbf{e}_{\mathbf{s}_{i+1}} \quad \text { si } 1 \leqslant i \leqslant t-2 ; \\
\mathbf{e}_{\mathbf{s}_{t}} \mathbf{e}_{\mathbf{s}_{t-1}} \mathbf{e}_{\mathbf{s}_{t}} \mathbf{e}_{\mathbf{s}_{t-1}}=\mathbf{e}_{\mathbf{s}_{t-1}} \mathbf{e}_{\mathbf{s}_{t}} \mathbf{e}_{\mathbf{s}_{t-1}} \mathbf{e}_{\mathbf{s}_{t}} \quad \text { si } t \geqslant 2 .
\end{array}\right.
$$

Elle a pour base comme espace vectoriel complexe la famille $\left(\mathbf{e}_{\mathbf{w}}\right)_{\mathbf{w} \in \mathbf{W}_{t}}$ d'éléments $\mathbf{e}_{\mathbf{w}}=\mathbf{e}_{\mathbf{s}_{i_{1}}} \cdots \mathbf{e}_{\mathbf{s}_{i_{v}}}$, où $\mathbf{w}=\mathbf{s}_{i_{1}} \cdots \mathbf{s}_{i_{v}}$ est une décomposition réduite de $\mathbf{w}$ dans $\left(\mathbf{W}_{t}, \mathbf{S}_{t}\right)$ - l'élément $\mathbf{e}_{\mathbf{w}}$ ne dépend pas de la décomposition réduite de $\mathbf{w}$ choisie pour le définir. Si $b_{0}, b_{1}$ et $b_{t}$ sont non nuls, les éléments $\mathbf{e}_{\mathbf{w}}$ sont tous inversibles.

Proposition 6. On se place dans les hypothèses (H1), (H2) et (H3) de 2.2 et on note $\mathcal{H}=\mathcal{H}\left(G_{t, k}, \omega(t, r, k)\right)$ et $\Omega=\Omega(t, r, k)$.

1. (i) Il existe un élément $e_{s_{0}}$ de $\mathcal{H}$, de support $\Omega s_{0} \Omega$, qui vérifie la relation quadratique $e_{s_{0}}^{2}=a_{s} e_{s_{0}}+b_{s} \mathcal{I}$.

(ii) Soit $1 \leqslant i \leqslant t-1$. Il existe un élément $e_{s_{i}}$ de $\mathcal{H}$, de support $\Omega s_{i} \Omega$, qui vérifie la relation quadratique $e_{s_{i}}^{2}=\left(q_{E}^{f}-1\right) e_{s_{i}}+q_{E}^{f} \mathcal{I}$ (avec $f=N /[E: F]$, cf. 1.2).

(iii) Il existe un élément $e_{s_{t}}$ de $\mathcal{H}$, de support $\Omega s_{t} \Omega$, qui vérifie la relation quadratique $e_{s_{t}}^{2}=a_{q} e_{s_{t}}+b_{q} \mathcal{I}$.

2. Les éléments $e_{s_{0}}, \ldots, e_{s_{t}}$ vérifient les relations de tresse ci-dessus.

3. L'unique homomorphisme d'algèbres de $\mathbf{H}\left(\mathbf{W}_{t}, \mathbf{S}_{t}\right)$ dans $\mathcal{H}$ envoyant $\mathbf{e}_{\mathbf{s}_{i}}$ sur $e_{s_{i}}$ pour $0 \leqslant i \leqslant t$ envoie $\mathbf{e}_{\mathbf{w}}, \mathbf{w} \in \mathbf{W}_{t}$, sur un élément $e_{w}$ de support $\Omega w \Omega$.

4. Cet homomorphisme est injectif et a pour image la sous-algèbre $\mathcal{H}_{W}$ de $\mathcal{H}$ formée des éléments de support contenu dans $\Omega W_{t} \Omega$. En particulier $\mathcal{H}_{W}$ admet pour base la famille des $e_{w}, w \in W_{t}$, qui sont inversibles dans $\mathcal{H}_{W}$.

Démonstration. Grâce à la proposition 5, on peut, pour établir 1, choisir dans chaque cas la valeur de $r$ la plus commode. Pour $r=t$ l'existence de $e_{s_{t}}$ est donnée par le lemme 7, d'où (iii). Pour (ii) il suffit d'exhiber les $e_{s_{i}}$ pour $r=2 t$. Or soit $\psi_{i}$ l'élément de $G L(t N, F)$ tel que $\iota\left(\psi_{i}\right)=s_{i}$; il existe un élément $x_{i}$ de $\mathcal{A}(t, t)$, de support $\Gamma(t, t) \psi_{i} \Gamma(t, t)$, qui vérifie la relation quadratique voulue (Proposition 2 et BK1 5.4.6, 5.6.6) : son image par $\mathcal{T}_{\mathcal{A}}^{+}$convient (Lemme 5 et 2.6 (b)). Enfin pour (i) et $r=2 t$, on prend l'image de $e_{s(1, k)} \in \mathcal{H}(1,2, k)$ par le composé des homomorphismes $\mathcal{R}_{j}^{+}$(Lemme 5 et 2.6 (a)).

Montrons à présent que :

$$
w, x \in W_{t} \text { et } l(w x)=l(w)+l(x) \quad \Longrightarrow \quad E_{w}^{(r)} E_{x}^{(r)} \equiv E_{w x}^{(r)}
$$

Si pour une paire $(w, x)$, ceci est vrai pour une valeur de $r$, c'est vrai pour toute valeur de $r$ (Proposition 5). On se ramène - récurrence sur $l(w)$ - à démontrer l'assertion pour $w \in S_{t}$. Elle découle alors du lemme 4.

Admettons pour l'instant les relations de tresse qui seront établies au paragraphe suivant et vérifions la fin de l'énoncé. Le fait que le support de $e_{w}$ soit $\Omega w \Omega$ et son inversibilité sont conséquences de $(*)$ et des définitions, d'où 3 . Pour avoir injectivité de l'homomorphisme ainsi construit, il suffit de montrer que les doubles classes $\Omega w \Omega, w \in W_{t}$, sont disjointes. Si $k=0$ cela provient du fait que $\Omega(t, r, 0)=\Gamma(2 t, r)^{\Sigma} \cap S p(2 t N, F)$ : les doubles classes $\Gamma(2 t, r) w \Gamma(2 t, r)$ pour $w \in \tilde{W}\left(\mathfrak{B}_{2 t}\right)$ sont deux à deux disjointes (Proposition 2 ), or $W_{t}$ correspond à $\operatorname{diag}\left(\Gamma, \ldots, \Gamma,{ }^{\tau} \Gamma, \ldots,{ }^{\tau} \Gamma\right)$ près à un sous-système de $\tilde{W}\left(\mathfrak{B}_{2 t}\right)^{\Sigma}$. Dans le cas général, 
soient $w$ et $x$ deux éléments de $W_{t}$ tels que $\Omega w \Omega=\Omega x \Omega$ et soient $a$ et $b$ dans $\Omega$ tels que $x a=b w$. Ecrivons cette égalité comme un produit de matrices $3 \times 3$ par blocs, de sorte que les éléments de $G_{t, 0}$, vus dans $G_{t, k}$ via l'inclusion naturelle de 2.1.a, ont pour deuxième ligne $\left(0, I_{2 k}, 0\right)$ et pour deuxième colonne ${ }^{t}\left(0, I_{2 k}, 0\right)$; il en est donc ainsi pour $x$ et $w$. On obtient alors une égalité semblable dans $G_{t, 0}$ : $x a^{\prime}=b^{\prime} w$, où $a^{\prime}$ et $b^{\prime}$ s'obtiennent à partir de $a$ et $b$ en gardant les blocs $(1,1),(1,3)$, $(3,1)$ et $(3,3)$. Comme $a$ et $b$ appartiennent à $\Omega$, les éléments $a^{\prime}$ et $b^{\prime}$ appartiennent à $\Gamma(2 t, r)^{\Sigma}$, donc $x=w$, c.q.f.d.

3.2. Les relations de tresse. Reste à établir les relations de tresse. De nouveau, grâce à la proposition 5 , on pourra choisir pour chaque relation la valeur de $r$ la plus commode. Rappelons un fait très général (démonstration immédiate) ; soient $x$ et $w$ deux éléments de $W_{t}$ de carré 1 (modulo $\Omega$ ), alors :

$(\diamond) \quad x^{-1} \Omega x \cap \Omega w \Omega w^{-1} \subset \Omega \Longrightarrow\left\{\begin{array}{l}E_{x} E_{w}(x w)=\operatorname{vol}(\Omega) E_{x}(x) E_{w}(w), \\ E_{w} E_{x}(w x)=\operatorname{vol}(\Omega) E_{w}(w) E_{x}(x) .\end{array}\right.$

- Relations de commutation : $e_{s_{i}} e_{s_{j}}=e_{s_{j}} e_{s_{i}}$ si $|j-i| \geqslant 2$.

Si $j<t$ et $i \geqslant 1$ la relation de commutation analogue est valide dans $\mathcal{A}(t, t)$, elle se transporte donc par $\mathcal{T}_{\mathcal{A}}^{+}$si $r=2 t$ (Proposition 2 et 2.6).

Si $j<t$ et $i=0$ (donc $t \geqslant 3$ ) on se place dans le sous-groupe de Levi $\mathcal{M}_{j}$ engendré par $M^{0}, s_{0}$ et $s_{j}$; ses blocs diagonaux sont $G L(N, F)(t-3$ facteurs), $G L(2 N, F)$ et $G_{1, k}$. Pour $r=2 t$, les éléments $s_{0}$ et $s_{j}$ sont $\left(\mathcal{M}_{j} P^{0}, \Omega\right)$-positifs donc $e_{s_{0}}$ et $e_{s_{j}}$ sont les images par $\mathcal{T}_{\mathcal{M}}^{+}$d'éléments qui commutent.

Si $j=t$, donc $i \leqslant t-2$, les éléments $e_{s_{i}} e_{s_{t}}$ et $e_{s_{t}} e_{s_{i}}$ ont même support $\Omega s_{i} s_{t} \Omega$ par $(*)$, il suffit de vérifier qu'ils ont même valeur en $s_{i} s_{t}$. Vu $(\diamond)$ ci-dessus, on commence par étudier l'intersection $s_{i}^{-1} \Omega s_{i} \cap \Omega s_{t} \Omega s_{t}^{-1}$. Pour cela, on utilise la décomposition de $\Omega$ par rapport à $Q_{t, k}=L_{t, k} R_{t, k}$ et on note $\Omega^{-}=\Omega \cap R_{t, k}^{-}$, $\Omega_{L}=\Omega \cap L_{t, k}, \Omega^{+}=\Omega \cap R_{t, k}^{+}$. Comme $s_{i}$ appartient à $L$ on a

$$
s_{i}^{-1} \Omega s_{i}=\left(s_{i}^{-1} \Omega^{-} s_{i}\right)\left(s_{i}^{-1} \Omega_{L} s_{i}\right)\left(s_{i}^{-1} \Omega^{+} s_{i}\right) .
$$

D'autre part la conjugaison par $s_{t}$ échange $R$ et $R^{-}$et stabilise $L$, et on vérifie que $s_{t} \Omega^{+} s_{t}^{-1} \subset \Omega^{-}$, d'où

$$
\Omega s_{t} \Omega s_{t}^{-1}=\Omega^{-} \Omega_{L}\left(s_{t} \Omega^{-} s_{t}^{-1}\right) .
$$

L'intersection cherchée s'effectue donc terme à terme (dans $R^{-}$, dans $L$, dans $R$ ) et comme $s_{i}^{-1} \Omega^{-} s_{i}$ est contenu dans $\Omega^{-}$si $r=2 t$, l'intersection est contenue dans $\Omega$ si $r=2 t$.

Il n'y a plus qu'à vérifier que les opérateurs d'entrelacement $e_{s_{i}}\left(s_{i}\right)$ et $e_{s_{t}}\left(s_{t}\right)$ commutent. Or soit $X_{\gamma}$ l'espace de la représentation $\gamma$, soit $X_{\delta}$ celui de $\delta$ et soit

$$
X=\bigotimes_{i=1}^{t} X_{\gamma} \otimes X_{\delta}
$$

L'opérateur $e_{s_{t}}\left(s_{t}\right)$ ne travaille que sur la première composante $X_{\gamma}$, tandis que $e_{s_{0}}\left(s_{0}\right)$ travaille sur la dernière et que $e_{s_{i}}\left(s_{i}\right)$ pour $1 \leqslant i \leqslant t-1$ échange les composantes $X_{\gamma}$ de rang $t-i+1$ et $t-i$ : il ne touche pas à la première composante si $i<t-1$, d'où le résultat.

- Relation $e_{s_{i}} e_{s_{i+1}} e_{s_{i}}=e_{s_{i+1}} e_{s_{i}} e_{s_{i+1}}$ si $1 \leqslant i \leqslant t-2$.

Elle est valide dans $\mathcal{A}(t, t)$ et se transporte par $\mathcal{T}_{\mathcal{A}}^{+}$pour $r=2 t$ (Proposition 2 et 2.6). 
- Relation $e_{s_{0}} e_{s_{1}} e_{s_{0}} e_{s_{1}}=e_{s_{1}} e_{s_{0}} e_{s_{1}} e_{s_{0}}$ si $t \geqslant 2$.

On peut supposer $r=2 t$ puis travailler dans $G_{2, k}$ : la relation se transportera ensuite par les homomorphismes $\mathcal{R}_{j}^{+}$successifs (2.6 (a) et Lemme 5). L'élément $x=s_{1} s_{0} s_{1}=s(2, k)$ est de longueur 3 et l'on sait par $(*)$ que le support de $e_{s_{1}} e_{s_{0}} e_{s_{1}}$ est $\Omega x \Omega$; il s'agit donc de montrer que $e_{s_{0}}$ et $E_{x}$ commutent. Les produits $e_{s_{0}} E_{x}$ et $E_{x} e_{s_{0}}$ ont même support $\Omega s_{0} x \Omega$ par $(*)$. Les opérateurs $e_{s_{0}}\left(s_{0}\right)$ et $E_{x}(x)$ commutent évidemment: le premier ne travaille que sur la deuxième composante $X_{\gamma}$ de (†) et le second sur la première. D'après $(\diamond)$ il reste à vérifier que $x^{-1} \Omega x \cap \Omega s_{0} \Omega s_{0}^{-1} \subset \Omega$, ce que l'on fait comme plus haut (cas $j=t$ ) en remarquant que $x^{-1} \Omega^{-} x \subset \Omega^{+}$et $s_{0} \Omega^{-} s_{0}^{-1} \subset \Omega^{-}$.

- Relation $e_{s_{t-1}} e_{s_{t}} e_{s_{t-1}} e_{s_{t}}=e_{s_{t}} e_{s_{t-1}} e_{s_{t}} e_{s_{t-1}}$ si $t \geqslant 2$.

Comme précédemment, posant $w=s_{t} s_{t-1} s_{t}$ il suffit de montrer que $e_{s_{t-1}} E_{w}$ et $E_{w} e_{s_{t-1}}$ ont même valeur en $w s_{t-1}$. On travaille cette fois par rapport à $\mathcal{P}=\mathcal{M} N^{0}$ de facteur de Levi $\mathcal{M} \simeq G L(2 N, F) \times G_{t-2, k}$ engendré par $M^{0}, s_{t-1}$ et les $s_{i}$ pour $i \leqslant t-3$ et de radical unipotent $\mathcal{N}$. En effet $s_{t-1}$ appartient à $\mathcal{M}$ et

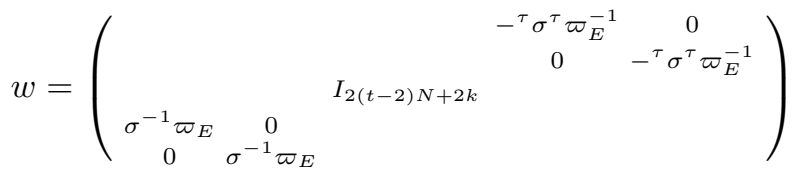

normalise $\mathcal{M}$. En outre $w$ normalise $\Omega \cap \mathcal{M}$ et vérifie $w \Omega \cap \mathcal{N} w^{-1} \subset \Omega \cap \mathcal{N}^{-}$. L'intersection $s_{t-1}^{-1} \Omega s_{t-1} \cap \Omega w \Omega w^{-1}$ s'effectue donc terme à terme : dans $\mathcal{N}^{-}$, dans $\mathcal{M}$, dans $\mathcal{N}$; comme $s_{t-1}^{-1} \Omega \cap \mathcal{N} s_{t-1}$ est contenu dans $\Omega \cap \mathcal{N}$ si $r=2 t$, l'intersection est contenue dans $\Omega$ si $r=2 t$. Enfin, les opérateurs $e_{s_{t-1}}\left(s_{t-1}\right)$ et $E_{w}(w)$ commutent: tous deux ne travaillent que sur les deux premières composantes $X_{\gamma}$ de $X, e_{s_{t-1}}\left(s_{t-1}\right)$ par $v_{1} \otimes v_{2} \mapsto v_{2} \otimes v_{1}$ et $E_{w}(w)$ par $v_{1} \otimes v_{2} \mapsto \Phi\left(v_{2}\right) \otimes \Phi\left(v_{1}\right)$, où $\Phi$ entrelace $\gamma$ et $\left(\gamma^{\sigma^{-1} \varpi_{E}}\right)^{*}$.

3.3. Présentation de Bernstein. La proposition 6 décrit entièrement la sousalgèbre $\mathcal{H}_{W}$ de $\mathcal{H}$ formée des éléments de support contenu dans $\Omega W_{t} \Omega$. Pour pouvoir montrer que cette sous-algèbre est en fait $\mathcal{H}$ tout entière il nous faut une hypothèse supplémentaire (voir la proposition 3) :

Hypothèse (H4). La représentation induite $\rho=\operatorname{Ind}_{\Delta}^{S p(2 k, F)} \delta$ est irréductible (donc supercuspidale).

Sous cette hypothèse, si $\pi$ est une représentation irréductible supercuspidale autoduale de $G L(N, F)$ contenant le type simple maximal $(\Gamma, \gamma)$, la paire $\left(\Gamma^{\times t} \times\right.$ $\Delta, \gamma^{\otimes t} \otimes \delta$ ) est un type pour la classe d'inertie de la représentation supercuspidale irréductible $\pi^{\otimes t} \otimes \rho$ de $G L(N, F)^{t} \times S p(2 k, F) \simeq M_{t, k}^{0}=M^{0}$. Comme $(\Omega, \omega)$ est une paire couvrante de la précédente, la paire $(\Omega, \omega)$ est elle-même un type pour la classe d'inertie $\left[M^{0}, \pi^{\otimes t} \otimes \rho\right]_{G}$ dans $G=G_{t, k}$ ([BK2], Théorème 8.3).

Puisque la représentation $\pi$ est autoduale, le normalisateur $N_{G}\left(M^{0}\right)$ de $M^{0}$ dans $G$ fixe la classe d'équivalence de $\pi^{\otimes t} \otimes \rho$ donc aussi la classe d'inertie $\left[M^{0}, \pi^{\otimes t} \otimes \rho\right]_{M^{0}}$. Le quotient $\mathbf{W}_{t}^{0}=N_{G}\left(M^{0}\right) / M^{0}$ a été décrit en 2.3. L'hypothèse (H4) nous permet d'utiliser : 
Théorème (Bushnell-Kutzko [BK3]). Soit $K$ un sous-groupe compact de $G$ contenant $\Omega$. On définit un homomorphisme (d'espaces vectoriels) injectif par

$$
\begin{array}{rll}
\mathcal{H}\left(M^{0}, \gamma^{\otimes t} \otimes \delta\right) \otimes_{\mathbb{C}} \mathcal{H}(K, \omega) & \stackrel{\mathcal{T}}{\longrightarrow} & \mathcal{H}\left(G_{t, k}, \omega\right) \\
\varphi \otimes f & \longmapsto & \mathbb{T}_{M^{0}}^{+}(\varphi) \star f .
\end{array}
$$

La dimension de $\mathcal{H}(K, \omega)$ est au plus égale au cardinal de $\mathbf{W}_{t}^{0}$; s'il y a égalité, l'homomorphisme $\mathcal{T}$ est un isomorphisme.

Ce théorème va nous fournir la surjectivité de l'homomorphisme d'algèbres de la proposition 6 : il suffit de montrer que $\mathbb{T}_{M^{0}}^{+}\left(\mathcal{H}\left(M^{0}, \gamma^{\otimes t} \otimes \delta\right)\right)$ est contenue dans la sous-algèbre $\mathcal{H}_{W}$ de $\mathcal{H}$, puis de trouver un sous-groupe $K$ tel que $\mathcal{H}(K, \omega)$ soit contenue dans $\mathcal{H}_{W}$ et de dimension $\left|W_{t}^{0}\right|$.

Le premier point est facile, puisque $\mathbb{T}_{M^{0}}^{+}$est l'unique prolongement de

$$
\mathcal{T}_{M^{0}}^{+}: \mathcal{H}\left(M^{0}, \gamma^{\otimes t} \otimes \delta\right)^{+} \underset{\text { alg }}{\hookrightarrow} \mathcal{H}(G, \omega)
$$

en un homomorphisme d'algèbres $\mathcal{H}\left(M^{0}, \gamma^{\otimes t} \otimes \delta\right) \underset{\text { alg }}{\hookrightarrow} \mathcal{H}(G, \omega)$ (1.1). Son image est engendrée par celle de $\mathcal{T}_{M^{0}}^{+}$et par l'inverse d'un élément de support $\Omega z \Omega$ où $z$ est un élément fortement positif du centre de $M^{0}$ ([BK2]). Or l'image de $\mathcal{T}_{M^{0}}^{+}$est engendrée comme espace vectoriel par des éléments de support $\Omega x \Omega$ où $x$ appartient à $M^{0}$ et entrelace $\gamma^{\otimes t} \otimes \delta$ : un tel $x$ peut être choisi dans $W_{t}$ (Proposition 3) donc ces éléments appartiennent à $\mathcal{H}_{W}$ et y sont inversibles, c.q.f.d.

Passons au choix de $K$. Le sous-groupe $\Gamma$ fixe la chaîne de réseaux $\left(L_{0, i}\right)_{i \in \mathbb{Z}}$ de $F^{N}$ associée à l'ordre $\mathfrak{A}_{0}(1.2)$; la chaîne de réseaux de $F^{t N}$ associée à l'ordre $\mathfrak{A}_{t}$ contient $L_{0}(t)=\bigoplus_{j=1}^{t} L_{0,0}$. L'espace symplectique ambiant $V(t, k)$ (2.1.a) est somme directe orthogonale de $V_{-t} \oplus V_{t}$ et de $V_{0}$. Pour tout réseau $\Lambda$ de $V_{0}$, la somme $L_{0}(t) \oplus \Lambda \oplus L_{0}(t)$ est un réseau de $V(t, k)$ (le premier $L_{0}(t)$ est plongé dans $V_{-t}$, le second dans $\left.V_{t}\right)$. Fixons alors le réseau $\Lambda=\mathfrak{o}_{F}\left[\alpha^{\tau} \Delta^{+} L_{0}(1)\right]$. On vérifie aisément (à l'aide des propriétés de 2.4) que pour tout $t \geqslant 1$ et tout $r \geqslant t$, le réseau somme $L_{0}(t) \oplus \Lambda \oplus L_{0}(t)$ est fixé par $\Omega(t, r, k)$. Or ce réseau est aussi fixé par les éléments $s_{i}$ pour $0 \leqslant i \leqslant t-1$.

Soit alors $K$ le fixateur dans $G_{t, k}$ du réseau $L_{0}(t) \oplus \Lambda \oplus L_{0}(t)$ : il est compact et contient $W_{t}^{0}$ et $\Omega(t, r, k)$. L'algèbre $\mathcal{H}(K, \omega)$ est de dimension au plus égale à $\left|W_{t}^{0}\right|$ par le théorème de Bushnell-Kutzko, au moins égale à $\left|W_{t}^{0}\right|$ par la proposition 6 . On a donc égalité et $\mathcal{H}(K, \omega)$ est contenue dans $\mathcal{H}_{W}$. Nous venons de montrer :

Théorème 2. Sous les hypothèses (H1), (H2), (H3) (2.2) et (H4) (3.3), l'homomorphisme d'algèbres de la proposition 6 est un isomorphisme: l'algèbre de Hecke $\mathcal{H}\left(G_{t, k}, \omega(t, r, k)\right)$ relative à la paire couvrante $(\Omega(t, r, k), \omega(t, r, k))$ du théorème 1 est une algèbre de convolution sur $\left(\mathbf{W}_{t}, \mathbf{S}_{t}\right)$, de paramètres $\left(a_{0}, b_{0}\right)=\left(a_{s}, b_{s}\right)$, $\left(a_{1}, b_{1}\right)=\left(q_{E}^{f}-1, q_{E}^{f}\right)$ et $\left(a_{t}, b_{t}\right)=\left(a_{q}, b_{q}\right)$.

Corollaire 4. La structure de l'algèbre $\mathcal{H}\left(G_{t, k}, \omega(t, r, k)\right)$ est entièrement déterminée par les paramètres $q_{E}^{f}$, attaché à $(\Gamma, \gamma)$, et $a_{s}, b_{s}, a_{q}, b_{q}$, attachés à la paire couvrante "de hauteur 1 " $(\Omega(1,1, k), \omega(1,1, k))$ (ou $(\Omega(1,2, k), \omega(1,2, k)))$ dans $S p(2 N+2 k, F)$.

Remarques. 1) Les théorèmes 1 et 2 sont valides pour $t=1$ : sous les hypothèses (H1) à (H3) les paires $(\Omega(1,1, k), \omega(1,1, k))$ et $(\Omega(1,2, k), \omega(1,2, k))$ sont couvrantes, 
et moyennant (H4) leur algèbre de Hecke est une algèbre de convolution sur $\left(\mathbf{W}_{1}, \mathbf{S}_{1}\right)$, comme on pouvait s'y attendre.

2) Le cas où les types $(\Gamma, \gamma)$ et $(\Delta, \delta)$ sont tous deux de niveau 0 a déjà été traité par Morris ([Mo], où l'on trouve de plus des précisions sur les paramètres).

\subsection{Quelques exemples.}

3.4.a. Le cas de $S p(4, F)$.

On trouve dans $\mathrm{BB}$ la liste des paires couvrantes dans $S p(4, F)$ correspondant

- aux classes d'inertie $[G L(2, F), \pi]_{S p(4, F)}$ des représentations supercuspidales autoduales $\pi$ du sous-groupe de Levi de Siegel $G L(2, F)$ (soit $N=2$ et $k=0$ ) ;

- aux classes d'inertie $\left[F^{\times} \times S L(2, F), \chi \otimes \rho\right]_{S p(4, F)}$ des produits tensoriels d'un caractère $\chi$ de $F^{\times}$de carré non ramifié par une représentation supercuspidale $\rho$ de $S L(2, F)($ soit $N=1$ et $k=1)$;

ainsi que les paramètres des algèbres de Hecke associées.

Ces paires couvrantes vérifient les hypothèses $(\mathrm{H} 1)$ à $(\mathrm{H} 4)$ (c'est immédiat dans le cas $N=1$; pour $N=2$ la vérification est plus facile dans les notations de 3.4.b ci-dessous), les théorèmes 1 et 2 fournissent donc des paires couvrantes

- attachées aux classes d'inertie $\left[G L(2, F)^{\times t}, \pi^{\otimes t}\right]_{S p(4 t, F)}$ dans $S p(4 t, F)$;

- attachées aux classes d'inertie $\left[G L(1, F)^{\times t} \times S p(2, F), \chi^{\otimes t} \otimes \rho\right]_{S p(2 t+2, F)}$ dans $S p(2 t+2, F)$;

ainsi que la structure des algèbres de Hecke associées.

3.4.b. Le cas de $S p(2 t N, F)$.

Un résultat essentiel de $\mathrm{Bl3}$ ] est précisément que, dans les notations de 2.1.c, les groupes

$$
\Omega(1,1,0)=\left(\begin{array}{cc}
\Gamma & \varpi_{E}^{-1} \mathfrak{H}^{1} \sigma \\
\sigma^{-1} \mathfrak{J}^{0} \varpi_{E} & { }_{\Gamma}
\end{array}\right) \cap S p(2 N, F)
$$

et

$$
\Omega(1,2,0)=\left(\begin{array}{cc}
\Gamma & \mathfrak{J}^{0} \sigma \\
\sigma^{-1} \mathfrak{H}^{1} & \tau \\
& \tau
\end{array}\right) \cap S p(2 N, F)
$$

portent des paires couvrantes de $(\Gamma, \gamma)$ relativement au parabolique de Siegel. Les hypothèses (H2) et (H3) sont vérifiées (loc. cit. §3.3). Le théorème 1 s'applique donc pour fournir des paires couvrantes dans $\operatorname{Sp}(2 t N, F)$ attachées aux classes d'inertie $\left[G L(N, F)^{\times t}, \pi^{\otimes t}\right]_{S p(2 t N, F)}$, où $\pi$ est une représentation supercuspidale autoduale de $G L(N, F)$ contenant $(\Gamma, \gamma)$. La structure des algèbres de Hecke correspondantes est décrite par le théorème 2 , mais cette fois les paramètres $\left(a_{s}, b_{s}\right)$ et $\left(a_{q}, b_{q}\right)$ du cas $t=1$ ne sont pas connus en général.

3.4.c. Un exemple dans $S p(2 t N+N, F)(N>1)$.

L'entier $N$ est pair. Partons d'une strate simple gauche $\left[\mathfrak{A}_{0}, n_{0}, 0, \beta\right]([\underline{\mathrm{St}}])$ dans un espace symplectique $V$ de dimension $N$ sur $F$, avec $n_{0}>0$ et telle que $E=F[\beta]$ soit une extension de degré $N$ de $F$. Alors $E_{0}=F\left[\beta^{2}\right]$ est fixé par l'anti-involution de $\operatorname{End}_{F}(V)$ et, pour tout vecteur non nul $v$ de $V$, l'espace est somme directe de $E_{0} v$ et $\beta E_{0} v$, sous-espaces totalement isotropes maximaux. En se plaçant dans une base symplectique adaptée à une telle décomposition, on se ramène à une strate simple $\left[\mathfrak{A}_{0}, n_{0}, 0, \beta\right]$ dans $M_{N}(F)$, gauche relativement à la structure symplectique 
standard de matrice $w_{N} \alpha$, avec $\alpha=\left(\begin{array}{cc}I_{N / 2} & 0 \\ 0 & -I_{N / 2}\end{array}\right)$. L'élément $\sigma=\left(\begin{array}{cc}I_{N / 2} & 0 \\ 0 & -I_{N / 2}\end{array}\right)$ vérifie alors toutes les propriétés de 2.1.c : on poursuit avec $\alpha=\sigma$.

Pour tout sous-groupe $H$ de $G L_{N}(F)$, notons $\bar{H}$ son intersection avec $S p(N, F)$ (relativement à la structure ci-dessus). D'après [St], Théorème 5.2, tout caractère simple gauche $\theta_{0} \in \mathcal{C}\left(\mathfrak{A}_{0}, 0, \beta\right)$ (gauche signifie précisément que $\theta_{0}$ est fixé par $x \mapsto$ $\sigma^{\tau} x^{-1} \sigma^{-1}$ qui est ici une involution) permet de construire des représentations supercuspidales de $S p(N, F)$ comme suit. Soit $\eta_{0}$ l'unique représentation irréductible de $\bar{J}^{1}\left(\beta, \mathfrak{A}_{0}\right)$ qui contient la restriction à $\bar{H}^{1}\left(\beta, \mathfrak{A}_{0}\right)$ de $\theta_{0}$. Pour tout prolongement $\delta$ de $\eta_{0}$ à $\bar{J}\left(\beta, \mathfrak{A}_{0}\right)=\Delta$, la représentation $\rho=\operatorname{Ind}_{\Delta}^{S p(N, F)} \delta$ est supercuspidale irréductible: la paire $(\Delta, \delta)$ vérifie $(\mathrm{H} 4)$.

Par ailleurs le même caractère simple gauche $\theta_{0} \in \mathcal{C}\left(\mathfrak{A}_{0}, 0, \beta\right)$ permet de construire des représentations supercuspidales autoduales de $G L(N, F)$, qu'on préfère ici attacher à la strate simple $\left[\mathfrak{A}_{0}, n_{0}, 0,2 \beta\right]$ et au caractère simple $\theta_{0}^{2} \in \mathcal{C}\left(\mathfrak{A}_{0}, 0,2 \beta\right)$ (voir Bl3] Lemme 4.3.1). En effet, soit $\eta_{0}^{(2)}$ l'unique représentation irréductible de $J^{1}\left(\beta, \mathfrak{A}_{0}\right)$ qui contient $\theta_{0}^{2}$ et soit $\kappa$ une $\beta$-extension de $\eta_{0}^{(2)}$ à $J\left(\beta, \mathfrak{A}_{0}\right)$. Comme $\theta_{0}^{2}$ est gauche, $x \mapsto \kappa\left(\sigma^{\tau} x^{-1} \sigma^{-1}\right)$ est une autre $\beta$-extension de $\eta_{0}^{(2)}$, donc équivalente à $x \mapsto \kappa(x) \chi(x)$ pour un caractère $\chi$ de $J / J^{1} \simeq k_{E}^{\times}$(BK1] Théorème 5.2.2). Or $x \mapsto \sigma^{\tau} x \sigma^{-1}$ réalise la conjugaison $x \mapsto \bar{x}$ de $E$ sur $E_{0}$ : en itérant on obtient $\chi(x / \bar{x})=1$ pour tout $x \in k_{E}^{\times}$.

Si $E$ est non ramifiée sur $E_{0}$, il existe un caractère $\chi^{\prime}$ de $k_{E_{0}}^{\times}$tel que $\chi=$ $\chi^{\prime} \circ N_{E / E_{0}}$. Prolongeons-le en un caractère $\chi^{0}$ de $k_{E}^{\times}$.

Si $E$ est ramifiée sur $E_{0}$, la comparaison des déterminants de $\kappa$ et $\kappa \chi$ fournit $\chi(-1)=1$, donc $\chi$ est un carré, soit $\chi=\left(\chi^{0}\right)^{2}$ pour un caractère $\chi^{0}$ de $k_{E}^{\times}$.

Dans les deux cas ci-dessus, $\kappa^{\prime}=\kappa \circ \chi^{0}$ est une $\beta$-extension autoduale de $\eta_{0}^{(2)}$ et $(\Gamma, \gamma)=\left(J\left(\beta, \mathfrak{A}_{0}\right), \kappa^{\prime}\right)$ est un type simple de Bushnell-Kutzko figurant dans une représentation supercuspidale autoduale $\pi$ de $G L(N, F)$ et possédant les propriétés de 2.1.c.

Partons donc de notre caractère simple gauche $\theta_{0} \in \mathcal{C}\left(\mathfrak{A}_{0}, 0, \beta\right)$ et considérons dans $G L(3 N, F)$ les sous-groupes $H^{1}(3,2)$ et $H^{1}(3,3)$ de 1.3 , corollaire 2 : ils portent des paires décomposées au-dessus de $\left(H^{1} \times H^{1} \times H^{1}, \theta_{0} \otimes \theta_{0} \otimes \theta_{0}\right)$. Par conjugaison par $\operatorname{diag}\left(I_{N}, I_{N}, \sigma\right)$ on obtient des paires décomposées au-dessus de $\left(H^{1} \times H^{1} \times{ }^{\tau} H^{1}, \theta_{0} \otimes \theta_{0} \otimes \theta_{0}^{\sigma}\right)$. On en déduit comme en [Bl3], 1.2, que pour toutes paires $(\Delta, \delta)$ (attachée à $\left.\theta_{0}\right)$ et $(\Gamma, \gamma)$ (attachée à $\left.\theta_{0}^{2}\right)$ comme ci-dessus, les groupes

$$
\Omega(1,1, N / 2)=\left(\begin{array}{ccc}
\Gamma & \mathfrak{J}^{0} & \varpi_{E}^{-1} \mathfrak{H}^{1} \sigma \\
\mathfrak{H}^{1} & \Delta & \mathfrak{J}^{0} \sigma \\
\sigma^{-1} \mathfrak{J}^{0} \varpi_{E} & \sigma^{-1} \mathfrak{H}^{1} & \tau \\
\tau & \Gamma
\end{array}\right) \cap G_{1, N / 2}
$$

et

$$
\Omega(1,2, N / 2)=\left(\begin{array}{ccc}
\Gamma & \mathfrak{J}^{0} & \mathfrak{J}^{0} \sigma \\
\mathfrak{H}^{1} & \Delta & \mathfrak{J}^{0} \sigma \\
\sigma^{-1} \mathfrak{H}^{1} & \sigma^{-1} \mathfrak{H}^{1} & \tau
\end{array}\right) \cap G_{1, N / 2}
$$

portent des paires décomposées au-dessus de $(\iota(\Gamma) \times \Delta, \gamma \otimes \delta)$ relativement à $P_{1, N / 2}$ (noter que $\Delta=\bar{\Gamma}$ ). L'hypothèse (H1) est donc vérifiée. Pour établir (H2) et (H3) on combine le raisonnement de $\mathrm{Bl3}$ §3.3, grandement facilité par l'hypothèse $[E: F]=N$, et des arguments de propagation comme en 1.4. 
Le théorème 1 fournit cette fois des paires couvrantes dans $S p((2 t+1) N, F)$ attachées aux classes d'inertie $\left[G L(N, F)^{\times t} \times S p(N, F), \pi^{\otimes t} \otimes \rho\right]_{S p((2 t+1) N, F)}$. Le théorème 2 donne la description de leur algèbre de Hecke, moyennant les paramètres inconnus du cas $t=1$.

\section{Appendice : Démonstration De La PRoposition 1}

Ce paragraphe est technique et s'appuie fortement sur [BK1] $\$ 7.1$. On y utilise donc les hypothèses et notations 7.1.11, 7.1.13 et 7.2.1 de loc. cit. (Remarquons qu'en niveau 0 la proposition est immédiate: elle revient à remarquer que le radical de l'ordre $\mathfrak{A}$ est $\mathfrak{B}=\left(\begin{array}{ccccc}\mathfrak{P}_{0} & \mathfrak{A}_{0} & \ldots & \mathfrak{A}_{0} \\ \mathfrak{P}_{0} & \mathfrak{P}_{0} & \ddots & \vdots \\ \vdots & \ddots & \ddots & \mathfrak{A}_{0} \\ \mathfrak{P}_{0} & \ldots & \mathfrak{P}_{0} & \mathfrak{P}_{0}\end{array}\right)$.)

On s'intéresse aux sous-anneaux $A^{\prime}$ et $A^{\prime \prime}$ de $A=\operatorname{End}_{F}(V)$ définis par $A^{\prime}=$ $\operatorname{End}_{F}\left(V^{\prime}\right)$ et $A^{\prime \prime}=\operatorname{End}_{F}\left(V^{\prime \prime}\right)$, avec

$$
V^{\prime}=V^{(1)} \oplus \cdots \oplus V^{(t-1)} \quad \text { et } \quad V^{\prime \prime}=V^{(t-1)} \oplus V^{(t)} .
$$

On définit $M^{\prime}=M \cap A^{\prime}, \mathbb{N}^{\prime}=\mathbb{N} \cap A^{\prime}$ et de même pour $M^{\prime \prime}, \mathbb{N}^{\prime \prime}, \mathbb{N}^{\prime-}$ et $\mathbb{N}^{\prime \prime}$. On pose $\mathfrak{A}^{\prime}=\mathfrak{A} \cap A^{\prime}, \mathfrak{A}^{\prime \prime}=\mathfrak{A} \cap A^{\prime \prime}$, et on note $\mathfrak{P}^{\prime}$ et $\mathfrak{P}^{\prime \prime}$ les radicaux respectifs de $\mathfrak{A}^{\prime}$ et $\mathfrak{A}^{\prime \prime}$. On note enfin $B, B^{\prime}, B^{\prime \prime}$ les commutants de $\beta$ dans $A, A^{\prime}, A^{\prime \prime}$ respectivement, et $\mathfrak{B}^{\prime}=B^{\prime} \cap \mathfrak{A}=A^{\prime} \cap \mathfrak{B}$, etc.

Les ordres $\mathfrak{A}^{\prime}$ et $\mathfrak{A}^{\prime \prime}$ sont des ordres héréditaires dans $A^{\prime}$ et $A^{\prime \prime}$, attachés respectivement aux suites de réseaux $\left(L_{k} \cap V^{\prime}\right)_{k \in \mathbb{Z}}$ et $\left(L_{k} \cap V^{\prime \prime}\right)_{k \in \mathbb{Z}}($ [BK1 7.1.6). Il est facile d'écrire ces suites sous forme de chaînes : on a $\mathfrak{A}^{\prime}=\operatorname{End}_{\mathfrak{o}_{F}}^{0}\left(\left\{L_{i}^{\prime}, i \in \mathbb{Z}\right\}\right)$ et $\mathfrak{A}^{\prime \prime}=\operatorname{End}_{\mathfrak{o}_{F}}^{0}\left(\left\{L_{i}^{\prime \prime}, i \in \mathbb{Z}\right\}\right)$, où l'on a défini, pour tout entier $k=\alpha+\beta t$, $0 \leqslant \alpha \leqslant t-1, \beta \in \mathbb{Z}$ :

$$
\begin{aligned}
& L_{\phi(k)}^{\prime}=L_{k} \cap V^{\prime} \quad \text { avec } \quad \phi(k)=\alpha+\beta(t-1)-\left[\frac{(t-1)(k+1)}{t}\right], \\
& L_{\psi(k)}^{\prime \prime}=L_{k} \cap V^{\prime \prime} \quad \text { avec } \quad \psi(\alpha+\beta t)= \begin{cases}2 \beta & \text { si } 0 \leq \alpha \leq t-2, \\
2 \beta+1 & \text { si } \alpha=t-1 .\end{cases}
\end{aligned}
$$

La fonction $\phi$ vérifie : $\phi(k)+\phi(m)-1 \leqslant \phi(k+m) \leqslant \phi(k)+\phi(m)(k, m \in \mathbb{Z})$. Il en découle immédiatement que

$$
\mathfrak{P}^{\phi(m)} \subset \mathfrak{P}^{m} \cap A^{\prime} \subset \mathfrak{P}^{\phi(m)-1} \quad \text { pour tout } m \in \mathbb{Z} .
$$

Le cas de $\psi$ est plus compliqué, mais il nous suffira d'avoir :

Lemme A1. Supposons $t \geqslant 3$. Pour tout entier positif $i$, on a les inclusions suivantes :

si t est impair :

$$
\mathfrak{P}^{\prime\left[\frac{(t-1) i}{2}\right]+1} \subset \mathfrak{P}^{\left[\frac{t i}{2}\right]+1} \quad \text { et } \quad \mathfrak{P}^{\left[\frac{(t-1) i+1}{2}\right]} \cap \mathbb{N}^{\prime} \subset \mathfrak{P}^{\left[\frac{t i+1}{2}\right]},
$$

sit est pair :

$$
\mathfrak{P}^{\prime\left[\frac{(t-1) i+1}{2}\right]} \subset \mathfrak{P}^{\left[\frac{t i+1}{2}\right]} \quad \text { et } \quad \mathfrak{P}^{\prime\left[\frac{(t-1) i}{2}\right]+1} \cap \mathbb{N}^{\prime} \subset \mathfrak{P}^{\left[\frac{t i}{2}\right]+1},
$$

dans les deux cas :

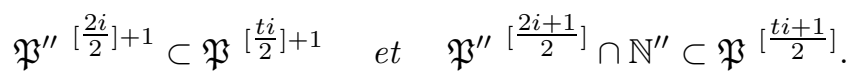


Démonstration. On a dans tous les cas :

$\phi\left(\left[\frac{t i}{2}\right]+1\right) \geqslant\left[\frac{(t-1) i}{2}\right]+1$, avec égalité si $t$ est impair ou si $i$ est pair, et

$\phi\left(\left[\frac{t i+1}{2}\right]\right) \geqslant\left[\frac{(t-1) i+1}{2}\right]$, avec égalité si $t$ est pair ou si $i$ est pair.

Les inclusions de gauche dans (a) et (b) en découlent immédiatement via $(\star)$, de même que les inclusions de droite si $i$ est pair.

Supposons alors $i=2 a+1$ impair ; l'inclusion de droite de (a) équivaut à :

$$
\text { pour tout } x \in \mathfrak{P}^{(t-1) a+\frac{t-1}{2}} \cap \mathbb{N}^{\prime} \text { et tout } k \in \mathbb{Z}: x L_{k} \subset L_{k+t a+\frac{t+1}{2}} .
$$

Ecrivons $k=\alpha+\beta t$ avec $\beta \in \mathbb{Z}$ et $0 \leqslant \alpha \leqslant t-1$. On a $x L_{k}=x L_{\phi(k)}^{\prime}$, donc :

$$
x L_{k} \subset L_{\phi(k)+(t-1) a+\frac{t-1}{2}}^{\prime} .
$$

On a toujours :

$$
\phi(k)+(t-1) a+\frac{t-1}{2} \leqslant \phi\left(k+t a+\frac{t+1}{2}\right) \leqslant \phi(k)+(t-1) a+\frac{t+1}{2}
$$

avec égalité à gauche si $\alpha+\frac{t+1}{2} \geqslant t$, à droite sinon. Dans le premier cas la propriété voulue découle de $(*)$. Dans le second c'est l'hypothèse supplémentaire $x \in \mathbb{N}^{\prime}$ qui va nous permettre de gagner un cran. Soit donc $\alpha<\frac{t-1}{2}$; on a $L_{k}=L_{k+1}+L_{k} \cap V^{(\alpha+1)}$ d'où $x L_{k}=x L_{k+1}+x\left(L_{k} \cap V^{(\alpha+1)}\right)$. On examine séparément les deux termes :

- $\phi\left(k+t a+\frac{t+1}{2}\right)=\phi(k+1)+(t-1) a+\frac{t-1}{2}$ donc $x L_{k+1} \subset L_{k+t a+\frac{t+1}{2}}$.

- $x\left(L_{k} \cap V^{(\alpha+1)}\right)$ est contenu dans $L_{k+a t+\frac{t-1}{2}} \cap\left(V^{(1)} \oplus \cdots \oplus V^{(\alpha)}\right)$, qui coïncide avec $L_{k+a t+\frac{t+1}{2}} \cap\left(V^{(1)} \oplus \cdots \oplus V^{(\alpha)}\right)$ car les deux réseaux ne diffèrent qu'en $V^{\left(\alpha+\frac{t+1}{2}\right)}$, c.q.f.d.

Le raisonnement est semblable pour l'inclusion de droite de (b).

Passons à (c). L'inclusion de gauche s'obtient en vérifiant qu'on a pour tout $i$ : $\psi\left(k+\left[\frac{t i}{2}\right]+1\right) \leqslant \psi(k)+i+1$. Pour l'inclusion de droite, il s'agit de montrer que

$$
\text { pour tout } x \in \mathfrak{P}^{\prime \prime} \cap \mathbb{N}^{\prime \prime} \text { et tout } k \in \mathbb{Z}: x L_{k} \subset L_{k+\left[\frac{t i+1}{2}\right]} .
$$

Comme $x L_{k}=x L_{\psi(k)}^{\prime \prime} \subset L_{\psi(k)+i}^{\prime \prime}$, la propriété voulue est certainement vraie pour tout $k$ tel que $\psi(k)+i \geqslant \psi\left(k+\left[\frac{t i+1}{2}\right]\right)$. Cette dernière (in-)égalité est valide pour tout $k$ lorsque $i$ est pair. Si $i=2 a+1$ est impair, on écrit $k=\alpha+\beta t$ avec $\beta \in \mathbb{Z}$ et $0 \leqslant \alpha \leqslant t-1$; l'inégalité est valide si $\alpha=t-1$ ou $\alpha<\left[\frac{t}{2}\right]$. Ne reste à examiner que les cas $\left[\frac{t}{2}\right] \leqslant \alpha \leqslant t-2: \psi(k)$ est alors pair, et nous pouvons enfin faire intervenir l'hypothèse $x \in \mathbb{N}^{\prime \prime}$. En effet :

$$
x L_{k}=x L_{\psi(k)}^{\prime \prime} \subset L_{\psi(k)+2 a+1}^{\prime \prime} \cap V^{(t-1)}=L_{\psi(k)+2 a+2}^{\prime \prime} \cap V^{(t-1)}
$$

car les deux réseaux ne diffèrent qu'en $V^{(t)}$. Il suffit donc dans ce dernier cas de vérifier l'inégalité $\psi(k)+i+1 \geqslant \psi\left(k+\left[\frac{t i+1}{2}\right]\right)$, ce qui est aisé. 
Lemme A2. Supposons $t \geqslant 3$. On a les inclusions suivantes:

sit est impair :

$$
\mathfrak{H}^{1}\left(\beta, \mathfrak{A}^{\prime}\right) \subset \mathfrak{H}^{1}(\beta, \mathfrak{A}) \quad \text { et } \quad \mathfrak{J}^{1}\left(\beta, \mathfrak{A}^{\prime}\right) \cap \mathbb{N}^{\prime} \subset \mathfrak{J}^{1}(\beta, \mathfrak{A}),
$$

si t est pair :

$$
\mathfrak{J}^{1}\left(\beta, \mathfrak{A}^{\prime}\right) \subset \mathfrak{J}^{1}(\beta, \mathfrak{A}) \quad \text { et } \quad \mathfrak{H}^{1}\left(\beta, \mathfrak{A}^{\prime}\right) \cap \mathbb{N}^{\prime} \subset \mathfrak{H}^{1}(\beta, \mathfrak{A}),
$$

dans les deux cas:

$$
\mathfrak{H}^{1}\left(\beta, \mathfrak{A}^{\prime \prime}\right) \subset \mathfrak{H}^{1}(\beta, \mathfrak{A}) \quad \text { et } \quad \mathfrak{J}^{1}\left(\beta, \mathfrak{A}^{\prime \prime}\right) \cap \mathbb{N}^{\prime \prime} \subset \mathfrak{J}^{1}(\beta, \mathfrak{A}) .
$$

Démonstration. Par définition, on a $\mathfrak{H}^{1}(\beta, \mathfrak{A})=\mathfrak{H}(\beta, \mathfrak{A}) \cap \mathfrak{P}$ et des égalités analogues dans $\mathfrak{A}^{\prime}$ et $\mathfrak{A}^{\prime \prime}$. Comme $\mathfrak{P}^{\prime}$ et $\mathfrak{P}^{\prime \prime}$ sont contenus dans $\mathfrak{P}$ il suffit d'établir les inclusions annoncées en remplaçant partout $\mathfrak{H}^{1}$ par $\mathfrak{H}$.

Rappelons que la strate sous-jacente à tout ceci est la strate simple $\left[\mathfrak{A}, t n_{0}, 0, \beta\right]$ et commençons par le cas où $\beta$ est minimal. Alors $\mathfrak{H}(\beta, \mathfrak{A})=\mathfrak{B}+\mathfrak{P}^{\left[\frac{t n_{0}}{2}\right]+1}$, $\mathfrak{H}\left(\beta, \mathfrak{A}^{\prime}\right)=\mathfrak{B}^{\prime}+\mathfrak{P}^{\prime}\left[\frac{(t-1) n_{0}}{2}\right]+1$ et $\mathfrak{H}\left(\beta, \mathfrak{A}^{\prime \prime}\right)=\mathfrak{B}^{\prime \prime}+\mathfrak{P}^{\prime \prime}\left[\frac{2 n_{0}}{2}\right]+1$. Mais $\mathfrak{B}^{\prime}$ et $\mathfrak{B}^{\prime \prime}$ sont contenus dans $\mathfrak{B}$ donc les inclusions voulues sont des conséquences immédiates du lemme A1.

Passons maintenant à un élément $\beta$ quelconque. On raisonne par "induction le long de $\beta$ " comme dans BK1, 7.1.12 : on choisit $\gamma$ comme dans loc. cit. et on suppose les assertions vraies pour $\gamma$. En posant $r=-k_{0}\left(\beta, \mathfrak{A}_{0}\right)$, on a par définition :

$$
\begin{aligned}
& \mathfrak{H}\left(\beta, \mathfrak{A}^{\prime}\right)=\mathfrak{B}^{\prime}+\mathfrak{H}\left(\gamma, \mathfrak{A}^{\prime}\right) \cap \mathfrak{P}^{\prime}\left[\frac{(t-1) r}{2}\right]+1, \\
& \mathfrak{H}\left(\beta, \mathfrak{A}^{\prime \prime}\right)=\mathfrak{B}^{\prime \prime}+\mathfrak{H}\left(\gamma, \mathfrak{A}^{\prime \prime}\right) \cap \mathfrak{P}^{\prime \prime}\left[\frac{2 r}{2}\right]+1 \\
& \mathfrak{H}(\beta, \mathfrak{A})=\mathfrak{B}+\mathfrak{H}(\gamma, \mathfrak{A}) \cap \mathfrak{P}\left[\frac{t r}{2}\right]+1
\end{aligned}
$$

Les inclusions voulues découlent donc de l'hypothèse sur $\gamma$ et du lemme A1.

La démonstration est la même pour $\mathfrak{J}$, en remplaçant partout les expressions $\left[\frac{i n_{0}}{2}\right]+1$ par des $\left[\frac{i n_{0}+1}{2}\right]$.

Proposition A1. Supposons $t \geqslant 3$. On a les égalités suivantes :

si t est impair :

(a) $\quad \mathfrak{H}^{1}\left(\beta, \mathfrak{A}^{\prime}\right) \cap \mathbb{N}^{\prime-}=\mathfrak{H}^{1}(\beta, \mathfrak{A}) \cap \mathbb{N}^{\prime-} \quad$ et $\quad \mathfrak{J}^{1}\left(\beta, \mathfrak{A}^{\prime}\right) \cap \mathbb{N}^{\prime}=\mathfrak{J}^{1}(\beta, \mathfrak{A}) \cap \mathbb{N}^{\prime}$, si t est pair :

(b) $\quad \mathfrak{J}^{1}\left(\beta, \mathfrak{A}^{\prime}\right) \cap \mathbb{N}^{\prime-}=\mathfrak{J}^{1}(\beta, \mathfrak{A}) \cap \mathbb{N}^{\prime-} \quad$ et $\quad \mathfrak{H}^{1}\left(\beta, \mathfrak{A}^{\prime}\right) \cap \mathbb{N}^{\prime}=\mathfrak{H}^{1}(\beta, \mathfrak{A}) \cap \mathbb{N}^{\prime}$, dans les deux cas :

(c) $\quad \mathfrak{H}^{1}\left(\beta, \mathfrak{A}^{\prime \prime}\right) \cap \mathbb{N}^{\prime \prime}=\mathfrak{H}^{1}(\beta, \mathfrak{A}) \cap \mathbb{N}^{\prime \prime}$ et $\mathfrak{J}^{1}\left(\beta, \mathfrak{A}^{\prime \prime}\right) \cap \mathbb{N}^{\prime \prime}=\mathfrak{J}^{1}(\beta, \mathfrak{A}) \cap \mathbb{N}^{\prime \prime}$

Démonstration. Le lemme A2 donne dans chaque cas l'inclusion du membre de gauche dans le membre de droite. L'égalité va résulter d'un argument de maximalité des paires couvrantes que l'on ne détaille que dans le cas (b), $t$ pair.

Les résultats de BK1] (7.3.2, 7.2.17 et 7.1.17) affirment que le produit

$$
\left(H^{1}(\beta, \mathfrak{A}) \cap(1+\mathbb{N})\right) \cdot(J(\beta, \mathfrak{A}) \cap M) \cdot\left(J^{1}(\beta, \mathfrak{A}) \cap\left(1+\mathbb{N}^{-}\right)\right)
$$


porte une paire couvrante de $\left(\Gamma^{\times t}, \gamma^{\otimes t}\right)$ relativement à $M(1+\mathbb{N})$. Par transitivité (voir 1.1) on en déduit que le produit

$$
\left(H^{1}(\beta, \mathfrak{A}) \cap\left(1+\mathbb{N}^{\prime}\right)\right) \cdot\left(J(\beta, \mathfrak{A}) \cap M^{\prime}\right) \cdot\left(J^{1}(\beta, \mathfrak{A}) \cap\left(1+\mathbb{N}^{\prime-}\right)\right)
$$

porte une paire décomposée au-dessus de $\left(\Gamma^{\times t-1}, \gamma^{\otimes t-1}\right)$ relativement à $M^{\prime}\left(1+\mathbb{N}^{\prime}\right)$. Or ce produit contient terme à terme, par le lemme A2, le produit "d'origine"

$$
\left(H^{1}\left(\beta, \mathfrak{A}^{\prime}\right) \cap\left(1+\mathbb{N}^{\prime}\right)\right) \cdot\left(J\left(\beta, \mathfrak{A}^{\prime}\right) \cap M^{\prime}\right) \cdot\left(J^{1}\left(\beta, \mathfrak{A}^{\prime}\right) \cap\left(1+\mathbb{N}^{\prime}\right)\right)
$$

qui porte lui-même une paire couvrante de $\left(\Gamma^{\times t-1}, \gamma^{\otimes t-1}\right)$ relativement à $M^{\prime}\left(1+\mathbb{N}^{\prime}\right)$.

Comme une paire couvrante est en particulier une paire décomposée maximale (B12] Théorème 1), on doit avoir égalité terme à terme des facteurs.

Démonstration de la proposition 1. On la prouve par récurrence sur $t$, en utilisant la décomposition $V=\bigoplus_{j=1}^{t} V^{(j)}$ et l'ordre $\mathfrak{A}$ du paragraphe 1.2. Le cas $t=1$ est tautologique et le cas $t=2$ est traité dans B13, Lemme 2.2. Supposons donc la proposition démontrée à l'ordre $t-1$ avec $t \geqslant 3$. On sait déjà (BK1, 7.1.12) que les blocs diagonaux de $\mathfrak{H}^{1}(\beta, \mathfrak{A})$ et $\mathfrak{J}^{1}(\beta, \mathfrak{A})$ sont égaux à $\mathfrak{H}^{1}$ et $\mathfrak{J}^{1}$ respectivement. Pour le reste, les deux ingrédients de la preuve sont la proposition A1 bien sûr, et le corollaire 3.1.11 de [BK1], qui assure que $\mathfrak{H}^{1}(\beta, \mathfrak{A})$ et $\mathfrak{J}^{1}(\beta, \mathfrak{A})$ sont normalisés par

$$
\Pi=\left(\begin{array}{ccccc}
0 & I_{N} & 0 & \ldots & 0 \\
\vdots & \ddots & \ddots & \ddots & \vdots \\
\vdots & & \ddots & \ddots & 0 \\
0 & & & \ddots & I_{N} \\
\varpi_{E} & 0 & \ldots & \ldots & 0
\end{array}\right) .
$$

Si $t$ est pair, on a $\mathfrak{H}^{1}(\beta, \mathfrak{A}) \cap \mathbb{N}^{\prime}=\mathfrak{H}^{1}\left(\beta, \mathfrak{A}^{\prime}\right) \cap \mathbb{N}^{\prime}$ par la proposition A1. Cela permet, grâce à l'hypothèse de récurrence, de déterminer les blocs $(i, j)$ avec $i>j>1$ de $\mathfrak{H}^{1}(\beta, \mathfrak{A})$. La normalisation par $\Pi$ fournit ensuite les blocs $(i, j)$ manquants, à l'exception de ceux pour lesquels $j=i+1$ (modulo $t$ ). Il reste à déterminer un des blocs de cette orbite manquante sous $\Pi$ : c'est la proposition A1, (c) qui nous fournit le bloc $(1,2)$.

La détermination de $\mathfrak{J}^{1}(\beta, \mathfrak{A})$ se fait de manière semblable, ainsi que le cas où $t$ est impair (pour lequel on peut facilement montrer a priori que $\mathfrak{H}^{1}(\beta, \mathfrak{A})$ et $\mathfrak{J}^{1}(\beta, \mathfrak{A})$ ne diffèrent que par leurs blocs diagonaux).

\section{REFERENCES}

[BB] L. Blasco et C. Blondel, Algèbres de Hecke et séries principales généralisées de $S p_{4}(F)$, Proc. London Math. Soc. (3) 85 (2002), 659-685. MR.1936816 (2003k:22025)

[B11] C. Blondel, Critère d'injectivité pour l'application de Jacquet, C. R. Acad. Sci. Paris (325) I (1997), 1149-1152. MR.1490115 (98k:22069)

[B12] C. Blondel, Quelques propriétés des paires couvrantes, Math. Annalen (2) 331 (2005), 243-257. MR2115455 (2005k:20117)

[B13] C. Blondel, $S p(2 N)$-covers for self-contragredient supercuspidal representations of $G L(N)$, Ann. scient. Ec. Norm. Sup. 37 (2004), 533-558. MR2097892(2006a:22013)

[Bo] N. Bourbaki, Groupes et algèbres de Lie, Chapitres 4, 5 et 6, (Masson, 1981). MR0647314 (83g:17001)

[Bu] C. J. Bushnell, Hereditary orders, Gauss sums and supercuspidal representations of $G L_{N}$, J. Reine Angew. Math. 375/376 (1987), 184-210. MR0882297 (88e:22024)

[BK1] C. J. Bushnell and P. C. Kutzko, The admissible dual of $G L_{n}$ via compact open subgroups, Annals of Math. Studies 129 (Princeton University Press, 1993). MR.1204652 (94h:22007) 
[BK2] C.J. Bushnell and P.C. Kutzko, Smooth representations of reductive $p$-adic groups: structure theory via types, Proc. London Math. Soc. 77 (1998), 582-634. MR1643417 (2000c:22014)

[BK3] C.J. Bushnell and P.C. Kutzko, Types in reductive $p$-adic groups: the Hecke algebra of a cover, Proc. Amer. Math. Soc. (2) 129 (2001), 601-607. MR1712937(2001j:22023)

[Go] D. Goldberg, Reducibility of induced representations for $S p(2 N)$ and $S O(N)$, Amer. J. of Math. 116 (1994), 1101-1151. MR.1296726 (95g:22016)

[Mo] L. Morris, Tamely ramified intertwining algebras, Invent. Math. 114 (1993), 1-54. MR.1235019 (94g:22035)

[ST] P. J. Sally Jr. and M. Tadić, Induced representations and classifications for $G S p(2, F)$ and Sp $(2, F)$, Mémoire SMF 52, supplément au Bull. Soc. Math. de France 121 (1993), 75-133. MR.1212952 (94e:22030)

[St] S. Stevens, Intertwining and supercuspidal types for $p$-adic classical groups, Proc. London Math. Soc. 83 (2001), 120-140. MR.1829562 (2002b:22031)

[Ta] M. Tadić, Square integrable representations of classical $p$-adic groups corresponding to segments, Represent. Theory 3 (1999), 58-89. MR1698200 (2000d:22020)

[Wa] J.-L. Waldspurger, Algèbres de Hecke et induites de représentations cuspidales, pour GL(N), J. Reine Angew. Math. 370 (1986), 127-191. MR0852514 (87m:22048)

C.N.R.S. - Théorie des Groupes-Case 7012, Institut de Mathématiques de Jussieu, Université PARIS 7, F-75251 PARIS Cedex 05.

E-mail address: blondel@math.jussieu.fr 